\title{
Biodiversidad de esponjas en arrecifes rocosos del Chocó norte, Pacífico colombiano
}

\section{Biodiversity of sponges in rocky reefs of North Chocó, Colombian Pacific}

\author{
Nicoll Lizarazo ${ }^{1,3}$, Sven Zea ${ }^{2 *}$, Luis Chasqui ${ }^{3}$ y Natalia Rincón-Díaz ${ }^{3}$ \\ (iD) $0000-0001-6163-7185$ (iD 0000-0002-5657-4877 (iD 0000-0002-9381-2301 (iD $0000-0002-4908-4802$
}

\begin{abstract}
1. Programa de Biología Marina, Facultad de Ciencias Naturales e Ingeniería, Universidad de Bogotá Jorge Tadeo Lozano, sede Caribe, Santa Marta, Colombia.nicollp.lizarazor@utadeo.edu.co

2. Instituto de Estudios en Ciencias del Mar (Cecimar), Universidad Nacional de Colombia, sede Caribe, Santa Marta, Colombia. sezeas@unal.edu.co*

3. Instituto de Investigaciones Marinas y Costeras “José Benito Vives de Andréis” (Invemar), Santa Marta D.T.H.C., Colombia.luis.chasqui@invemar. org.co,,natalia.rincon@invemar.org.co

* Autor de correspondencia.
\end{abstract}

\section{RESUMEN}

$\mathrm{E}$ n comparación con otros grupos de invertebrados, la taxonomía de esponjas (Porífera) ha sido poco desarrollada en el Pacífico Oriental Tropical (POT), en general, y en el Pacífico colombiano, en particular. Este trabajo busca incrementar el conocimiento de la biodiversidad del grupo en los arrecifes rocosos del norte del Chocó. Se recolectaron muestras de esponjas mediante buceo autónomo, las cuales fueron procesadas e identificadas en laboratorio por métodos estándar. Se confirmó la baja riqueza de especies y la predominancia de morfologías incrustantes y hábitos crípticos, que ha sido encontrada en otros estudios en la región. Se identificaron 24 morfotipos pertenecientes a 13 órdenes, 17 familias y 21 géneros. Doce (12) morfotipos se reconocieron a nivel de género o con identidad específica tentativa y los otros 12 fueron confirmados a nivel de especie. Seis (6) especies son morfológicamente similares a las del Caribe, lo que indica que las poblaciones posiblemente no han divergido lo suficiente desde el cierre del istmo de Panamá o, alternativamente, pueden haber cruzado recientemente el canal de Panamá.

PALABRAS CLAVE: biodiversidad de esponjas, Demospongiae, arrecifes rocosos del Pacífico, Pacífico colombiano, Pacífico Oriental Tropical.

\section{ABSTRACT}

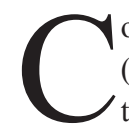

ompared to other groups of invertebrates, sponge (Porifera) taxonomy has been poorly developed in the Eastern Tropical Pacific (ETP) in general and in the Colombian Pacific in particular. This work aims to increase knowledge of the group's biodiversity in the rocky reefs of northern Chocó. Sponge samples were collected by autonomous diving, which were then processed and identified in the laboratory by standard methods. It was confirmed the low species richness and the predominance of encrusting morphologies and cryptic habits, which have been found in other studies in the region. Twenty-four (24) morphotypes belonging to 13 orders, 17 families and 21 genera were identified. Twelve (12) morphotypes were recognized at the genus level or with a tentative specific identity and the other 12 were confirmed at the species level. Six (6) species are morphologically similar to those of the Caribbean, indicating that populations may not have diverged sufficiently since the closure of the Isthmus of Panama or, alternatively, may have recently crossed the Panama Canal.

KEYWORDS: sponges' biodiversity, Demospongiae, Pacific rocky reefs, Colombian Pacific, Eastern Tropical Pacific.

DOI: https://doi.org/10.25268/bimc.invemar.2020.49.2.889 Publicado por INVEMAR

Este es un manuscrito de acceso abierto bajo la licencia CC 


\section{INTRODUCCIÓN}

En la costa norte del departamento del Chocó (Pacífico colombiano), se destacan formaciones rocosas conocidas como riscales y morros, que son arrecifes rocosos sumergidos y pequeños islotes, respectivamente (Guzmán et al., 2004). Estas formaciones compuestas por rocas ígneas basálticas máficas y ultramáficas tienen diferentes niveles de complejidad topográfica, moldeada por siglos de exposición a factores de erosión marina que han creado paredes rocosas y cúmulos de grandes rocas con grietas y cuevas de tamaño variable. Los riscales y morros proveen sustrato duro para el asentamiento de biota bentónica como corales blandos, algas y esponjas, así como refugio a una amplia variedad de invertebrados móviles y peces (Velandia y Díaz, 2016).

Entre la biota característica de esas formaciones rocosas, las esponjas (Porifera) están entre los más conspicuos e importantes organismos bentónicos sésiles. Son filtradores que incorporan al fondo materia orgánica particulada y disuelta de la columna de agua que luego, por medio de un proceso de regeneración celular, devuelven en forma de nutrientes inorgánicos y detritus particulado al medio (Goeij et al., 2008, 2013). En el Caribe y en mares del océano Índico Occidental e Indonesia, las esponjas son los organismos más conspicuos y diversos en las aguas costeras, después de los corales y las algas, ya que pueden encontrarse en diferentes hábitats como raíces de manglar, arrecifes de coral y fondos blandos, entre otros (Zea, 1987, 1998; Van Soest, 1994; Hooper et al., 2002). En comparación con otros grupos taxonómicos de invertebrados, el estudio sistemático de las esponjas está poco desarrollado a raíz de la escasez de taxónomos especializados, la dificultad para identificar las especies, la ambigüedad de las características y lo disperso de la literatura (Hooper et al., 2002). No obstante, la popularización del buceo autónomo, el desarrollo de la fotografía submarina y la implementación de técnicas histológicas y moleculares han ayudado a paliar el problema (Worheide et al., 2005; Cárdenas et al., 2012).

Aunque esto ha permitido aumentar el inventario de la biodiversidad del grupo en las últimas décadas, se cree que el número de especies válidas descritas corresponde, como mucho, a la mitad de las especies existentes (Van Soest et al., 2012). Es importante entonces continuar realizando esfuerzos para consolidar el inventario de la biodiversidad global de poríferos, especialmente en regiones menos exploradas como el Pacífico Oriental y, en particular, en la costa Pacífica de Colombia.

\section{INTRODUCTION}

On the north coast of the Chocó department (Colombian Pacific), there are rock formations known as riscales and morros, which are submerged rocky reefs and small islets or cays, respectively (Guzmán et al., 2004). These formations are composed of mafic and ultramafic basaltic igneous rocks having different levels of topographic complexity, shaped by centuries of exposure to marine erosion factors that have created rock faces and large rock clusters with cracks and caves of varying size. The cliffs and hills provide hard substratum for the settlement of benthic biotas such as soft corals, algae, and sponges, as well as shelter for a wide variety of mobile invertebrates and fish (Velandia and Díaz, 2016).

Among the characteristic biota of these rock formations, sponges (Porifera) are among the most conspicuous and important sessile benthic organisms. They are filters feeders that incorporate particulate and dissolved organic matter from the water column to the bottom, which then, through a process of cellular regeneration, return in the form of inorganic nutrients and particulate detritus to the environment (Goeij et al., 2008, 2013). In the Caribbean and the seas of the Western Indian Ocean and Indonesia, sponges are the most conspicuous and diverse organisms in coastal waters, after corals and algae, as they can be found in different habitats such as mangrove roots, coral reefs, and soft bottoms, among others (Zea, 1987, 1998; Van Soest, 1994; Hooper et al., 2002). In comparison with other taxonomic groups of invertebrates, the systematic study of sponges is underdeveloped due to the scarcity of specialized taxonomists, the difficulty of identifying the species, the ambiguity of the characters, and the dispersion of the literature (Hooper et al., 2002). However, the popularization of autonomous diving, the development of underwater photography, and the implementation of histological and molecular techniques have helped in alleviating the problem (Worheide et al., 2005; Cárdenas et al., 2012).

Although this has made it possible to increase the group's biodiversity inventory in recent decades, it is believed that the number of valid species described corresponds, at most, to half of the existing species (Van Soest et al., 2012). It is therefore important to continue making efforts to consolidate the inventory of global Poriferan biodiversity, especially in less explored regions such as the Eastern Pacific and, in particular, on the Pacific coast of Colombia. 
Las primeras descripciones de esponjas en la costa Pacífica americana se realizaron a partir de material obtenido desde buques de investigación como la expedición Challenger, donde Ridley y Dendy (1887) describieron 23 especies de esponjas monoaxónicas para el sur de Chile y la Patagonia. Posteriormente, Vosmaer y Vernhout (1902) estudiaron seis especies del género Placospongia recolectadas en América Central y del Sur por la expedición Siboga, y von Lendenfeld (1910) describió 46 especies de la familia Geodidae recolectadas por el barco Albatross. En el siglo XX se comenzaron a desarrollar estudios locales más exhaustivos. De Laubenfels (1932) describió 101 especies marinas y de agua dulce de California y De Laubenfels (1935) añadió ocho nuevas especies de aguas someras de Baja California, México. Green y Gómez (1986) registraron 14 especies de esponjas en la bahía de Mazatlán (Sinaloa, México), siendo ese el primer trabajo para la costa rocosa del Pacífico tropical. Desqueyroux-Faúndez y Van Soest (1997) describieron 45 especies de esponjas de aguas poco profundas en las islas Galápagos, Van Soest y Hajdu (2000) describieron seis especies de esponjas de la costa Pacífica de Panamá y Díaz et al. (2005) describieron una nueva especie de esponja de aguas someras en el golfo de Chiriquí en Panamá.

Recientemente, los trabajos se han concentrado en la costa Pacífica mexicana. Por ejemplo, Carballo et al. (2004) elaboraron un completo estudio de las esponjas excavadoras de coral, y Carballo y Cruz-Barraza (2008) registraron una nueva especie para el Parque Nacional Isla Isabel en el mar de Cortés. Luego, Vega (2012) registró 88 especies de esponjas en el Pacífico mexicano, de las cuales 18 fueron nuevos registros y posiblemente 33 son especies nuevas para la ciencia. El más reciente trabajo es el de Carballo et al. (2019), que incluye 87 especies de esponjas asociadas a arrecifes de coral a lo largo del Pacífico mexicano.

Por otra parte, algunos trabajos han abordado la cuestión de la similitud en las especies de esponjas a ambos lados del istmo de Panamá, un tema de gran interés en el contexto evolutivo de la fauna marina del Caribe y del Pacífico Oriental Tropical (POT). Por ejemplo, De Laubenfels (1936b) efectuó una comparación entre esponjas de aguas poco profundas en el lado Pacífico del canal de Panamá con las del lado Caribe y encontró características morfológicas diferenciables, trabajo corroborado posteriormente por Wulff (1996).

Para el Pacífico colombiano solo se conocen tres trabajos no publicados que, en suma, han registrado 33 especies de esponjas (Narváez, 1999; Escobar, 2000; García-
The first descriptions of sponges on the American Pacific coast were made from the material obtained from research vessels such as the Challenger expedition, where Ridley and Dendy (1887) described 23 species of monoaxonic sponges for southern Chile and Patagonia. Subsequently, Vosmaer and Vernhout (1902) studied six species of the genus Placospongia collected in Central and South America by the Siboga expedition, and von Lendenfeld (1910) described 46 species of the Geodidae family collected by the ship Albatross. In the 20th century, more exhaustive local studies were developed. De Laubenfels (1932) described 101 freshwater and marine species from California and De Laubenfels (1935) added eight new shallow-water species from Baja California, Mexico. Green and Gómez (1986) recorded 14 species of sponges in the Bay of Mazatlán (Sinaloa, Mexico), this being the first work for the rocky coast of the tropical Pacific. Desqueyroux-Faúndez and Van Soest (1997) described 45 species of sponges from shallow waters in the Galapagos Islands, Van Soest and Hajdu (2000) described six species of sponges from the Pacific coast of Panama, and Díaz et al. (2005) described a new species of shallowwater sponge in the Gulf of Chiriquí in Panama.

Recently, work has focused on the Mexican Pacific coast. For example, Carballo et al. (2004) carried out a complete study of coral burrowing sponges, and Carballo and Cruz-Barraza (2008) recorded a new species for Isla Isabel National Park in the Sea of Cortez. Later, Vega (2012) recorded 88 species of sponges in the Mexican Pacific, of which 18 were new records and possibly 33 are species new to science. The most recent work is that of Carballo et al. (2019), which includes 87 species of sponges associated with coral reefs throughout the Mexican Pacific.

On the other hand, some studies have addressed the question of the similarity in the sponge species on both sides of the Isthmus of Panama, a topic of great interest in the evolutionary context of the marine fauna of the Caribbean and the Eastern Tropical Pacific (ETP). For example, De Laubenfels (1936b) made a comparison between shallow-water sponges on the Pacific side of the Panama Canal with those on the Caribbean side and found differentiable morphological characteristics, a work subsequently corroborated by Wulff (1996).

For the Colombian Pacific, only three unpublished works are known that, in sum, have recorded 33 species of sponges (Narváez, 1999; Escobar, 2000; García-Suárez et al., 2012). These studies have focused on the southern 
Suárez et al., 2012). Esos trabajos se han enfocado en la porción sur del Pacífico colombiano y, principalmente, en esponjas asociadas a ecosistemas coralinos. Considerando estos antecedentes, el presente estudio busca contribuir al conocimiento de la biodiversidad de esponjas del Pacífico colombiano y del POT en general, con énfasis en las esponjas asociadas a los arrecifes rocosos de la porción norte del Chocó.

\section{ÁREA DE ESTUDIO}

El estudio se realizó en el Pacífico norte colombiano, entre cabo Corrientes $\left(5^{\circ} 29^{\prime} \mathrm{N}, 77^{\circ} 32^{\prime} \mathrm{W}\right)$ y cabo Marzo $\left(6^{\circ} 49^{\prime} \mathrm{N}, 77^{\circ} 41^{\prime} \mathrm{W}\right)$. En esta área, el fondo marino se caracteriza por una plataforma continental de extensión variable, estrecha y empinada frente a los cabos y ancha y tendida en los golfos. Esta topografía submarina es el resultado de la interacción entre una abundante escorrentía terrestre y las fuerzas erosivas marinas que han actuado por siglos en antiguas costas rocosas de origen ígneo, asociadas a la formación de la serranía del Baudó (Posada et al., 2009).

En ese fondo marino más bien quebrado hay docenas de arrecifes rocosos, conocidos localmente como "morros" y "riscales", los cuales surgen de profundidades variables y a diferentes distancias de la costa. En esas formaciones rocosas, compuestas de rocas ígneas basálticas máficas y ultramáficas, se observan diferentes niveles de complejidad topográfica; pero, en su mayoría, consisten en paredes rocosas y grupos de grandes rocas con cuevas y agujeros de tamaño variable. Estas estructuras sumergidas ofrecen un sustrato duro ideal para el asentamiento de fauna y flora bentónicas ( $v . g$. corales blandos, esponjas, algas erectas e incrustantes, tunicados y briozoos, entre otros), así como refugio para centenares de invertebrados vágiles y peces, lo que hace de estos sitios importantes caladeros de pesca artesanal (Velandia y Díaz, 2016). El esfuerzo de muestreo se concentró en los arrecifes rocosos someros de los sectores de cabo Corrientes, golfo de Tribugá, bahía Solano, bahía Cupica, Punta Cruces y cabo Marzo (Figura 1).

\section{MATERIALES Y MÉTODOS}

Durante una expedición realizada en 2015 se evaluaron 14 estaciones de muestreo entre 11-19 m de profundidad, donde se fotografiaron todas las esponjas presentes y se recolectaron especímenes completos o fragmentos que se fijaron en etanol al $96 \%$. En el laboratorio se realizaron montajes permanentes de espículas limpias portion of the Colombian Pacific and, mainly, on sponges associated with coral ecosystems. Considering these antecedents, this study seeks to contribute to the knowledge of the biodiversity of sponges of the Colombian Pacific and the ETP in general, with emphasis on the sponges associated with the rocky reefs of the northern portion of the Chocó.

\section{STUDY AREA}

The study was carried out in Colombian North Pacific, between Cape Corrientes $\left(5^{\circ} 29^{\prime} \mathrm{N}, 77^{\circ} 32^{\prime} \mathrm{W}\right)$ and Cape Marzo $\left(6^{\circ} 49^{\prime} \mathrm{N}, 77^{\circ} 41^{\prime} \mathrm{W}\right)$. In this area, the seabed is characterized by a continental shelf of variable extension, narrow and steep in front of the headlands and wide and lying in the gulfs. This underwater topography is the result of the interaction between an abundant land runoff and marine erosive forces that have acted for centuries on ancient rocky coasts of igneous origin, associated with the formation of the Baudo mountain range (Posada et al., 2009).

In this rather broken seabed there are dozens of rocky reefs, known locally as "morros" and "riscales", which arise from variable depths and at different distances from the coast. In these rock formations, composed of mafic and ultramafic igneous basalt rocks, different levels of topographic complexity are observed; but, for the most part, they consist of rocky walls and groups of large rocks with caves and holes of varying size. These submerged structures offer an ideal hard substratum for the settlement of benthic fauna and flora (e.g. soft corals, sponges, erect and encrusting algae, tunicates and bryozoans, among others), as well as shelter for hundreds of fragile invertebrates and fish, which makes these important artisanal fishing grounds (Velandia and Díaz, 2016). The sampling effort was concentrated on the shallow rocky reefs in the sectors of Cape Corrientes, Gulf of Tribugá, Solano Bay, Cupica Bay, Punta Cruces and Cape Marzo (Figure 1).

\section{MATERIALS AND METHODS}

During an expedition carried out in 2015, 14 sampling stations between 11-19 $\mathrm{m}$ depth were evaluated, where all the sponges present were photographed and complete specimens or fragments were collected that were fixed in $96 \%$ ethanol. Permanent mounts of clean spicules and skeletons were made in the laboratory, as described in Zea (1987), which were observed and photographed using an optical microscope (Zeiss, Axio Lab. A1). Up to 


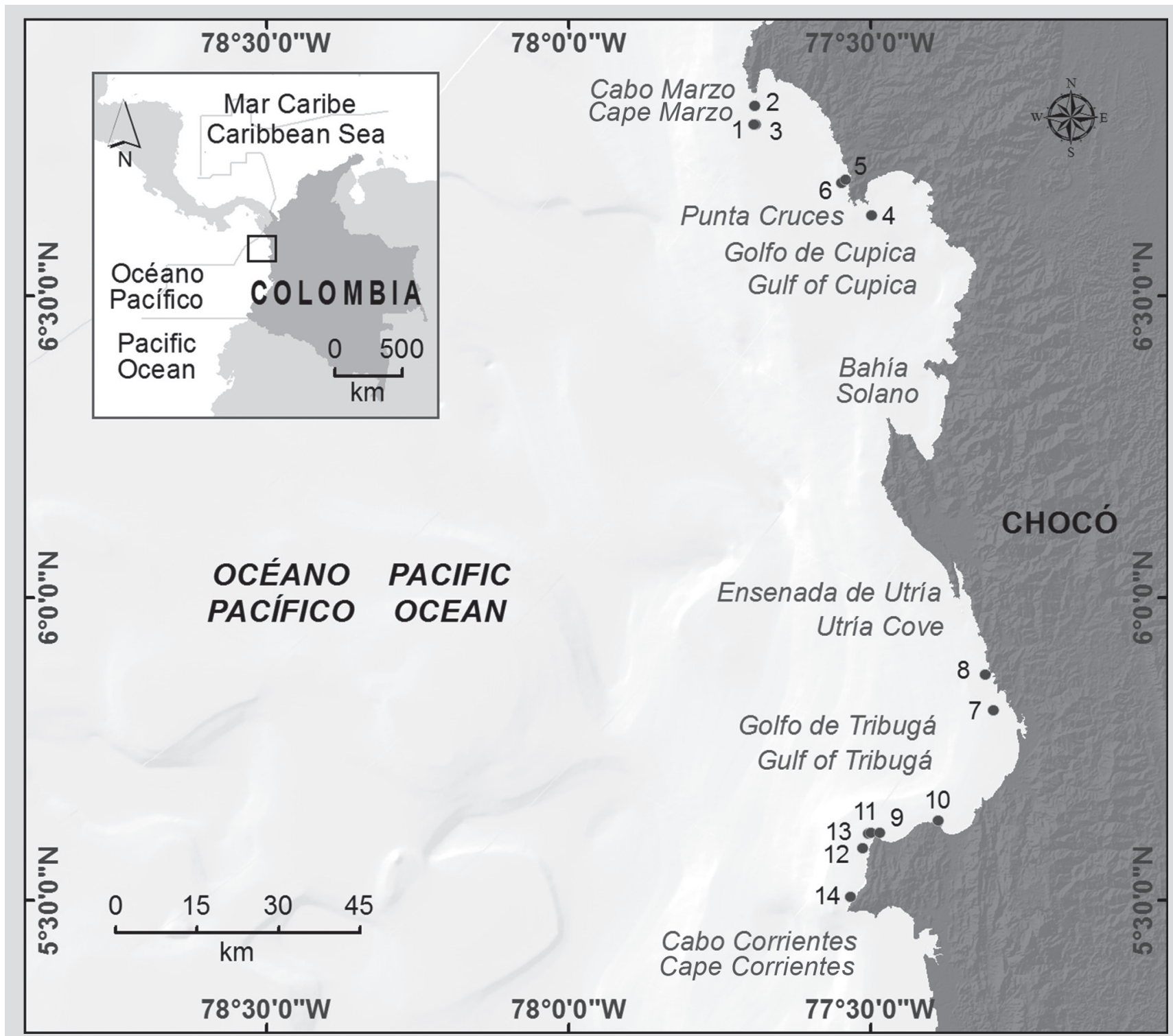

Figura 1. Área de estudio en el sector norte del departamento del Chocó (Pacífico colombiano). Las localidades de muestreo están numeradas: 1) Piedra de Rodrigo (6 $47^{\prime} 2,07^{\prime \prime}$ N, $77^{\circ} 41^{\prime} 36,6^{\prime \prime}$ W). 2) Piedra de Eroito (6 $\left.\left.6^{\circ} 48^{\prime} 52,84^{\prime \prime} \mathrm{N}, 77^{\circ} 41^{\prime} 32,99^{\prime \prime} \mathrm{W}\right) .3\right)$ La Foca $\left(6^{\circ} 40^{\prime} 42,7044^{\prime \prime} \mathrm{N}\right.$, $77^{\circ} 41^{\prime} 42,71^{\prime \prime}$ W). 4) La Viuda (6 $37^{\prime} 58,872^{\prime \prime}$ N, $\left.77^{\circ} 29^{\prime} 58,92^{\prime \prime} \mathrm{W}\right)$. 5) La Mina $\left(6^{\circ} 41^{\prime} 11,76^{\prime \prime} \mathrm{N}, 77^{\circ} 32^{\prime} 57,48^{\prime \prime} \mathrm{W}\right)$. 6) La PargueraPiñas $\left(6^{\circ} 41^{\prime} 30,084^{\prime \prime} N, 77^{\circ} 32^{\prime} 29,76^{\prime \prime}\right.$ W). 7) Morros de Jurubidá ( $\left.\left.5^{\circ} 48^{\prime} 47,16^{\prime \prime} \mathrm{N}, 77^{\circ} 17^{\prime} 49,56^{\prime \prime} \mathrm{W}\right) .8\right)$ Morromico Norte $\left(5^{\circ} 48^{\prime} 47,16^{\prime \prime} \mathrm{N}\right.$, $77^{\circ} 17^{\prime} 49,56^{\prime \prime}$ W). 9) Punta Arusí (5 $36^{\prime} 39,996^{\prime \prime}$ N, $\left.77^{\circ} 29^{\prime} 6,36^{\prime \prime} \mathrm{W}\right)$. 10) Punta Orión ( $\left.5^{\circ} 37^{\prime} 50,664^{\prime \prime} \mathrm{N}, 77^{\circ} 23^{\prime} 18,59^{\prime \prime} \mathrm{W}\right)$. 11) Piedra de Jairo ( $\left.5^{\circ} 36^{\prime} 36,04^{\prime \prime} \mathrm{N}, 77^{\circ} 30^{\prime} 2,52^{\prime \prime} \mathrm{W}\right) .12$ ) Roñosa $5^{\circ} 35^{\prime} 6,22^{\prime \prime} \mathrm{N}$, $77^{\circ} 30^{\prime} 50,76^{\prime \prime}$ W). 13) Parguera-Corrientes ( $5^{\circ} 36^{\prime} 35,60^{\prime \prime} \mathrm{N}$, $\left.\left.77^{\circ} 30^{\prime} 14,76^{\prime \prime} \mathrm{W}\right) .14\right)$ Piedra de Colo ( $\left.5^{\circ} 30^{\prime} 43,87^{\prime \prime} \mathrm{N}, 77^{\circ} 32^{\prime} 1,32^{\prime \prime} \mathrm{W}\right)$.
Figure 1. Study area in the northern sector of the department of Chocó (Colombian Pacific). The sampling locations are numbered: 1) Piedra de Rodrigo (6 $6^{\circ} 47^{\prime} 2.07^{\prime \prime}$ N. $77^{\circ} 41^{\prime} 36.6^{\prime \prime}$ W). 2) Piedra de Eroito (6 $6^{\circ} 48^{\prime}$ $52.84^{\prime \prime}$ N. $77^{\circ} 41^{\prime} 32.99^{\prime \prime}$ W). 3) La Foca $\left(6^{\circ} 40^{\prime} 42.7044^{\prime \prime}\right.$ N. $77^{\circ} 41^{\prime}$ $42.71^{\prime \prime}$ W). 4) La Viuda (6 $37^{\prime} 58.872^{\prime \prime}$ N. $77^{\circ} 29^{\prime} 58.92^{\prime \prime}$ W). 5) La Mina ( $6^{\circ} 41^{\prime} 11.76^{\prime \prime}$ N. $77^{\circ} 32^{\prime} 57.48^{\prime \prime}$ W). 6) La Parguera-Piñas $\left(6^{\circ} 41^{\prime}\right.$ $30.084^{\prime \prime}$ N. $77^{\circ} 32^{\prime} 29.76^{\prime \prime}$ W). 7) Morros de Jurubidá (5 $48^{\prime} 47.16^{\prime \prime} \mathrm{N}$.

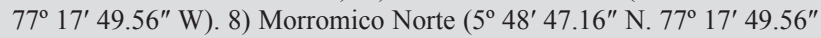
W). 9) Punta Arusí (5 $36^{\prime} 39.996^{\prime \prime}$ N. $77^{\circ} 29^{\prime} 6.36^{\prime \prime}$ W). 10) Punta Orión ( $5^{\circ} 37^{\prime} 50.664^{\prime \prime}$ N. $77^{\circ} 23^{\prime} 18.59^{\prime \prime}$ W). 11) Piedra de Jairo (5 $36^{\prime} 36.04^{\prime \prime}$ N. $77^{\circ} 30^{\prime} 2.52^{\prime \prime}$ W). 12) Roñosa $5^{\circ} 35^{\prime} 6.22^{\prime \prime}$ N. $77^{\circ} 30^{\prime} 50.76^{\prime \prime}$ W). 13) Parguera-Corrientes ( $5^{\circ} 36^{\prime} 35.60^{\prime \prime}$ N. $77^{\circ} 30^{\prime} 14.76^{\prime \prime}$ W). 14) Piedra de Colo ( $5^{\circ} 30^{\prime} 43.87^{\prime \prime}$ N. $\left.77^{\circ} 32^{\prime} 1.32^{\prime \prime} \mathrm{W}\right)$. y esqueletos, como se describe en Zea (1987), los cuales se observaron y fotografiaron empleando un microscopio óptico (Zeiss, Axio Lab. A1). Se midieron hasta 25 espículas de cada clase y las estructuras del esqueleto más relevantes. Las medidas de las espículas se presentan como mínimo-promedio-máximo de largo por ancho en $\mu \mathrm{m}$. Las
25 spicules of each class and the most relevant skeletal structures were measured. Spicule measurements are presented as minimum-average-maximum length $\mathrm{x}$ width in $\mu \mathrm{m}$. The identifications were carried out by consulting the keys of the Systema Porifera (Hooper et al., 2002) and the descriptions of materials from the Pacific, especially 
identificaciones se llevaron a cabo consultando las claves del Systema Porifera (Hooper et al., 2002) y las descripciones de material del Pacífico, especialmente del Pacífico Oriental, con la ayuda de las listas de especies del World Porifera Database (http://www.marinespecies.org/porifera; Van Soest et al., 2018). El material estudiado se depositó en la colección de esponjas (siglas INV POR) del Museo de Historia Natural Marina de Colombia (MHNMC) del Invemar mientras que la información se ingresó en las bases de datos del Sistema de Información en Biodiversidad Marina de Colombia (SIBM).

\section{RESULTADOS}

Se identificaron 24 especies de 13 órdenes, 17 familias y 21 géneros, de las cuales 21 son nuevos registros para el Pacífico colombiano (*). Doce (12) especies/ morfotipos quedaron identificados a nivel de género o con una asignación tentativa a nivel de especie. En la Tabla 1 se presenta la lista de especies y, a continuación, se adelanta la descripción de cada una.

Tabla 1. Lista de especies de esponjas en el Pacífico norte colombiano, agrupadas por orden. En negrita se indican las especies que tienen una contraparte morfológicamente similar en el Caribe. the Eastern Pacific, with the help of the species lists from the World Porifera Database (http://www.marinespecies. org/porifera; Van Soest et al., 2018). The material studied was deposited in the sponge collection (acronym INV POR) of the Museum of Marine Natural History of Colombia (MHNMC) of Invemar while the information was entered in the databases of the Information System on Marine Biodiversity of Colombia (SIBM).

\section{RESULTS}

24 species of 13 orders, 17 families, and 21 genera were identified, of which 21 are new records for the Colombian Pacific (*). Twelve (12) species/morphotypes were identified at the genus level or with a tentative assignment at the species level. In Table 1 the list of species is presented and the description of each one is given below.

\begin{tabular}{|c|c|}
\hline Orden/Order & Especie/Species \\
\hline AGELASIDA & 1. Prosuberites aff. laughlini (Díaz, Álvarez y Van Soest, 1987) \\
\hline \multirow{2}{*}{ AXINELLIDA } & 2. Axinella nayaritensis* Carballo, Bautista-Guerrero, Cruz-Barraza, 2018 \\
\hline & 3. Dragmacidon sp." \\
\hline \multirow{2}{*}{ BUBARIDA } & 4. Desmanthus levii Van Soest y Hajdu, 2000 \\
\hline & 5. Acanthella sp." \\
\hline CLIONAIDA & 6. Placospongia aff. intermedia* Bowerbank, 1862 \\
\hline HAPLOSCLERIDA & 7. Chalinula nematifera* De Laubenfels, 1954 \\
\hline \multirow{3}{*}{ POECILOSCLERIDA } & 8. Crambe panamensis* Maldonado, Carmona, Van Soest y Pomponi, 2001 \\
\hline & 9. Discorhabdella littoralis" Maldonado, Carmona, Van Soest y Pomponi, 2001 \\
\hline & 10. Clathria (Microciona) sp." \\
\hline \multirow{2}{*}{ SCOPALINIDA } & 11. Scopalina aff. ruetzleri" (Wiedenmayer, 1977) \\
\hline & 12. Scopalina sp. $2^{*}$ \\
\hline \multirow{3}{*}{ SUBERITIDA } & 13. Axinyssa isabela Carballo y Cruz-Barraza, 2008 \\
\hline & 14. Epipolasis sp." \\
\hline & 15. Terpios sp." \\
\hline TETRACTINELLIDA & 16. Geodia media* Bowerbank, 1873 \\
\hline \multirow{2}{*}{ DENDROCERATIDA } & 17. Aplysilla sp." \\
\hline & 18. Chelonaplysilla violacea* (Lendenfeld, 1883) sensu Gómez, Carballo, Vázquez y Cruz, 2002 \\
\hline DICTYOCERATIDA & 19. Scalarispongia similis (Thiele, 1905) \\
\hline CHONDROSIIDA & 20. Chondrosia tenochca* Carballo, Gómez, Cruz-Barraza y Flores-Sánchez, 2003 \\
\hline \multirow{4}{*}{ VERONGIIDA } & 21. Aplysina chiriquiensis* Díaz, Van Soest, Rützler y Guzmán, 2005 \\
\hline & 22. Aplysina gerardogreeni Gómez y Bakus, 1992 \\
\hline & 23. Aplysina cf. revillagigedi" Cruz-Barraza, Carballo, Rocha-Olivares, Ehrlich y Hog, 2012 \\
\hline & 24. Vansoestia sp." \\
\hline
\end{tabular}

Table 1. List of sponge species in the Colombian North Pacific, grouped by order. Species that have a morphologically similar counterpart in the Caribbean are indicated in bold. 
Clase Demospongiae Sollas, 1885

Subclase Heteroscleromorpha Cárdenas, Pérez y

Boury-Esnault, 2012

Orden Agelasida Hartman, 1980

Familia Hymerhabdiidae Morrow, Picton, Erpenbeck, Boury-Esnault, Maggs y Allcock, 2012

\section{Especie 1. Prosuberites aff. laughlini} (Díaz, Álvarez y Van Soest, 1987)

Figura 2; Lám. 1, figs. 1, 4.

?Eurypon laughlini Díaz, Álvarez y Van Soest, 1987: 33, Lám. I-A, fig. 2.

Eurypon aff. laughlini; Escobar, 2000: 62, figs. 22-23.

Prosuberites laughlini; Nichols, 2005: 91, Apéndice A

(Especímenes: UCMPWC952 y UCMPWC875).

Prosuberites aff. laughlini; García-Suárez et al. 2012: 21.

Material estudiado: INV POR1361, Punta Cruces, La Parguera-Piñas, 13 m, col. L. Chasqui, 16-08-2015. INV POR1375, golfo de Tribugá, Morromico, 11,9 m, col. L. Chasqui, 20-08-2015. INV POR1389, Punta Cruces, La Viuda, 13-15 m, col. L. Chasqui, 14-08-2015. INV POR1395, Punta Cruces, La Mina, 15 m, col. L. Chasqui, 16-08-2015. INV POR1407, cabo Marzo, Piedra de Rodrigo, 18,5 m, col. L. Chasqui, 15-08-2015.

Forma: revestimiento grueso, ampliamente extendido sobre el sustrato rocoso; superficie corrugada, con espículas erectas que atraviesan la piel; ósculos dispersos con un collar elevado transparente. Color: naranja brillante; marrón claro, a veces amarillo pálido en alcohol. Consistencia: suave, frágil; híspida al tacto. Ectosoma: pinacodermo delgado, atravesado por espículas grandes. Coanosoma: tractos erectos o espículas individuales saliendo de la base de fijación, 38$115 \mu \mathrm{m}$ de grosor, separados 46-230 $\mu \mathrm{m}$ (Figura 2C, 2D). Espículas: tiloestilos, $184-568-1221 \mu \mathrm{m}$ por $5,0-11,0-18,3$ $\mu \mathrm{m}$ (Figura 2A, 2B).

Hábitat: sustratos rocosos verticales expuestos y en paredes de cuevas.

Distribución en el Pacífico: Pacífico de Panamá (Nichols, 2005); isla de Malpelo (García-Suárez et al., 2012); isla Palma en bahía Málaga (Escobar, 2000); Pacífico norte colombiano.

Comentarios: al revisar las descripciones de Díaz et al. (1987) para especímenes del Caribe y de Escobar (2000) para especímenes del Pacífico, se puede hipotetizar que se trata
Class Demospongiae Sollas, 1885

Subclass Heteroscleromorpha Cárdenas, Pérez and Boury-Esnault, 2012

Order Agelasida Hartman, 1980

Family Hymerhabdiidae Morrow, Picton,

Erpenbeck, Boury-Esnault, Maggs and Allcock, 2012

\section{Species 1. Prosuberites aff. laughlini}

(Díaz, Álvarez and Van Soest, 1987)

Figure 2; Plate 1, figs. 1, 4.

?Eurypon laughlini Díaz, Álvarez and Van Soest, 1987: 33, Plate I-A, fig. 2.

Eurypon aff. laughlini; Escobar, 2000: 62, figs. 22-23.

Prosuberites laughlini; Nichols, 2005: 91, Appendix A

(Specimens: UCMPWC952 and UCMPWC875).

Prosuberites aff. laughlini; García-Suárez et al. 2012: 21.

Studied material: INV POR1361, Punta Cruces, La Parguera-Piñas, 13 m, col. L. Chasqui, 08-16-2015. INV POR1375, Gulf of Tribugá, Morromico, $11.9 \mathrm{~m}$, col. L. Chasqui, 08-20-2015. INV POR1389, Punta Cruces, La Viuda, 13-15 m, col. L. Chasqui, 08-14-2015. INV POR1395, Punta Cruces, La Mina, 15 m, col. L. Chasqui, 08-16-2015. INV POR1407, Cape Marzo, Piedra de Rodrigo, 18.5 m, col. L. Chasqui, 08-15-2015.

Shape: thick encrustation, widely spread on the rocky substratum; corrugated surface, with erect spicules traversing the skin; scattered oscules with a transparent raised collar. Color: bright orange; light brown, sometimes pale yellow in alcohol. Consistency: soft, fragile; hispid to the touch. Ectosome: thin pinacoderm, pierced by large spicules. Choanosome: erect tracts or individual spicules emerging from the base of fixation, 38-115 $\mu \mathrm{m}$ thick, separated 46-230 $\mu \mathrm{m}$ (Figure 2C, 2D). Spicules: tylostyles, 184-568-1221 $\mu \mathrm{m}$ by $5.0-11.0-18.3 \mu \mathrm{m}$ (Figure 2A, 2B).

Habitat: exposed vertical rock substrata and in cave walls.

Distribution in the Pacific: Pacific of Panama (Nichols, 2005); Malpelo Island (García-Suárez et al., 2012); Palma Island in Malaga Bay (Escobar, 2000); Colombian North Pacific.

Comments: when reviewing the descriptions by Díaz et al. (1987) for specimens from the Caribbean and Escobar (2000) for specimens from the Pacific, it can be hypothesized that these are populations of the same species 
de poblaciones de la misma especie que fueron separadas por el levantamiento del istmo de Panamá hace unos tres millones de años. $\mathrm{O}$, alternativamente, podría tratarse de una dispersión reciente a través del canal de Panamá. Aunque existen trabajos moleculares con la especie (Nichols, 2005), estos no permiten hacer comparaciones de las poblaciones a ambos lados del istmo (ver también Holmes y Blanch, 2007; Schuster et al., 2015). Deben realizarse estudios más detallados para determinar si hay diferencias morfológicas entre las poblaciones del Pacífico y el Caribe, y de ser diferentes, darle un nombre a la especie del Pacífico. GarcíaSuárez et al. (2012) registran Prosuberites aff. laughlini para la isla Malpelo con base en una fotografía submarina, por lo cual se incluye esa localidad aquí en la distribución de la especie; sin embargo, ese registro debe confirmarse con material biológico. that were separated by the uplift of the Isthmus of Panama about three million years ago. Alternatively, it could be a recent dispersal through the Panama Canal. Although there are molecular studies with the species (Nichols, 2005), these do not allow comparisons of the populations on both sides of the isthmus (see also Holmes and Blanch, 2007; Schuster et al., 2015). More detailed studies should be carried out to determine if there are morphological differences between the Pacific and Caribbean populations and if different, give the Pacific species a new name. García-Suárez et al. (2012) record Prosuberites aff. laughlini for Malpelo Island based on an underwater photograph, for which that locality is included here in the distribution of the species; however, that record must be confirmed with biological material.

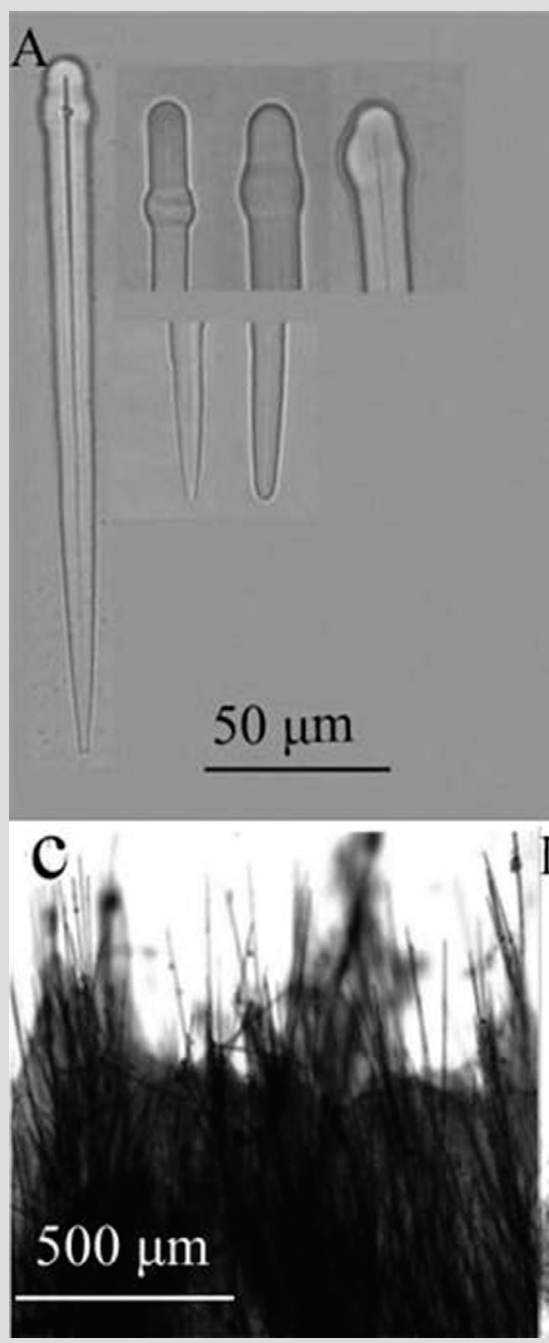

Figura 2. Prosuberites aff. laughlini. A) Tiloestilos con diferentes terminaciones en cabeza y punta. B) Tiloestilos. C) y D) Corte perpendicular mostrando ectosoma y coanosoma.

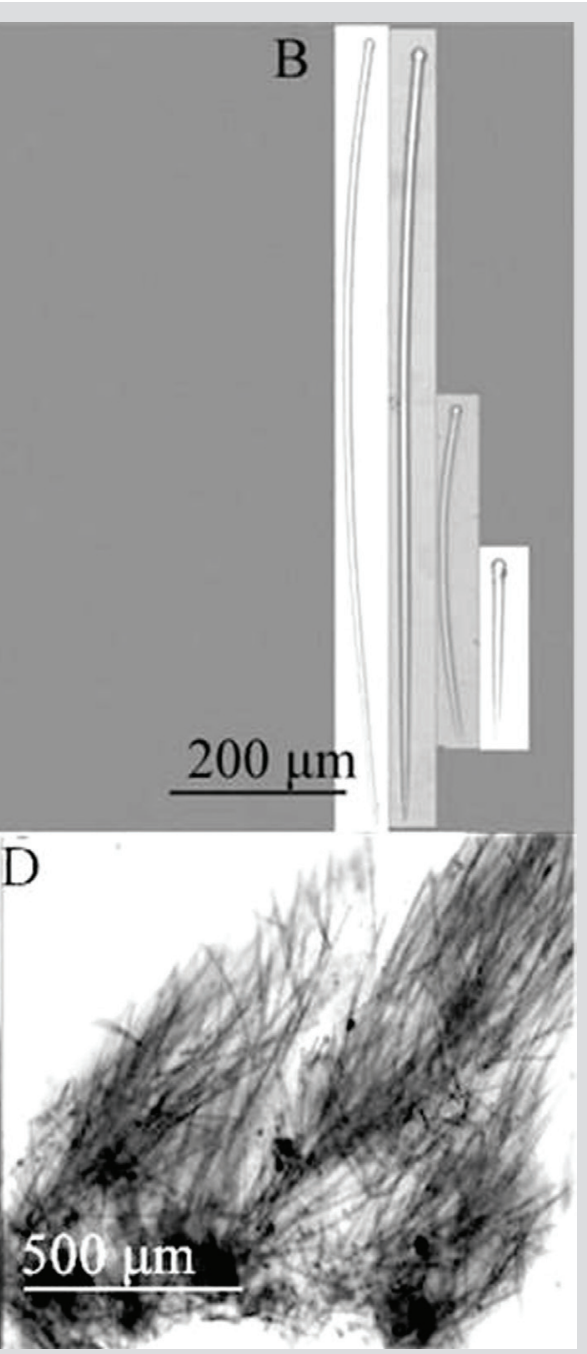

Figure 2. Prosuberites aff. laughlini. A) Tylostyles with different endings in head and tip. B) Tylostyles. C) and D) Perpendicular section showing ectosome and choanosome. 
Orden Axinellida Lévi, 1953

Familia Axinellidae Carter, 1875

Especie 2. Axinella nayaritensis Carballo, Bautista-Guerrero y Cruz-Barraza, 2018

Figura 3; Lám. 1, fig. 2.

Axinella nayaritensis Carballo, Bautista-Guerrero y CruzBarraza, 2018: 114, figs. 2-3.

Material estudiado: INV POR1372, golfo de Tribugá, Punta Orión, 7,8 m, col. L. Chasqui, 20-08-2015. INV POR1404, cabo Corrientes, La Roñosa, 14,4 m, col. L. Chasqui, 21-08-2015.

Forma: erecta, arborescente, $15-30 \mathrm{~cm}$ de altura; ramas como dedos, se van estrechando apicalmente; superficie muy irregular y corrugada. Color: naranja brillante; amarillo pálido en alcohol. Consistencia: firme, poco compresible. Ectosoma: pinacodermo sostenido por mechones de espículas del final de los tractos ascendentes. Coanosoma: tractos difusos de espículas, 32-139 $\mu \mathrm{m}$ de grosor, separados 46-223 $\mu \mathrm{m}$, interconectados irregularmente por espículas de forma dispersa. Los tractos ascienden desde la parte central de la rama y divergen hacia la superficie, llegando perpendicularmente a ella (Figura 3D, 3E). Espículas: oxeas, 241-362-632 $\mu \mathrm{m}$ por 6,9-11,5-17,5 $\mu \mathrm{m}$ (Figura 3A); estilos I: delgados y rectos, la punta se agudiza gradualmente, 223-307-388 $\mu \mathrm{m}$ por 7,8-12,4-17,8 $\mu \mathrm{m}$ (Figura 3B); estilos II: gruesos y ligeramente doblados a manera de rabdoestilo, 407-518-642 $\mu \mathrm{m}$ por 4,6-9,0-12,3 $\mu \mathrm{m}$ (Figura 3C).

Hábitat: en zonas poco expuestas del arrecife rocoso.

Distribución: Pacífico tropical mexicano (Carballo et al., 2018), Pacífico norte colombiano.

Comentarios: las espículas y la estructura del esqueleto son característicos de las especies arbustivas o ramosas del género Axinella Schmidt, 1862. Carballo et al. (2018) la describieron como nueva especie para el Pacífico tropical mexicano. Se enviaron fotografías in vivo y de material procesado en el laboratorio de los especímenes recolectados en este estudio al profesor J. L. Carballo, quien confirmó la similitud con A. nayaritensis.
Order Axinellida Lévi, 1953

Family Axinellidae Carter, 1875

Species 2. Axinella nayaritensis Carballo, Bautista-Guerrero and Cruz-Barraza, 2018

Figure 3; Plate 1, fig. 2.

Axinella nayaritensis Carballo, Bautista-Guerrero and Cruz-Barraza, 2018: 114, figs. 2-3.

Material studied: INV POR1372, Gulf of Tribugá, Punta Orión, 7.8 m, col. L. Chasqui, 08-20-2015. INV POR1404, Cape Corrientes, La Roñosa, 14.4 m, col. L. Chasqui, 0821-2015.

Shape: erect, arborescent, $15-30 \mathrm{~cm}$ tall; branches like fingers, tapering apically; very uneven and corrugated surface. Color: bright orange; pale yellow in alcohol. Consistency: firm, not very compressible. Ectosome: pinacoderm supported by tufts of spicules from the end of the ascending tracts. Choanosome: diffuse tracts of spicules, 32-139 $\mu \mathrm{m}$ thick, separated 46-223 $\mu \mathrm{m}$, irregularly interconnected by interspersed spicules. The tracts rise from the central part of the branch and diverge towards the surface, arriving perpendicular to it (Figure 3D, 3E). Spicules: oxeas, 241-362-632 $\mu \mathrm{m}$ by 6.9-11.5-17.5 $\mu \mathrm{m}$ (Figure 3A); styles I: thin and straight, the tip gradually tapers, 223-307-388 $\mu \mathrm{m}$ by 7.8-12.4-17.8 $\mu \mathrm{m}$ (Figure 3B); styles II: thick and slightly bent like a rhabdostyle, 407-518$642 \mu \mathrm{m}$ by 4.6-9.0-12.3 $\mu \mathrm{m}$ (Figure 3C).

Habitat: in non-exposed areas of the rocky reef.

Distribution: Mexican tropical Pacific (Carballo et al., 2018), Colombian North Pacific.

Comments: the spicules and the structure of the skeleton are characteristic of bushy or branchy species of the genus Axinella Schmidt, 1862. Carballo et al. (2018) described it as a new species for the Mexican tropical Pacific. In vivo photographs and laboratory-processed material of the specimens collected in this study were sent to Professor J. L. Carballo, who confirmed the similarity to $A$. nayaritensis. 


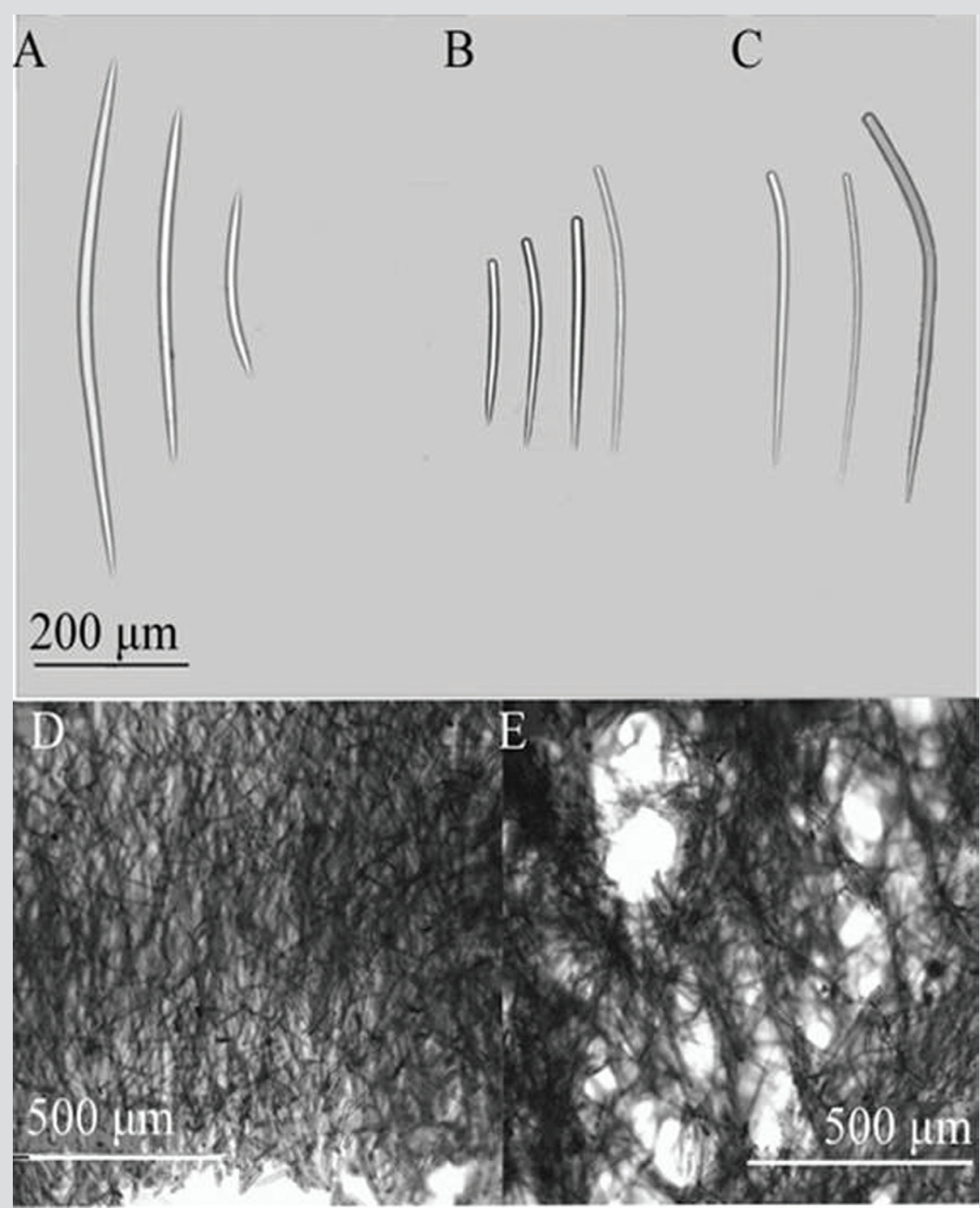

Figura 3. Axinella nayaritensis. A) Oxeas. B) Estilos I. C) Estilos II. D) y E) Tractos del coanosoma.

Figure 3. Axinella nayaritensis. A) Oxeas. B) Styles I. C) Styles II. D) and E) Choanosome tracts.

\section{Especie 3. Dragmacidon sp.}

Figura 4; Lám. 1, fig. 3.

Material estudiado: INV POR1362, Punta Cruces, La Parguera-Piñas, 6 m, col. L. Chasqui, 16-08-2015.

Forma: revestimiento grueso (varios $\mathrm{mm}$ ), $10 \times 5 \mathrm{~cm}$; superficie lisa, ósculos dispersos. Color: rojo escarlata; amarillo pálido en alcohol. Consistencia: firme, poco compresible. Ectosoma: pinacodermo orgánico sostenido por mechones de espículas de los tractos ascendentes. Coanosoma: red prismática de tractos espiculares plumosos ascendentes y divergentes, 181-549 $\mu \mathrm{m}$ de grosor, separados 74-155 $\mu \mathrm{m}$, interconectados por tractos transversales, 79-133 $\mu \mathrm{m}$ de grosor (Figura 4C, 4D); ojo de malla 119-255 $\mu \mathrm{m}$ (Figura 4B). Espículas: estilos curvos y

\section{Species 3. Dragmacidon sp.}

Figure 4; Plate 1, fig. 3.

Material studied: INV POR1362, Punta Cruces, La Parguera-Piñas, 6 m, col. L. Chasqui, 08-16-2015.

Shape: thick encrustation (several $\mathrm{mm}$ ), $10 \times 5 \mathrm{~cm}$; smooth surface, scattered oscules. Color: scarlet red; pale yellow in alcohol. Consistency: firm, not very compressible. Ectosome: organic pinacoderm supported by tufts of spicules from the ascending tracts. Choanosome: a prismatic network of ascending and divergent plumose spicular tracts, 181-549 $\mu \mathrm{m}$ thick, separated 74-155 $\mu \mathrm{m}$, interconnected by transverse tracts, 79-133 $\mu \mathrm{m}$ thick (Figure 4C, 4D); mesh size 119-255 $\mu \mathrm{m}$ (Figure 4B). Spicules: curved and thick styles, folded in a rhabdostyle fashion, with the head 


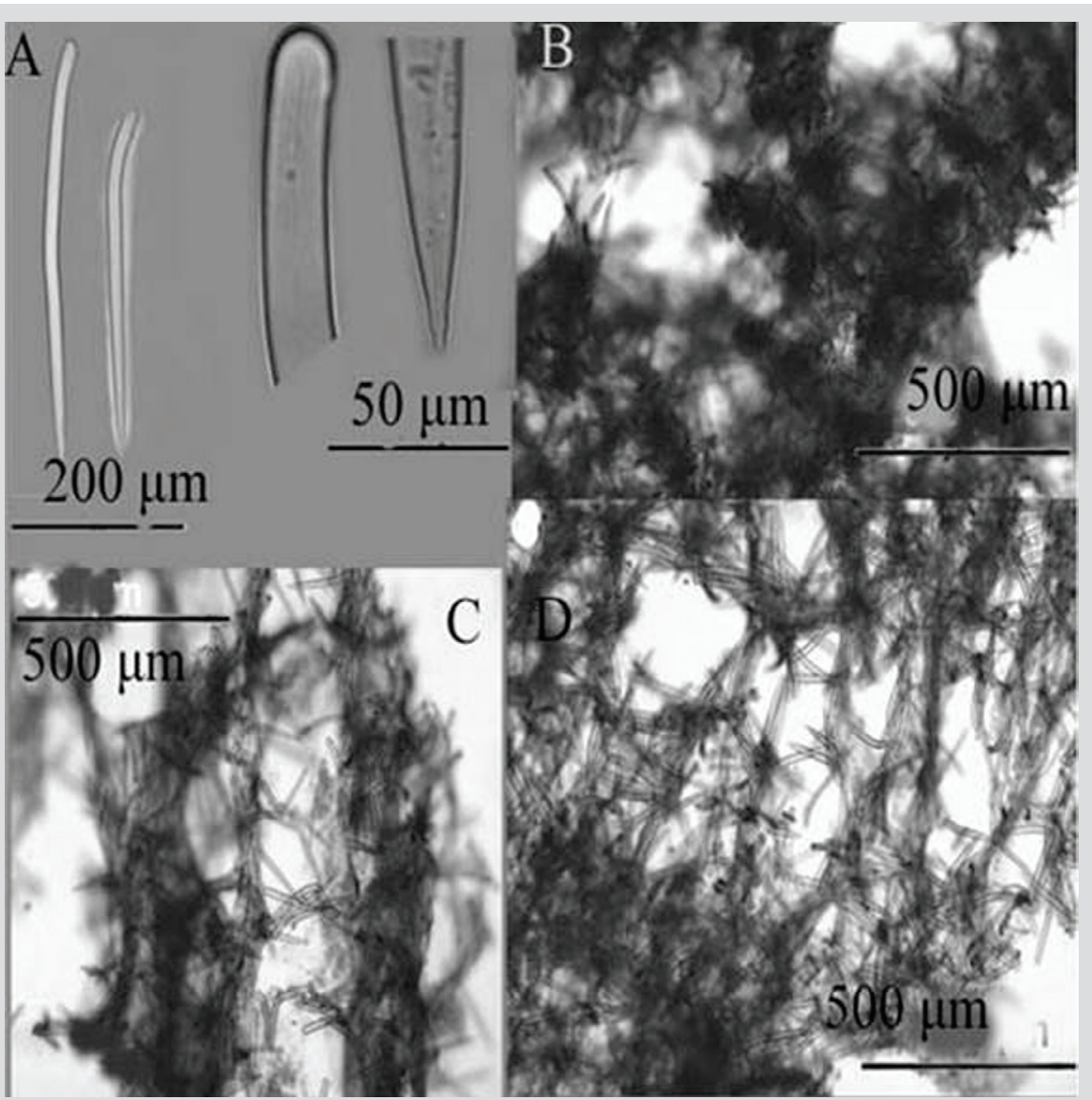

Figura 4. Dragmacidon sp. A) Estilos. B) Esqueleto, vista tangencial. C) y D) Tractos del coanosoma.
Figure 4. Dragmacidon sp. A) Styles. B) Skeleton, tangential view. C) and D) Choanosome tracts. gruesos, doblados a manera de rabdoestilo, con la cabeza ligeramente pronunciada, $152-302-374 \mu \mathrm{m}$ por $8,7-18,8$ 26,1 $\mu \mathrm{m}$ (Figura 4A).

Hábitat: arrecife rocoso somero.

Distribución: Pacífico norte colombiano.

Comentarios: el espécimen se ubicó en este género por la forma de crecimiento, el color y la organización del coanosoma (Álvarez y Hooper, 2002), a pesar de no presentar oxeas. Austin et al. (2013) describieron una nueva especie de Columbia Británica y aguas adyacentes en Canadá y la llamaron Dragmacidon kishinensis, pero sus características no concuerdan con el material de Colombia. Por esta razón, se debe hacer un estudio más detallado (incluyendo marcadores moleculares) para confirmarla como nueva especie. slightly pronounced, $152-302-374 \mu \mathrm{m}$ by $8.7-18.8-26.1 \mu \mathrm{m}$ (Figure 4A).

Habitat: shallow rocky reef.

Distribution: Colombian North Pacific.

Comments: the specimen was placed in this genus due to its growth form, the color, and the organization of the choanosome (Álvarez and Hooper, 2002), despite not presenting oxeas. Austin et al. (2013) described a new species from British Columbia and adjacent waters in Canada and named it Dragmacidon kishinensis, but its characteristics do not match material from Colombia. For this reason, a more detailed study (including molecular markers) must be done to confirm it as a new species. 
Orden Bubarida Morrow y Cárdenas, 2015

Familia Desmanthidae Topsent, 1893

\section{Especie 4. Desmanthus levii Van Soest y Hajdu, 2000}

Figura 5; Lám. 1, fig. 4.

Desmanthus levii Van Soest y Hajdu, 2000: 22, figs. 1F-I.

Material estudiado: INV POR1376, golfo de Tribugá, Morromico, 12 m, col. L. Chasqui, 20-08-2015.

Forma: revestimiento delgado recubriendo ampliamente el sustrato; superficie lisa, finamente granulada.

Color: naranja rojizo, púrpura opaco en alcohol. Consistencia: indefinida por lo delgada. Ectosoma: pinacodermo perforado por estilos largos, erectos, dispuestos entre desmas II. Coanosoma: capa basal de desmas I, seguida por una o varias capas de desmas II, con el cladoma predominantemente orientado hacia arriba y el rabdoma hacia abajo; estilos erectos dispersos (Figura 5E). Espículas: estilos lisos, 177-434-846 $\mu \mathrm{m}$ por 4,5-8,5-16,5 $\mu \mathrm{m}$, rectos o curvados a manera de rabdoestilos (Figura 5A, 5B); desmas I: múltiples ramificaciones delgadas, 56$100-141 \mu \mathrm{m}$ por $5,2-10,8-18,8 \mu \mathrm{m}$ (Figura $5 \mathrm{D}$ ); desmas II (tetracrépidas): rabdomas cónicos, robustos, en forma de zanahoria, 43-72-112 $\mu \mathrm{m}$ por 10,5-20,6-36,7 $\mu \mathrm{m}$, cladoma 60-93-166 $\mu \mathrm{m}$ por 21-15-10 $\mu \mathrm{m}$ (Figura $5 \mathrm{C}$ ).

Hábitat: paredes de arrecife rocoso en aguas poco profundas.

Distribución: costa Pacífica de Panamá (Van Soest y Hajdu, 2000); Pacífico norte colombiano.

Comentarios: la identificación se realizó con base en el trabajo previo de Van Soest y Hajdu (2000) de la costa Pacífica de Panamá. Es posible que Desmanthus incrustans (Topsent, 1889) del Caribe sea una especie hermana de $D$. levii por su similitud en la morfología y estructura espicular; sin embargo, se requieren trabajos comparativos con marcadores moleculares para establecer esa afinidad.
Order Bubarida Morrow and Cárdenas, 2015

Family Desmanthidae Topsent, 1893

\section{Species 4. Desmanthus levii Van Soest and Hajdu, 2000}

Figure 5; Plate 1, fig. 4.

Desmanthus levii Van Soest and Hajdu, 2000: 22, figs.

1F-I.

Studied material: INV POR1376, Gulf of Tribugá, Morromico, 12 m, col. L. Chasqui, 08-20-2015.

Shape: thin encrustation broadly covering the substratum; smooth, finely grained surface.

Color: reddish-orange, dull purple in alcohol. Consistency: undefined from being thin. Ectosome: pinacoderm pierced by long, erect styles, arranged between desmas II. Choanosome: a basal layer of desmas I, followed by one or more layers of desmas II, with the cladome predominantly facing upward and the rhabdom downward; scattered erect styles (Figure 5E). Spicules: smooth styles, 177-434-846 $\mu \mathrm{m}$ by $4.5-8.5-16.5 \mu \mathrm{m}$, straight or curved in a rhabdostyle fashion (Figure 5A, 5B); Desmas I: multiple thin branches, 56-100-141 $\mu \mathrm{m}$ by 5.2-10.8-18.8 $\mu \mathrm{m}$ (Figure 5D); Desmas II (tetracrepids): conical rhabdomes, robust, carrot-shaped, 43-72-112 $\mu \mathrm{m}$ by 10.5-20.6-36.7 $\mu \mathrm{m}$, cladome 60-93-166 $\mu \mathrm{m}$ by $21-15-10 \mu \mathrm{m}$ (Figure $5 \mathrm{C}$ ).

Habitat: rocky reef walls in shallow water.

Distribution: Pacific coast of Panama (Van Soest and Hajdu, 2000); Colombian North Pacific

Comments: the identification was made based on the previous work of Van Soest and Hajdu (2000) of the Pacific coast of Panama. It is possible that Desmanthus incrustans (Topsent, 1889) from the Caribbean is a sister species to D. levii due to its similarity in morphology and spicular structure; however, comparative work with molecular markers is required to establish this affinity. 


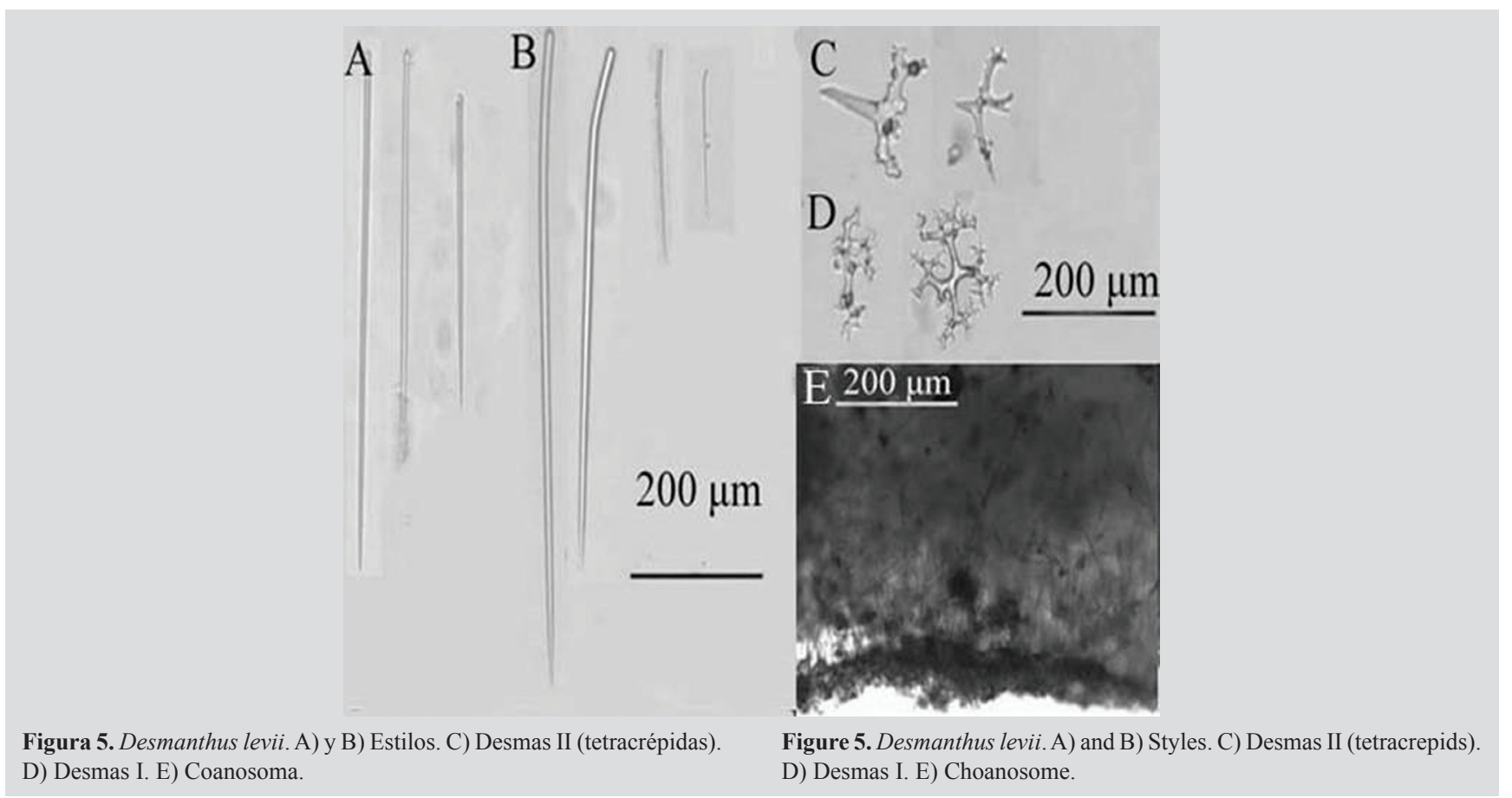

Familia Dictyonellidae Van Soest, Díaz y

Pomponi, 1990

\section{Especie 5. Acanthella sp.}

Figura 6; Lám. 1, fig. 5.

Material estudiado: INV POR1381, golfo de Tribugá, Morros de Jurubidá, 12 m, col. L. Chasqui, 20-08-2015. INV POR1383, Morros de Jurubidá, 13,3 m, col. L. Chasqui, 2008-2015. INV POR1386, Morros de Jurubidá, 10 m, col. L. Chasqui, 20-08-2015. INV POR1373, golfo de Tribugá, Punta Orión, 7,1 m, col. L. Chasqui, 20-08-2015.

Forma: masa incrustante con lóbulos a arbustos bajos de hasta unos $5 \mathrm{~cm}$ de altura; ramitas de $1-5 \mathrm{~mm}$ de diámetro, poco divididas, saliendo casi desde la base; superficie aterciopelada o corrugada, microhíspida, frecuentemente con sedimento atrapado, especialmente hacia las bases. Color: naranja brillante; amarillo pálido en alcohol. Consistencia: suave, compresible. Ectosoma: pinacodermo sostenido por tractos erectos y espículas tangenciales. Coanosoma: tractos plumosos de espículas, 42-138 $\mu \mathrm{m}$ de grueso (Figura 6E), dendríticos, que divergen desde el centro de las ramitas hacia la superficie, llegando a esta diagonalmente, interconectados muy irregularmente por espículas sencillas en todas las direcciones. Espículas: estilos lisos y rectos, los más pequeños ligeramente curvados, 348-590-813 $\mu \mathrm{m}$ por 4,4-9,0-15,1 $\mu \mathrm{m}$ (Figura 6B, 6C, 6D); estrongilos sinuosos, $36-651-910 \mu \mathrm{m}$ por 3,6-6,0-9,6 $\mu \mathrm{m}$ (Figura $6 \mathrm{~A}$ ).
Family Dictyonellidae Van Soest, Díaz and Pomponi, 1990

\section{Species 5. Acanthella sp.}

Figure 6; Plate 1, fig. 5.

Material studied: INV POR1381, Gulf of Tribugá, Morros de Jurubidá, $12 \mathrm{~m}$, col. L. Chasqui, 08-20-2015. INV POR1383, Morros de Jurubidá, $13.3 \mathrm{~m}$, col. L. Chasqui, 0820-2015. INV POR1386, Morros de Jurubidá, $10 \mathrm{~m}$, col. L. Chasqui, 08-20-2015. INV POR1373, Gulf of Tribugá, Punta Orión, 7.1 m, col. L. Chasqui, 08-20-2015.

Shape: encrusting mass with lobes to low bushes up to about $5 \mathrm{~cm}$ in height; branches $1-5 \mathrm{~mm}$ in diameter, little divided, coming out almost from the base; velvety or corrugated surface, microhispid, often with trapped sediment, especially towards the base. Color: bright orange; pale yellow in alcohol. Consistency: soft, compressible. Ectosome: pinacoderm supported by erect tracts and tangential spicules. Choanosome: plumose spicule tracts, 42-138 $\mu \mathrm{m}$ thick (Figure 6E), dendritic, diverging from the center of the branches towards the surface, reaching it diagonally, interconnected very irregularly by simple spicules in all directions. Spicules: smooth and straight styles, the smallest slightly curved, $348-590-813 \mu \mathrm{m}$ by 4.49.0-15.1 $\mu \mathrm{m}$ (Figure 6B, 6C, 6D); sinuous strongyles, 36651-910 $\mu \mathrm{m}$ by 3.6-6.0-9.6 $\mu \mathrm{m}$ (Figure $6 \mathrm{~A}$ ). 


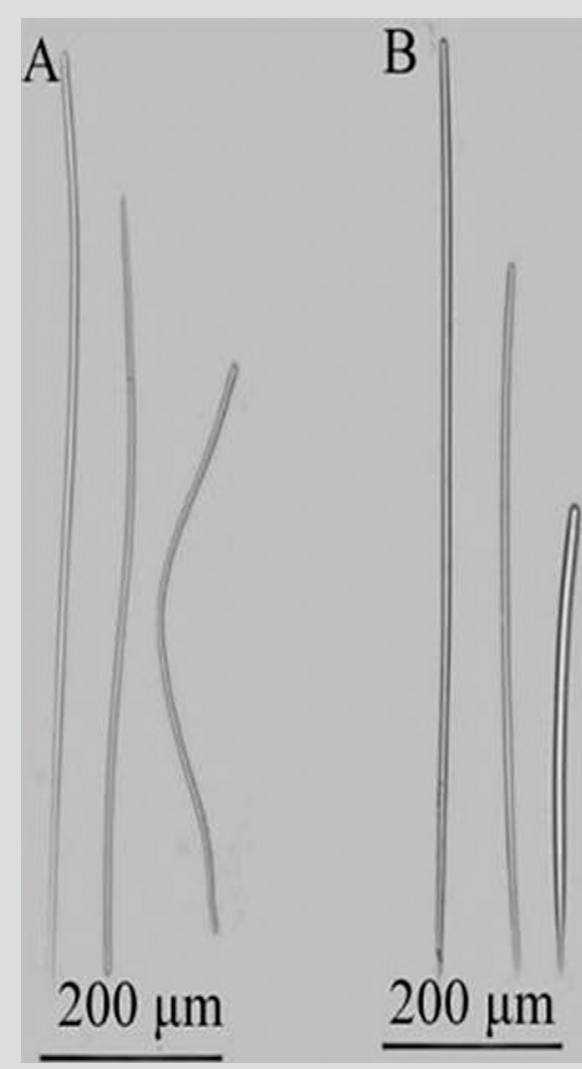

Figura 6. Acanthella sp. A) Estrongilos sinuosos. B) Estilos. C) Extremos de estrongilos sinuosos. D) Extremos de estilos. E) Sección transversal del esqueleto.

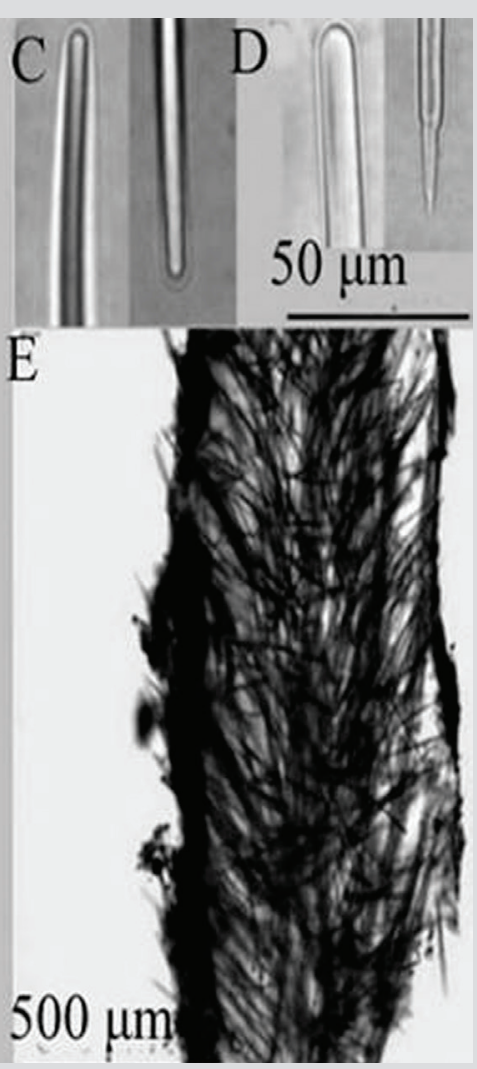

Figure 6. Acanthella sp. A) Sinuous strongyles. B) Styles. C) Winding strongyle ends. D) Extremes of styles. E) Cross-section of the skeleton.
Hábitat: arrecifes rocosos someros en aguas turbias.

Distribución: Pacífico norte colombiano.

Comentarios: la ubicación genérica de esta esponja es tentativa, con base en la presencia de tractos dendríticos y espículas de estilos curvos y estrongilos sinuosos (Van Soest et al., 2002), aunque hay otros géneros con ese mismo tipo de espiculación (v. g. Auletta Schmidt, 1870, Phakellia Bowerbank, 1862, Phycopsis Carter, 1883), pero del orden Axinellida (Van Soest et al., 2018). Acanthella danerii Costa, Barvastrello, Pansini y Bertolino, 2020 se describió de los fiordos chilenos en el Pacífico suroriental. Difiere de Acanthella sp. en tener oxeas sinuosas, curvas o dobladas, en adición a los estilos y los estrongilos sinuosos (Costa et al., 2020). La estructura del esqueleto en tractos plumosos y las espículas que presenta son características muy similares a Acanthella cubensis (Alcolado, 1984) del Caribe, pero difiere de ella en la forma de crecimiento. Debe ser sometida a una revisión más exhaustiva para considerarla como una nueva especie, ya que hay varias especies del género descritas para el Pacífico Occidental y el océano Índico (Van Soest et al., 2018; Costa et al., 2020).
Habitat: shallow rocky reefs in murky waters.

Distribution: Colombian North Pacific.

Comments: the generic location of this sponge is tentative, based on the presence of dendritic tracts and spicules with curved styles and sinuous strongyles (Van Soest et al., 2002), although there are other genera with the same type of spiculation (e.g. Auletta Schmidt, 1870, Phakellia Bowerbank, 1862, Phycopsis Carter, 1883), but of the order Axinellida (Van Soest et al., 2018). Acanthella danerii Costa, Barvastrello, Pansini, and Bertolino, 2020 was described from the Chilean fjords in the southeastern Pacific. It differs from Acanthella sp. in having sinuous, curved, or bent oxeas, in addition to sinuous styles and strongyles (Costa et al., 2020). The structure of the skeleton in plumose tracts and the spicules it presents are very similar characteristics to Acanthella cubensis (Alcolado, 1984) from the Caribbean, but it differs from it in the shape of growth. It should be subjected to a more exhaustive review to consider it as a new species since there are several species of the genus described for the Western Pacific and the Indian Ocean (Van Soest et al., 2018; Costa et al., 2020). 
Orden Clionaida Morrow y Cárdenas, 2015

Familia Placospongiidae Gray, 1867

Especie 6. Placospongia aff. intermedia Sollas, 1888

Figura 7.

?Placospongia intermedia Sollas, 1888: 273 (especie válida del Caribe).

Placospongia intermedia; De Laubenfels, 1936b: 454.

Material estudiado: INV POR1364, Punta Cruces, La Parguera-Piñas, 6 m, col. L. Chasqui, 16-08-2015.

Forma: incrustante, superficie dividida en placas poligonales por elevaciones irregulares o surcos. Color: rosa preservada en alcohol. Consistencia: dura, tenaz. Ectosoma: placas formadas por selenásteres densamente agrupados, de hasta 2 $\mathrm{mm}$ de grosor. Coanosoma: capa basal de selenásteres, 202$341 \mu \mathrm{m}$ de grosor, de la que se elevan tractos multiespiculados de tiloestilos, 137-290 $\mu \mathrm{m}$ de grosor, separados 163-711 $\mu \mathrm{m}$, a manera de columnas, que sostienen las placas ectosómicas. Numerosos selenásteres dispersos entre las columnas (Figura 7D, 7E). Espículas: megascleras: tiloestilos, 180-657$874 \mu \mathrm{m}$ por 5,2-10,6-17,6 $\mu \mathrm{m}$ (Figura 7B). Microscleras: esferásteres, 9,2-10,9-13,6 $\mu \mathrm{m}$ de diámetro (estadíos de desarrollo 16,1-18,3-19,5 $\mu \mathrm{m}$ ) (Figura 7A); selenásteres en varios estadíos de desarrollo, pequeños, 20,3-26,5-32,8 $\mu \mathrm{m}$ por 7,3-10-13,1 $\mu \mathrm{m}$ (Figura 7C-i); medianos, 43-36-55 $\mu \mathrm{m}$ por 16,9-25,0-33,4 $\mu \mathrm{m}$ (Figura 7C-ii); y desarrollados, 5566-76 $\mu \mathrm{m}$ por 42-52-62 $\mu \mathrm{m}$ (Figura $7 \mathrm{C}-$ iii).

Hábitat: sobre sustratos rocosos someros.

Distribución: Pacífico de Panamá (De Laubenfels, 1936b); Pacífico norte colombiano.

Comentarios: Sollas (1888) describió Placospongia intermedia de Punta Arenas, que luego fue confundida con una localidad con ese nombre en el Pacífico de Costa Rica. Sin embargo, en la sección "Species of the faunistic provinces", p. 383, Sollas (1888) localiza a $P$. intermedia en la provincia Caribe. En el World Porifera Database (http://www.marinespecies.org/porifera/porifera. $\mathrm{php}$ ? $\mathrm{p}=$ distribution\&id $=651010)$ se ubica la localidad tipo de P. intermedia en Punta Arenas, isla de Margarita. De Laubenfels (1936b) describió el material de $P$. intermedia tanto del lado Pacífico como del Caribe del istmo de Panamá, considerándolos la misma especie. Van Soest (2009) argumentó que el material de De Laubenfels del Caribe era diferente al del Pacífico, pero Rützler et al.
Order Clionaida Morrow and Cárdenas, 2015

Family Placospongiidae Gray, 1867

\section{Species 6. Placospongia aff. intermedia Sollas, 1888}

Figure 7.

?Placospongia intermedia Sollas, 1888: 273 (valid species from the Caribbean).

Placospongia intermedia; De Laubenfels, 1936b: 454.

Material studied: INV POR1364, Punta Cruces, La Parguera-Piñas, 6 m, col. L. Chasqui, 08-16-2015.

Shape: encrusting, surface divided into polygonal plates by irregular elevations or grooves. Color: rose preserved in alcohol. Consistency: tough, tenacious. Ectosome: plates formed by densely packed selenasters, up to $2 \mathrm{~mm}$ thick. Choanosome: basal layer of selenasters, 202-341 $\mu \mathrm{m}$ thick, from which multispicular tylostyle tracts arise, 137-290 $\mu \mathrm{m}$ thick, separated 163-711 $\mu \mathrm{m}$, like columns, supporting the ectosomal plates. Numerous selenasters are scattered among the columns (Figure 7D, 7E). Spicules: megascleres: tylostyles, $180-657-874 \mu \mathrm{m}$ by $5.2-10.6-17.6$ $\mu \mathrm{m}$ (Figure 7B). Microscleres: spherasters, 9.2-10.9-13.6 $\mu \mathrm{m}$ in diameter (developmental stages 16.1-18.3-19.5 $\mu \mathrm{m}$ ) (Figure 7A); selenasters in various stages of development, small, 20.3-26.5-32.8 $\mu \mathrm{m}$ by 7.3-10-13.1 $\mu \mathrm{m}$ (Figure 7C-i); medium, 43-36-55 $\mu \mathrm{m}$ by 16.9-25.0-33.4 $\mu \mathrm{m}$ (Figure 7Cii); and developed, 55-66-76 $\mu \mathrm{m}$ by $42-52-62 \mu \mathrm{m}$ (Figure 7C-iii).

Habitat: on shallow rocky substrata.

Distribution: Pacific of Panama (De Laubenfels, 1936b); Colombian North Pacific.

Comments: Sollas (1888) described Placospongia intermedia from Punta Arenas, which was later confused with a locality of that name in the Pacific of Costa Rica. However, in the section "Species of the faunistic provinces", p. 383, Sollas (1888) located P. intermedia in the Caribbean province. In the World Porifera Database (http://www.marinespecies.org/porifera/porifera.php?p= distribution\&id=651010) the type locality of $P$. intermedia is located in Punta Arenas, Margarita Island. De Laubenfels (1936b) described the material of $P$. intermedia from both the Pacific and Caribbean sides of the isthmus of Panama, considering them the same species. Van Soest (2009) argued that the De Laubenfels material from the Caribbean was different from that from the Pacific, but Rützler et al. 
(2014: 35) revisaron sus láminas originales sin encontrar diferencias. Es posible que el material del Pacífico sea una especie diferente que requiera de un nombre nuevo, aunque Nichols y Barnes (2005) no encontraron, a partir de análisis molecular de la región ITS que codifica en el ARN ribosomal, diferencias importantes entre varios especímenes del Caribe y del Pacífico de Panamá (Clado C8, sin identificar). Esto podría indicar que las dos poblaciones no han divergido lo suficiente desde que fueron separadas por el istmo, pero será necesario examinar morfológicamente ese material para llegar a una conclusión más precisa. En el Pacífico colombiano, Escobar (2000) describió P. carinata (Bowerbank, 1858), la cual es indudablemente otra especie, pues carece de esferásteres y su coloración es café oscuro. El registro de una fotografía submarina de Placospongia carinata de García-Suárez et al. (2012) de bahía Málaga no puede confirmarse por no haber material para examinar.
(2014: 35) reviewed the original slides without finding differences. The Pacific material may be a different species that requires a new name, although Nichols and Barnes (2005) did not find, from molecular analysis of the ITS region that encodes ribosomal RNA, important differences between various specimens of the Caribbean and Pacific of Panama (Clade C8, unidentified). This could indicate that the two populations have not diverged enough since they were separated by the isthmus, but it will be necessary to examine this material morphologically to reach a more precise conclusion. In the Colombian Pacific, Escobar (2000) described P. carinata (Bowerbank, 1858), which is undoubtedly another species, since it lacks spheresters and its color is dark brown. The record of an underwater photograph of Placospongia carinata from García-Suárez et al. (2012) from Bahía Málaga cannot be confirmed as there is no material to examine.

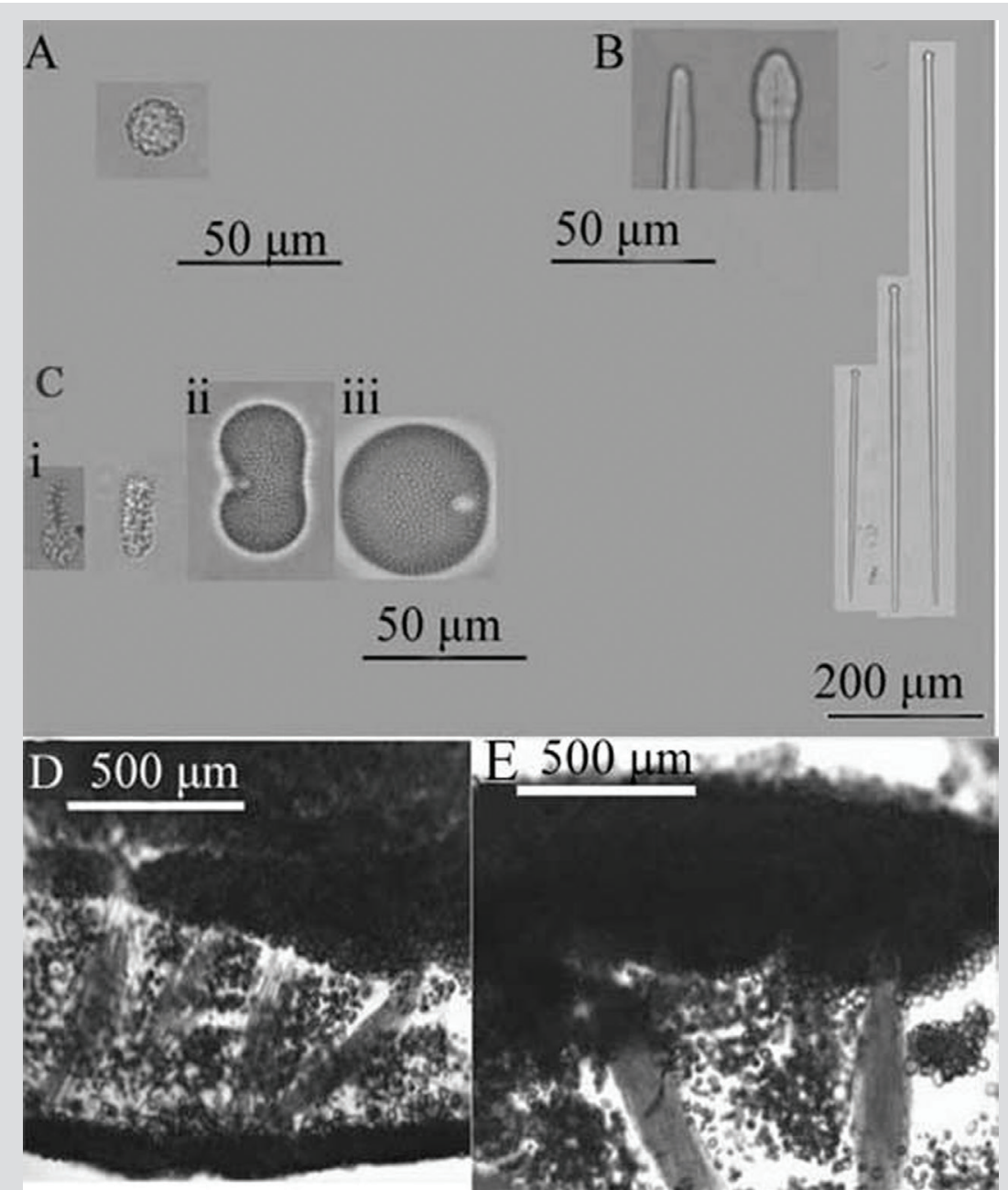

Figura 7. Placospongia aff. intermedia. A) Esferáster. B) Extremos de tiloestilos y tiloestilos. C) Selenásteres (i, ii, iii estadios). D) y E) Coanosoma.
Figure 7. Placospongia aff. intermediate. A) Spheraster. B) Ends of tylostyles and tylostyles. C) Selenasters (i, ii, iii stages). D) and E) Choanosome. 
Orden Haplosclerida Topsent, 1928

Familia Chalinidae Gray, 1867

\section{Especie 7. Chalinula nematifera}

(De Laubenfels, 1954)

Figura 8.

Nara nematifera De Laubenfels, 1954: 76, fig. 46.

Chalinula nematifera; De Weerdt, 2002: 853; Cruz-Barraza

y Carballo, 2008: 748, figs. 5, 7C; Vega, 2012: 105, figs.

7.2.57. A, B, C (transferencia de género).

Material estudiado: INV POR1380, golfo de Tribugá, Morromico, 12 m, col. L. Chasqui, 20-08-2015.

Forma: revestimiento a manera de cojín delgado, de aprox. $1 \mathrm{~cm}$ de diámetro. Superficie finamente híspida. Color: púrpura claro en alcohol. Consistencia: suave y compresible. Ectosoma: sin red ectosomal tangencial; formado por extremos de fibras ascendentes uniespiculares. Coanosoma: red cuadrada a poligonal de fibras de espongina rellenas por una o dos espículas, 6-22 $\mu \mathrm{m}$ de grosor, con mallas de 65-326 $\mu \mathrm{m}$ de diámetro (Figura 8B, 8C). Espículas: oxeas, $63-80-94 \mu \mathrm{m}$ por 2,6-3,7-5,1 $\mu \mathrm{m}$ (Figura 8A).

Hábitat: arrecifes rocosos someros.

Distribución: Pacífico central y occidental tropical (De Laubenfels, 1954); mar de Cortez (México) (Cruz-Barraza y Carballo, 2008); Pacífico norte colombiano.

Comentarios: fue descrita por primera vez por De Laubenfels (1954:76) como Nara nematifera con material del Pacífico centro-occidental. Luego, De Weerdt (2002) la transfirió al género Chalinula, que se define por tener un crecimiento finamente incrustante, en forma de almohadilla, y presentar oxeas cortas, que van desde vestigios a cigarros. La identificación de esta especie se realizó comparando las características que describen Cruz-Barraza y Carballo (2008) del Pacífico mexicano. Según Ávila y Carballo (2009), C. nematifera es una especie invasora nativa del Pacífico central y occidental que se registró desde 2003 en el Pacífico mexicano, donde se considera una amenaza potencial para los arrecifes coralinos por crecer sobre colonias vivas de Pocillopora spp.
Order Haplosclerida Topsent, 1928

Family Chalinidae Gray, 1867

\section{Species 7. Chalinula nematifera} (De Laubenfels, 1954)

Figure 8.

Nara nematifera De Laubenfels, 1954: 76, fig. 46.

Chalinula nematifera; De Weerdt, 2002: 853; Cruz-Barraza and Carballo, 2008: 748, figs. 5.7. C; Vega, 2012: 105, figs. 7.2.57. A, B, C (genus transfer).

Material studied: INV POR1380, Gulf of Tribugá, Morromico, 12 m, col. L. Chasqui, 08-20-2015.

Shape: encrusting thin cushion, approx. $1 \mathrm{~cm}$ in diameter. Finely hispid surface. Color: light purple in alcohol. Consistency: soft and compressible. Ectosome: lacking an ectosomal tangential network; formed by unispicular ascending fiber ends. Choanosome: square to a polygonal network of spongin fibers cored with one or two spicules, 6-22 $\mu \mathrm{m}$ thick, with meshes $65-326 \mu \mathrm{m}$ in diameter (Figure 8B, 8C). Spicules: oxeas, 63-80-94 $\mu \mathrm{m}$ by 2.6-3.7-5.1 $\mu \mathrm{m}$ (Figure 8A).

Habitat: shallow rocky reefs.

Distribution: Tropical central and western Pacific (De Laubenfels, 1954); Sea of Cortez (Mexico) (Cruz-Barraza and Carballo, 2008); Colombian North Pacific.

Comments: it was first described by De Laubenfels (1954: 76) as Nara nematifera with material from the centralwestern Pacific. Later, De Weerdt (2002) transferred it to the genus Chalinula, which is defined as having a finely encrusting growth form, in pillow-shape, and presenting short oxeas, ranging from vestiges to cigars. The identification of this species was carried out by comparing the characteristics described by Cruz-Barraza and Carballo (2008) of the Mexican Pacific. According to Ávila and Carballo (2009), C. nematifera is an invasive species native to the central and western Pacific that has been recorded since 2003 in the Mexican Pacific, where it is considered a potential threat to coral reefs because it grows on live Pocillopora spp. colonies. 


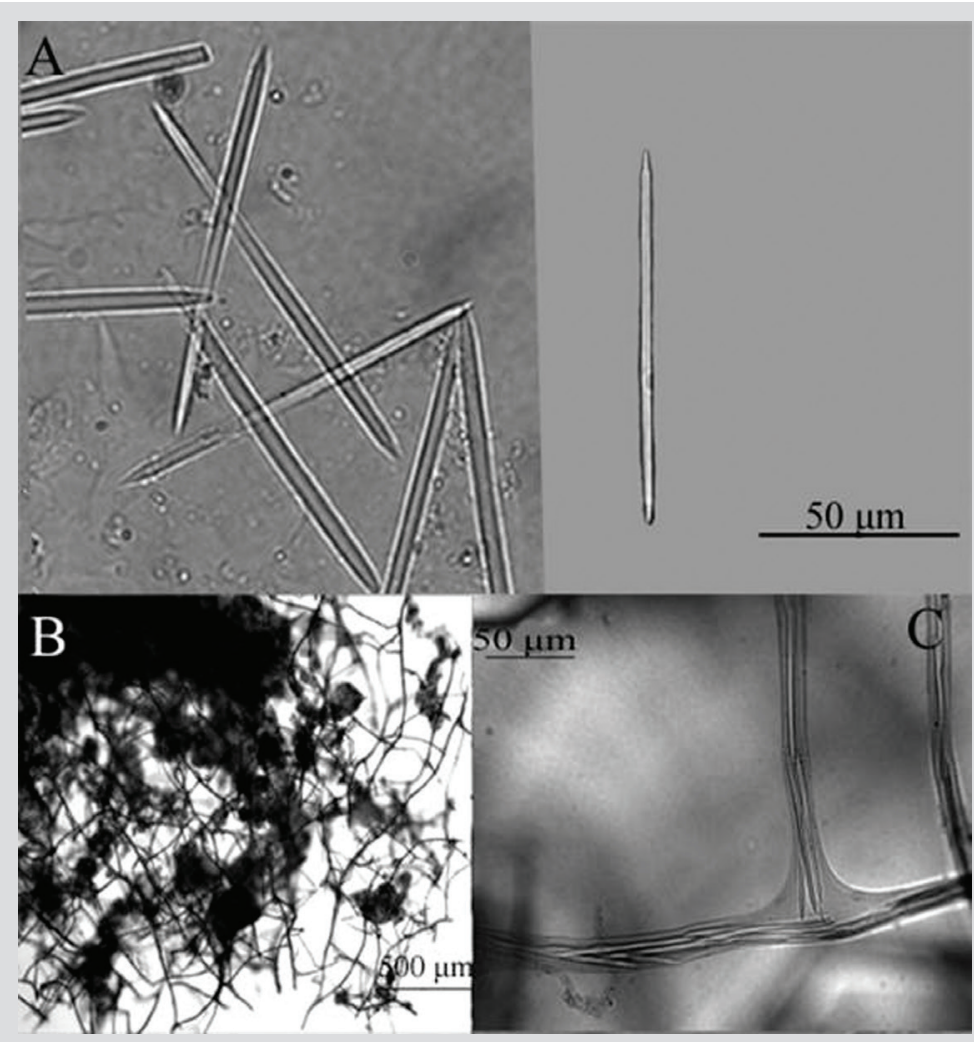

Figura 8. Chalinula nematifera. A) Oxeas. B) y C) Coanosoma.

Figure 8. Chalinula nematifera. A) Oxeas. B) and C) Choanosome.

Orden Poecilosclerida Topsent, 1928

Familia Crambeidae Lévi, 1963

\section{Especie 8. Crambe panamensis Maldonado, Cardona, Van Soest y Pomponi, 2001}

Figura 9; Lám. 1, fig. 6

Crambe panamensis Maldonado, Carmona, Van Soest y Pomponi, 2001:1264, fig. 1.

Material estudiado: INV POR1365, Punta Cruces, La Parguera-Piñas, 6 m, col. L. Chasqui, 16-08-2015.

Forma: revestimiento delgado de 15-20 cm de diámetro. Color: naranja-roja; blanco en alcohol.

Consistencia: dura, poco compresible. Ectosoma: tiloestilos y subtiloestilos erectos, a veces en mechones, sostienen el pinacodermo. Coanosoma: estrato basal de astroclonas, del que ascienden subtiloestilos y tiloestilos principales, convirtiéndose en mechones híspidos al llegar a la superficie (Figura 9H). Espículas: megascleras: tiloestilos ligeramente curvados con la cabeza bien definida, a veces irregular, 217-308-427 $\mu \mathrm{m}$ por 8,8-17,8-29,7 $\mu \mathrm{m}$, diámetro del tilo 10,1-16,5-27,6 $\mu \mathrm{m}$ (Figura 9C, 9F); subtiloestilos rectos y delgados con la cabeza ligeramente ovalada, 77-
Order Poecilosclerida Topsent, 1928

Family Crambeidae Lévi, 1963

\section{Species 8. Crambe panamensis Maldonado, Cardona, Van Soest and Pomponi, 2001}

Figure 9; Plate 1, fig. 6

Crambe panamensis Maldonado, Carmona, Van Soest and Pomponi, 2001: 1264, fig. 1.

Material studied: INV POR1365, Punta Cruces, La Parguera-Piñas, 6 m, col. L. Chasqui, 08-16-2015.

Shape: $15-20 \mathrm{~cm}$ diameter thin encrustation. Color: Redorange; white in alcohol.

Consistency: hard, not very compressible. Ectosome: tylostyles and subtylostyles, erect, sometimes tufted, support the pinacoderm. Choanosome: basal stratum of astroclones, from which subtylostyles and tylostyles ascend, turning into hispid tufts upon reaching the surface (Figure 9H). Spicules: megascleras: slightly curved tylostyles with well-defined head, sometimes irregular, 217-308-427 $\mu \mathrm{m}$ by $8.8-17.8-29.7 \mu \mathrm{m}$, tyle diameter 10.1-16.5-27.6 $\mu \mathrm{m}$ (Figure 9C, 9F); slender, straight subtylostyles with slightly oval heads, 77-185-232 $\mu \mathrm{m}$ by 2.4-4.0-5.8 $\mu \mathrm{m}$ (Figure 9B, 


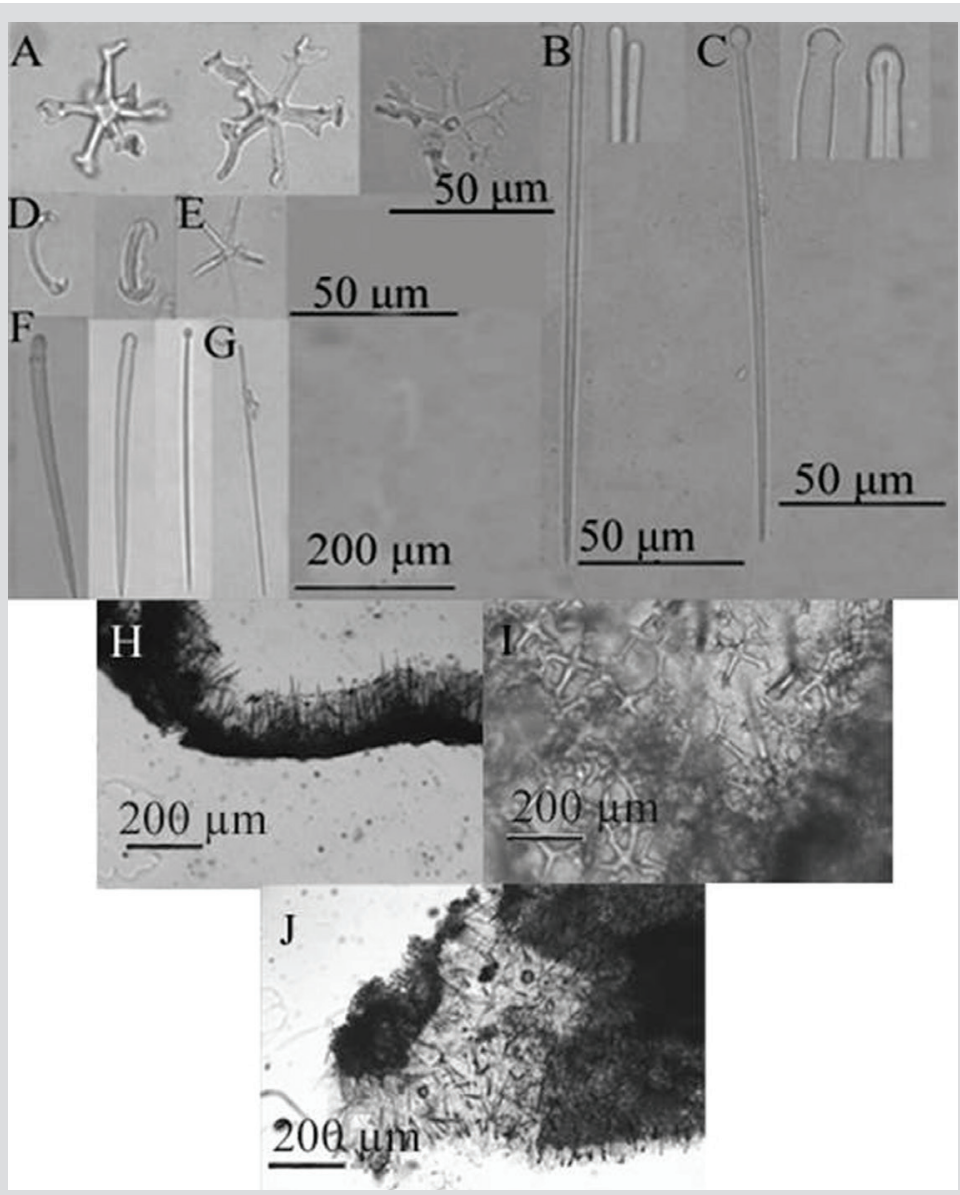

Figura 9. Crambe panamensis. A) Desmas astroclonas. B) Subtiloestilos delgados. C) Tiloestilos. D) Isochelas. E) Oxyáster. F) Tiloestilos. G) Subtiloestilo delgado. H) e I) Corte perpendicular a la superficie (arriba izq.) y vista de la costra basal de astroclonas (arriba der.). J) Corte parcialmente macerado que muestra parte del ectosoma (espículas verticales y mechones) y del coanosoma.
Figure 9. Crambe panamensis. A) Desmas astroclones. B) Thin subtylostyles. C) Tylostyles. D) Isochelae. E) Oxyaster. F) Tylostyles. G) Thin subtylostyle. H) and I) Section perpendicular to the surface (top left) and view of the basal astroclone crust (top right). J) Partially macerated section showing part of the ectosome (vertical spicules and tufts) and the choanosome.
185-232 $\mu \mathrm{m}$ por 2,4-4,0-5,8 $\mu \mathrm{m}$ (Figura 9B, 9G); desmas astroclonas, con un centro bien desarrollado, del cual irradian cuatro a seis actinas, 50-84-117 $\mu \mathrm{m}$ (diámetro total) (Figura 9A). Microscleras: oxiásteres, 4-5 radios, 9,9-21,1$29,8 \mu \mathrm{m}$ de diámetro (Figura 9E); isoquelas con tres dientes, 19,7-23,9-29,9 $\mu \mathrm{m}$ (Figura 9D).

Hábitat: sobre paredes verticales del arrecife rocoso.

Distribución: Pacífico de Panamá (Maldonado et al., 2001); Pacífico norte colombiano.

Comentarios: se confirmó el registro de Crambe panamensis por la presencia de desmas astroclonas junto con una categoría de tiloestilos coanosomales, subtiloestilos ectosomales pequeños y una categoría de isochelas (Maldonado et al., 2001). La localidad tipo es cercana al Pacífico norte colombiano.
9G); Desmas astroclones, with a well-developed center, from which four to six actins radiate, $50-84-117 \mu \mathrm{m}$ (total diameter) (Figure 9A). Microsclera: oxyasters, 4-5 radii, 9.9-21.1-29.8 $\mu \mathrm{m}$ in diameter (Figure 9E); isochelae with three teeth, 19.7-23.9-29.9 $\mu \mathrm{m}$ (Figure 9D).

Habitat: on vertical walls of the rocky reef.

Distribution: Pacific of Panama (Maldonado et al., 2001); Colombian North Pacific.

Comments: the record of Crambe panamensis was confirmed by the presence of astroclone desmas together with a category of choanosomeles tylostyles, small ectosoal subtylostyles, and a category of isochelae (Maldonado et al., 2001). The type locality is close to the Colombian North Pacific. 


\section{Especie 9. Discorhabdella littoralis Maldonado, Carmona, Van Soest y Pomponi, 2001}

Figura 10.

Discorhabdella littoralis Maldonado, Carmona, Van Soest y Pomponi, 2001: 1270, fig. 3.

Material estudiado: INV POR1401, cabo Marzo, La Foca, prof. $17 \mathrm{~m}$, col. L. Chasqui, 15-08-2015.

Forma: revestimiento delgado. Color: blanco en alcohol. Consistencia: muy delgada para notarlo; textura híspida al tacto. Ectosoma: espículas perforando el pinacodermo. Las sigmas se encuentran principalmente debajo de la superficie. Coanosoma: acantoestilos agrupados densamente en la base sobre una capa de espongina, de la que se proyectan hacia arriba los tiloestilos principales, que luego atraviesan la superficie de la esponja. Los subtilostilos secundarios están dispuestos en forma de ramas o mechones alrededor de los tiloestilos (Figura 10E). Espículas: megascleras: tiloestilos principales levemente curvados con la cabeza oval, 128-178-247 $\mu \mathrm{m}$ por 2,9-5,9-9,2 $\mu \mathrm{m}$ (Figura 10A); subtiloestilos secundarios, 118-144-171 $\mu \mathrm{m}$ por 3,2-5,37,2 $\mu \mathrm{m}$ (Figura 10B); acantoestilos, 20-30-42 $\mu \mathrm{m}$ por 4,1$6,1-8,7 \mu \mathrm{m}$ y con la cabeza grande, 9,2-12,3-18,1 $\mu \mathrm{m}$ de diámetro (Figura 10C). Microscleras: sigmas, 11,2-22,5$32,1 \mu \mathrm{m}$ (Figura 10D).

Hábitat: arrecifes rocosos someros.

Distribución: Pacífico de Panamá (Maldonado et al., 2001); Pacífico norte colombiano.

Comentarios: se comparó con las especies de Discorhabdella descritas por Maldonado et al. (2001) para la costa Pacífica de Panamá. Se definió como D. littoralis por la presencia de tiloestilos, subtiloestilos y sigmas.
Species 9. Discorhabdella littoralis

Maldonado, Carmona, Van Soest and

Pomponi, 2001

Figure 10.

Discorhabdella littoralis Maldonado, Carmona, Van Soest and Pomponi, 2001: 1270, fig. 3.

Material studied: INV POR1401, Cape Marzo, La Foca, prof. 17 m, col. L. Chasqui, 08-15-2015.

Shape: thin encrustation. Color: white in alcohol. Consistency: too thin to notice; hispid texture to the touch. Ectosome: spicules piercing the pinacoderm. Sigmas are found primarily below the surface. Choanosome: acanthostyles clustered densely at the base on a layer of spongin, from which the main tylostyles project upward, then traverse the surface of the sponge. The secondary subtylostyles are arranged in the shape of branches or tufts around the tylostyles (Figure 10E). Spicules: megascleras: slightly curved main tylostyles with oval head, 128178-247 $\mu \mathrm{m}$ by 2.9-5.9-9.2 $\mu \mathrm{m}$ (Figure 10A); secondary subtylostyles, 118-144-171 $\mu \mathrm{m}$ by 3.2-5.3-7.2 $\mu \mathrm{m}$ (Figure 10B); acanthostyles, 20-30-42 $\mu \mathrm{m}$ by 4.1-6.1-8.7 $\mu \mathrm{m}$ and with a large head, 9.2-12.3-18.1 $\mu \mathrm{m}$ in diameter (Figure 10C). Microscleres: sigmas, 11.2-22.5-32.1 $\mu \mathrm{m}$ (Figure 10D).

Habitat: shallow rocky reefs.

Distribution: Pacific of Panama (Maldonado et al., 2001); Colombian North Pacific.

Comments: it was compared with the Discorhabdella species described by Maldonado et al. (2001) for the Pacific coast of Panama. It was defined as D. littoralis by the presence of tylostyles, substylostyles, and sigma. 


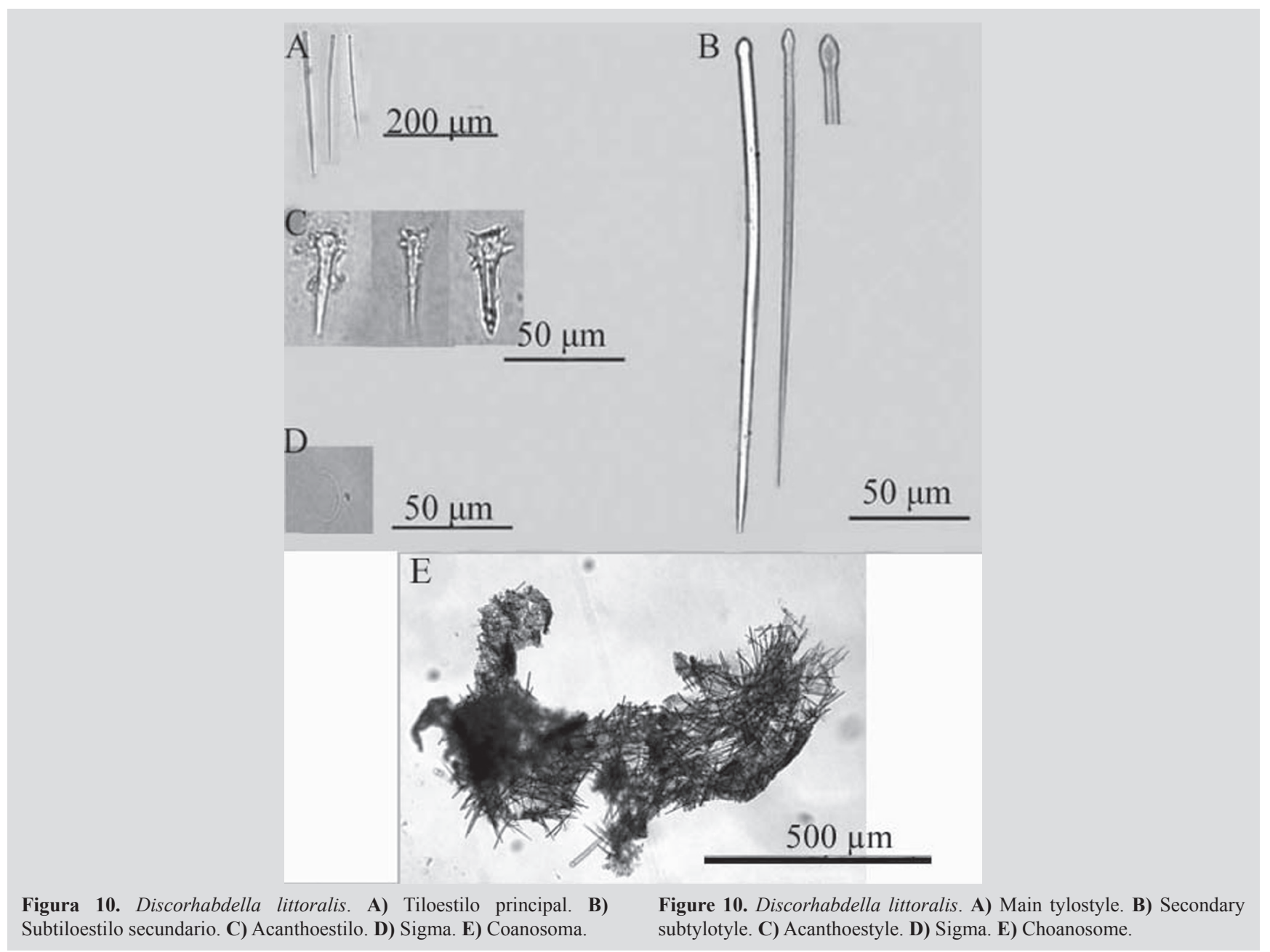

Familia Microcionidae Carter, 1875

\section{Especie 10. Clathria (Microciona) sp.}

Figura 11; Lám. 2, fig. 1

Material estudiado: INV POR1400, cabo Marzo, La Foca, $17 \mathrm{~m}$, col. L. Chasqui, 15-08-2015.

Forma: revestimiento delgado, ampliamente distribuido sobre el sustrato rocoso. Color: rojo escarlata, blanca en alcohol. Consistencia: frágil. Espículas: megascleras: estilos principales levemente curvados, ligeramente robustos en la base, cabeza con proyecciones espinosas y terminación en punta roma o afilada, 176-247-354 $\mu \mathrm{m}$ por 4,5-11,2-15,9 $\mu \mathrm{m}$ (Figura 11E); subtiloestilos delgados y rectos, 140-196-248 $\mu \mathrm{m}$ por 3,2-6,2-8,2 $\mu \mathrm{m}$ (Figura 11A); acantoestilos auxiliares rectos, con espinas a lo largo de toda la espícula, y la terminación de la punta gradualmente afilada, 68-93-120 $\mu \mathrm{m}$ por 3,8-5,8-8,0 $\mu \mathrm{m}$ (Figura 11B). Microscleras: isoquelas con ejes delgados, 16,5-19,1-30,0 $\mu \mathrm{m}$ (Figura 11D); toxas lisas en forma de V, 49-114-148 por 1,4-2,6-4,1 $\mu \mathrm{m}$ (Figura 11C).
Family Microcionidae Carter, 1875

\section{Species 10. Clathria (Microciona) sp.}

Figure 11; Plate 2, fig. 1

Material studied: INV POR1400, Cape Marzo, La Foca, 17 m, col. L. Chasqui, 08-15-2015.

Shape: thin encrustation, widely covering the rocky substratum. Color: scarlet red, white in alcohol. Consistency: fragile. Spicules: megascleres: main styles slightly curved, slightly robust at the base, head with spiny projections and ending in blunt or pointed tips, 176-247$354 \mu \mathrm{m}$ by $4.5-11.2-15.9 \mu \mathrm{m}$ (Figure 11E); thin and straight subtylostyles, 140-196-248 $\mu \mathrm{m}$ by 3.2-6.2-8.2 $\mu \mathrm{m}$ (Figure $11 \mathrm{~A})$; straight auxiliary acanthostyles, with spines along the entire spicule, and gradually tapered towards the tip, 6893-120 $\mu \mathrm{m}$ by 3.8-5.8-8.0 $\mu \mathrm{m}$ (Figure 11B). Microscleres: isochelae with a thin axis, 16.5-19.1-30.0 $\mu \mathrm{m}$ (Figure 11D); V-shaped smooth toxas, $49-114-148$ by $1.4-2.6-4.1 \mu \mathrm{m}$ (Figure 11C). 
Hábitat: en paredes del arrecife rocoso somero.

Distribución: Pacífico norte colombiano.

Comentarios: se tomó de referencia a Aguirre et al. (2011), quienes realizaron un cuadro comparativo de las especies del género Clathria Schmidt, 1862 del POT. La composición espicular es típica del subgénero Microciona Bowerbank, 1862, con estilos principales que en su mayoría tienen espinas solo en la cabeza, subtiloestilos auxiliares lisos, toxas lisas en una o dos categorías de longitud y con frecuencia de más de $100 \mu \mathrm{m}$ de longitud, e isoquelas en una sola categoría, normalmente menores de $15 \mu \mathrm{m}$ de largo. Se debe hacer un análisis más detallado y con material adicional para confirmar si se trata de una nueva especie.
Habitat: on shallow rocky reef walls.

Distribution: Colombian North Pacific.

Comments: reference was made to Aguirre et al. (2011), who made a comparative table of the species of the genus Clathria Schmidt, 1862 from the ETP. The spicular composition is typical of the subgenus Microciona Bowerbank, 1862, with main styles, mostly having spines only on the head, smooth auxiliary subtylostyles, smooth toxas in one or two length categories and often more than $100 \mu \mathrm{m}$ in length, and isochelae in a single category, usually less than $15 \mu \mathrm{m}$ long. More detailed analysis with additional material should be done to confirm if it is a new species.

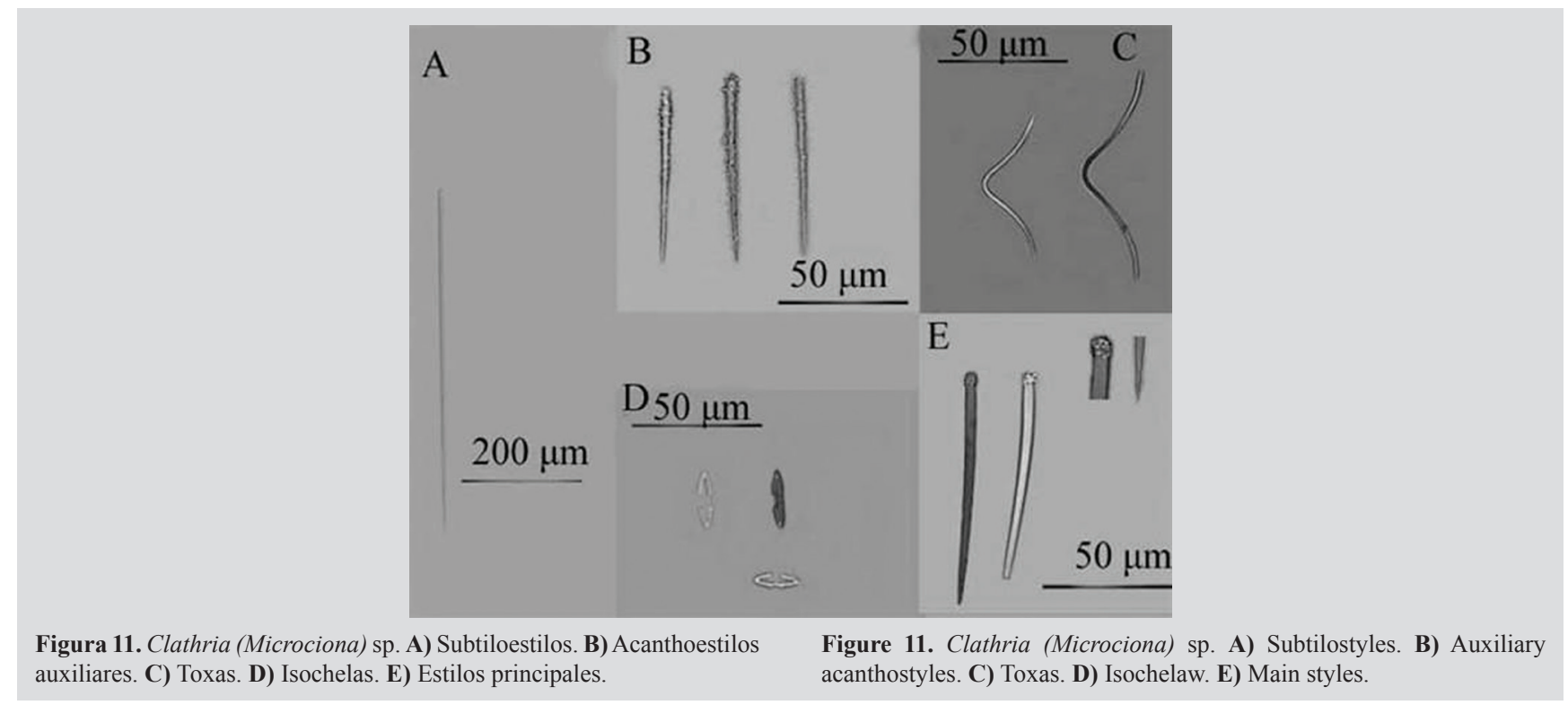

Orden Scopalinida Morrow y Cárdenas, 2015

Familia Scopalinidae Morrow, Picton, Erpenbeck, Boury-Esnault, Maggs y Allcock, 2012

Especie 11. Scopalina aff. ruetzleri (Wiedenmayer, 1977)

Figura 12.

?Ulosa ruetzleri Wiedenmayer, 1977: 145; Lámina 30, figs. 6-7.

Scopalina ruetzleri; Vega, 2012: 103, figs. 7.2.55. A, B.

Material estudiado: INV POR1378, golfo de Tribugá, Morromico, 12 m, col. L. Chasqui, 20-08-2015.

Forma: revestimiento delgado; superficie conulosa, con ósculos dispersos membranosos. Color: beige en alcohol.
Order Scopalinida Morrow and Cárdenas, 2015

Family Scopalinidae Morrow, Picton, Erpenbeck, Boury-Esnault, Maggs and Allcock, 2012

\section{Species 11. Scopalina aff. ruetzleri (Wiedenmayer, 1977)}

Figure 12.

?Ulosa ruetzleri Wiedenmayer, 1977: 145; Plate 30, figs. 6-7.

Scopalina ruetzleri; Vega, 2012: 103, figs. 7.2.55. A, B.

Material studied: INV POR1378, Gulf of Tribugá, Morromico, 12 m, col. L. Chasqui, 08-20-2015.

Shape: thin encrustation; conulose surface, with scattered membranous oscules. Color: beige in alcohol. Consistency: 
Consistencia: débil, blandamente compresible. Ectosoma: pinacodermo orgánico sostenido por los finales de los tractos ascendentes del coanosoma, que conforman los cónulos. Coanosoma: esqueleto de fibras multiespiculadas (4 a 7 espículas) ascendentes de 18-55 $\mu \mathrm{m}$ de grosor, que se ramifican hacia la superficie. Poca espongina recubre los tractos (Figura 12D). Espículas: estilos largos, ligeramente curvos de puntas simples, 299-552-761 $\mu \mathrm{m}$ por 4,1-6,9-11,6 $\mu \mathrm{m}$ (Figura 12C); anisoxeas (oxeas con un extremo más grueso y corto), 85-333$618 \mu \mathrm{m}$ por 3,0-6,5-11,6 $\mu \mathrm{m}$ (Figura 12B, 12C).

Hábitat: arrecifes rocosos someros.

Distribución: Pacífico mexicano (Vega, 2012); Pacífico norte colombiano.

Comentarios: las características del esqueleto y de las espículas son similares a Scopalina ruetzleri (Wiedenmayer, 1977) del Caribe (ver Zea, 1987); sin embargo, debe hacerse un análisis más detallado para determinar si se trata de la misma especie o de especies hermanas que no han divergido fenotípicamente lo suficiente desde que ocurrió asilamiento reproductivo por el levantamiento del istmo de Panamá, o si se trata de una colonización transístmica, para definir el uso de un nombre nuevo en los especímenes del Pacífico. weak, softly compressible. Ectosome: organic pinacoderm supported by the ends of the ascending tracts of the Choanosome, which make up the conules. Choanosome: skeleton of ascending multispicular fibers (4 to 7 spicules) 18-55 $\mu \mathrm{m}$ thick, branching towards the surface. Little spongin covers the tracts (Figure 12D). Spicules: long, slightly curved styles of simple tips, 299-552-761 $\mu \mathrm{m}$ by 4.1-6.9-11.6 $\mu \mathrm{m}$ (Figure 12C); anisoxeas (oxeas with a thicker and shorter end), 85-333-618 $\mu \mathrm{m}$ by 3.0-6.5-11.6 $\mu \mathrm{m}$ (Figure 12B, 12C).

Habitat: shallow rocky reefs.

Distribution: Mexican Pacific (Vega, 2012); Colombian North Pacific.

Comments: the skeletal and spicule characteristics are similar to Scopalina ruetzleri (Wiedenmayer, 1977) from the Caribbean (see Zea, 1987); however, a more detailed analysis should be made to determine if it is the same species or sister species that have not diverged phenotypically enough since reproductive isolation occurred due to the uprising of the isthmus of Panama, or if it is trans-isthmus colonization, to define the use of a new name for Pacific specimens.

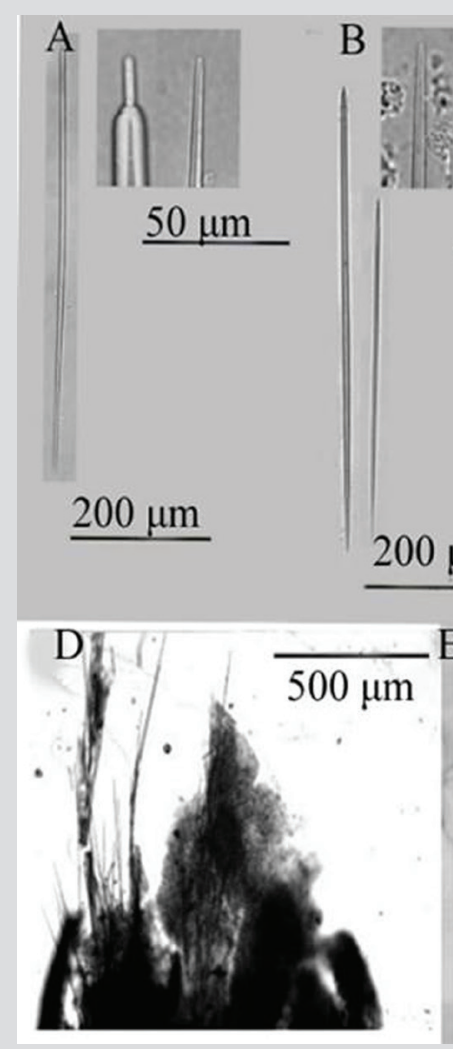

Figura 12. Scopalina aff. ruetzleri. A) y B) Anisoxeas. C) Estilos. D) y E) Tractos del coanosoma que atraviesan el ectosoma.
Figure 12. Scopalina aff. ruetzleri. A) and B) Anisoxeas. C) Styles. D) and $\mathbf{E}$ ) Tracts of the choanosome that cross the ectosome. 


\section{Especie 12. Scopalina sp. 2}

Figura 13; Lám. 2, fig. 2.

Material estudiado: INV POR1396, Punta Cruces, La Mina, sobre concha de gasterópodo, $15 \mathrm{~m}$, col. L. Chasqui, 16-08-2015. INV POR1408, cabo Marzo, Piedra de Rodrigo, sobre madera, 18.5 m, col. L. Chasqui, 15-08-2015.

Forma: revestimiento delgado extendiéndose aprox. 60 $\mathrm{mm}$ de manera irregular sobre el sustrato y sobre concha de gasterópodo, conuloso. Color: naranja; amarillo pálido en alcohol. Consistencia: suave, híspida al tacto. Ectosoma: pinacodermo orgánico; cónulos sostenidos por tractos espiculares erectos. Coanosoma: esqueleto de tractos multiespiculados ascendentes con 2 a 7 espículas en sección transversal, 34-91 $\mu \mathrm{m}$ de grosor, que se ramifican en la superficie; alta presencia de material foráneo (Figura 13C). Espículas: estilos delgados y largos, levemente curvos, 463-845-1265 $\mu \mathrm{m}$ por 4,3-6,5-8,9 $\mu \mathrm{m}$ (Figura 13A, 13B).

\section{Species 12. Scopalina sp. 2}

Figure 13; Plate 2, fig. 2.

Material studied: INV POR1396, Punta Cruces, La Mina, on gastropod shell, 15 m, col. L. Chasqui, 08-16-2015. INV POR1408, Cape Marzo, Piedra de Rodrigo, on wood, 18.5 m, col. L. Chasqui, 08-15-2015.

Shape: thin encrustation extending approx. $60 \mathrm{~mm}$ irregularly on the substratum and on a gastropod shell, conulose. Color: orange; pale yellow in alcohol. Consistency: soft, hispid to the touch. Ectosome: organic pinacoderm; conules supported by erect spicular tracts. Choanosome: skeleton of ascending multispiculate tracts with 2 to 7 spicules in cross-section, 34-91 $\mu \mathrm{m}$ thick, branching on the surface; high presence of foreign material (Figure 13C). Spicules: long, thin styles, slightly curved, 463-845-1265 $\mu \mathrm{m}$ by $4.3-$ 6.5-8.9 $\mu \mathrm{m}$ (Figure 13A, 13B).

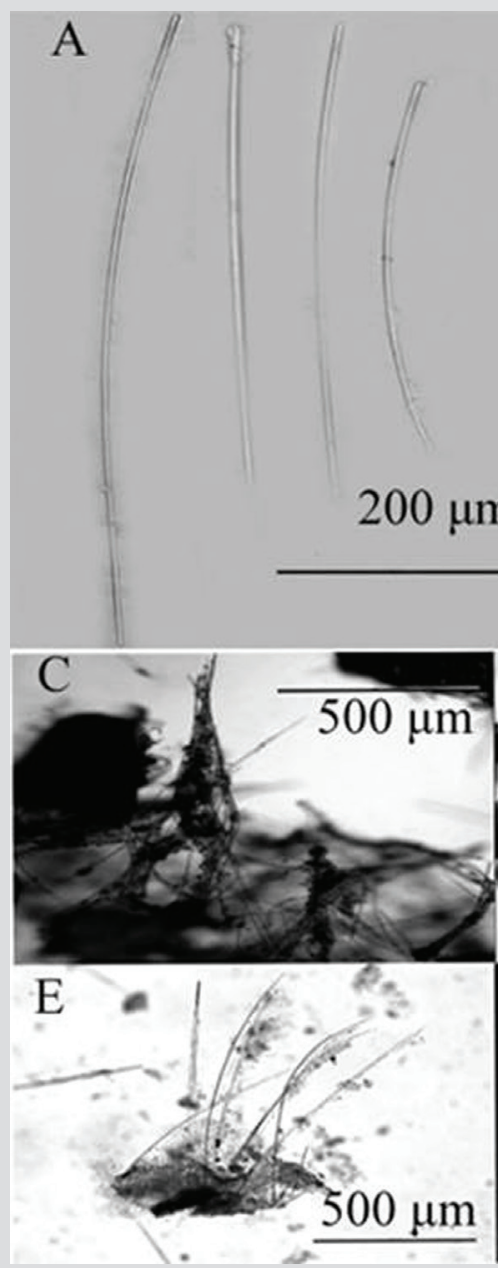

Figura 13. Scopalina sp. 2. A) Estilos. B) Cabezas de estilos. C), D), E) y F) Coanosoma.
B

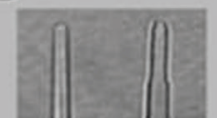

$50 \mu \mathrm{m}$
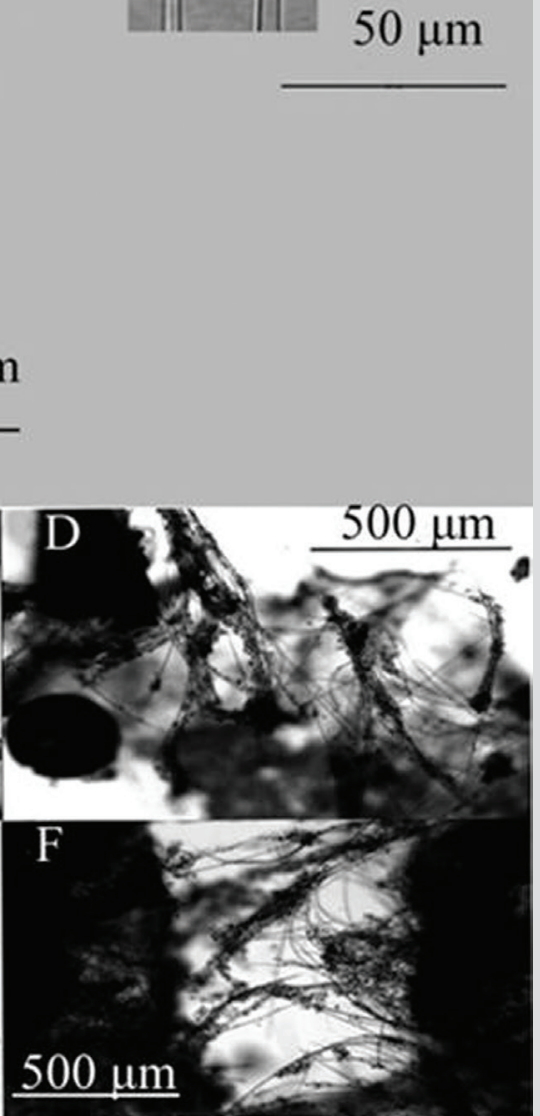

Figure 13. Scopalina sp. 2. A) Styles. B) Heads of styles. C), D), E) and F) Choanosome. 
Hábitat: sobre gasterópodo y madera en arrecife rocoso.

Distribución: Pacífico norte colombiano.

Comentarios: la ubicación genérica de este espécimen se realizó usando el Systema Porifera (Hooper et al., 2002). Exceptuando Scopalina aff. ruetzleri descrita aquí y en Vega (2012), no existen otras especies del género Scopalina registradas para el POT. Aunque tienen el mismo color, Scopalina sp. 2 es menos porosa y transparente, además de poseer solo estilos más elongados.

Orden Suberitida Chombard y Boury-Esnault, 1999

Familia Halichondriidae Gray, 1867

\section{Especie 13. Axinyssa isabela Carballo y Cruz-Barraza, 2008}

Figura 14; Lám. 2, fig. 3.

Axinyssa isabela Carballo y Cruz-Barraza, 2008: 60, figs. 2-5.

Material estudiado: INV POR1385, golfo de Tribugá, Morros de Jurubidá, 13,3 m, col. L. Chasqui, 20-08-2015. INV POR1397, cabo Marzo, Piedra de Eroito, 19 m, col. L. Chasqui, 15-08-2015.

Forma: masiva amorfa, con lóbulos bajos redondeados, extendiéndose irregularmente sobre el sustrato. Superficie lisa, ósculos de forma circular u ovalada de 1-4 mm de diámetro aprox. Color: amarillo oscuro, marrón a ocre en alcohol. Consistencia: firmemente compresible y carnosa en vida; preservada, se desmorona. Ectosoma: pinacodermo parcialmente desprendible, sin esqueleto especializado; tractos y espículas del coanosoma periférico lo atraviesan, pero también organizadas de forma entrecruzada o paratangencial. En la región subectosomal hay una capa de colágeno denso con espículas individuales o tractos. Coanosoma: tractos irregulares y plumosos, 48-94 $\mu \mathrm{m}$, ascienden y divergen hacia la superficie; muchas espículas en confusión (Figura 14C). Espículas: oxeas grandes y delgadas, ligeramente curvadas (Figura 14A), de puntas muy variables: afiladas, mucronadas, escalonadas y romas (Figura 14A, 14B), 526-647-765 $\mu \mathrm{m}$ por 5,6-10,6-16,1 $\mu \mathrm{m}$.

Hábitat: en paredes verticales del arrecife rocoso, cuevas y salientes.

Distribución: Pacífico mexicano (Carballo y Cruz-Barraza, 2008); Pacífico norte colombiano.

Comentarios: el profesor J.L. Carballo examinó las fotos tomadas en campo del espécimen in vivo y en el laboratorio del esqueleto y espículas y confirmó que se trata de esta especie por la forma de crecimiento y características morfológicas.
Habitat: on gastropod shells and on wood on rocky reefs.

Distribution: Colombian North Pacific.

Comments: the generic location of this specimen was made using the Systema Porifera (Hooper et al., 2002). Except for Scopalina aff. ruetzleri described here and in Vega (2012), there are no other species of the genus Scopalina recorded for the ETP. Although they have the same color, Scopalina sp. 2 is less porous and transparent, in addition to only having more elongated styles.

Order Suberitida Chombard and Boury-Esnault, 1999

Family Halichondriidae Gray, 1867

\section{Species 13. Axinyssa isabela Carballo and Cruz-Barraza, 2008}

Figure 14; Plate 2, fig. 3.

Axinyssa isabela Carballo and Cruz-Barraza, 2008: 60, figs. $2-5$.

Material studied: INV POR1385, Gulf of Tribugá, Morros de Jurubidá, 13.3 m, col. L. Chasqui, 08-20-2015. INV POR1397, Cape Marzo, Piedra de Eroito, 19 m, col. L. Chasqui, 08-15-2015.

Shape: massive amorphous, with rounded low lobes, spreading irregularly on the substratum. Smooth surface, round or oval shaped oscules of approx. 1-4 mm diameter. Color: dark yellow, brown to ocher in alcohol. Consistency: toughly compressible and fleshy, in life; when preserved, it crumbles. Ectosome: partially detachable pinacoderm, without specialized skeleton; tracts and spicules of the peripheral choanosome cross it, but are also organized in cross or paratangential fashion. In the subectosomal region, there is a dense collagen layer with individual spicules or tracts. Choanosome: irregular and plumose tracts, $48-94 \mu \mathrm{m}$, rise and diverge towards the surface; many spicules in confusion (Figure 14C). Spicules: large, thin oxeas, slightly curved (Figure 14A), with highly variable tips: sharp, mucronated, stepped, and blunt (Figure 14A, 14B), 526-647-765 $\mu \mathrm{m}$ by 5.6-10.6-16.1 $\mu \mathrm{m}$.

Habitat: on vertical rocky reef walls, caves, and ledges.

Distribution: Mexican Pacific (Carballo and Cruz-Barraza, 2008); Colombian North Pacific.

Comments: Professor J.L. Carballo examined the photos of the specimen taken in the field in vivo and of the skeleton and spicules in the laborataory and confirmed that it is this species by the shape of growth and morphological characteristics. 


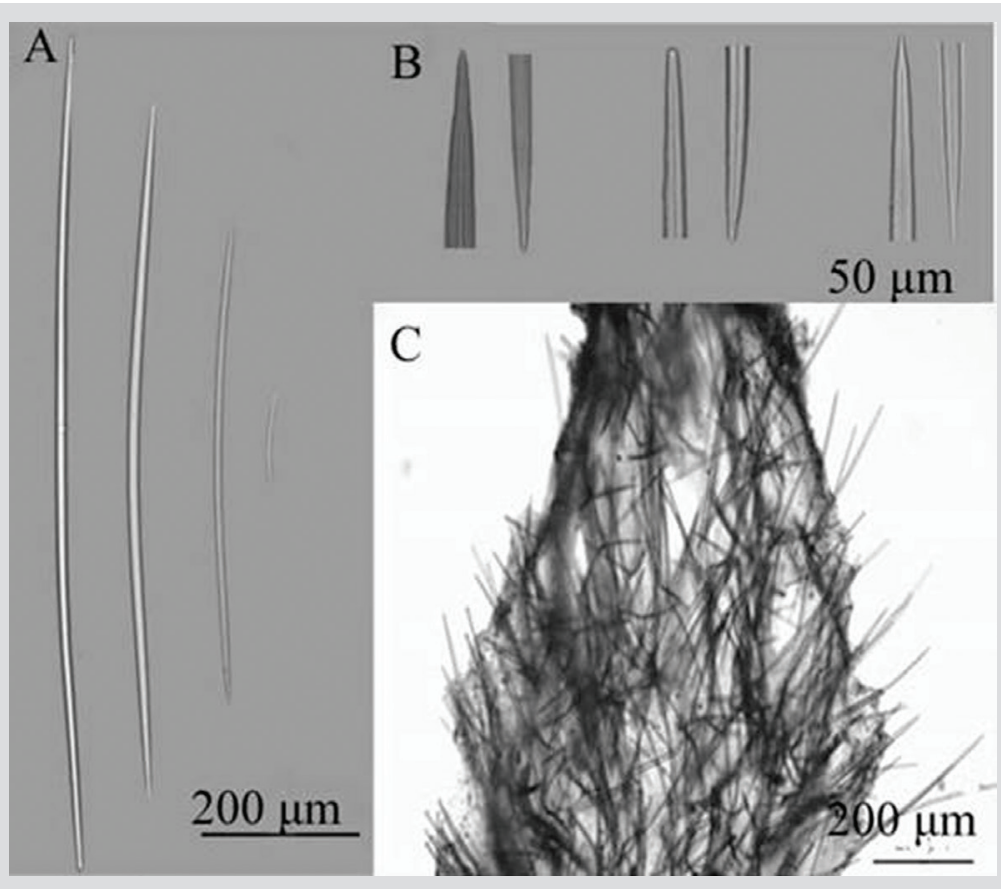

Figura 14. Axinyssa isabela. A) Oxeas. B) Extremos de oxeas. C) Corte perpendiculares a la superficie mostrando ectosoma y coanosoma.
Figure 14. Axinyssa isabela. A) Oxeas. B) Ends of oxeas. C) Section perpendicular to the surface showing ectosome and choanosome.
Especie 14. Epipolasis sp.

Figura 15.

Material estudiado: INV POR1387, golfo de Tribugá, Morros de Jurubidá, 10 m, col. L. Chasqui, 20-08-2015.

Forma: revestimiento delgado con superficie lisa, un poco corrugada de $6 \mathrm{~mm}$ de grosor aprox. Color: púrpura en alcohol, posiblemente por estar preservada junto a una Aplysina. Consistencia: compresible a desmoronadiza. Ectosoma: corteza de espículas más pequeñas y juntas que en el coanosoma. Coanosoma: esqueleto confuso de oxeas de varios tamaños (Figura 15C, 15D). Espículas: megascleras: oxeas ligeramente curvadas en el centro y frecuentemente dobladas en tres porciones, robustas, de puntas afiladas o romas, 149-195-290 $\mu \mathrm{m}$ por 6,4-10,4-16,9 $\mu \mathrm{m}$ (Figura 15A). Microscleras: rafidios rectos y delgados, 94-253-386 $\mu \mathrm{m}$ por 1,0-1,7-2,9 $\mu \mathrm{m}$ (Figura 15B).

Hábitat: sobre arrecifes rocosos someros.

Distribución: Pacífico norte colombiano.

Comentarios: la ubicación genérica se basó en el trabajo de Díaz et al. (1993), quienes hicieron una revisión de los géneros de Halichondriidae del Atlántico central. La diagnosis del género Epipolasis en De Laubenfels (1936a) concuerda con la del espécimen aquí descrito. Este es el primer registro del género en el POT.

\section{Species 14. Epipolasis sp.}

Figure 15.

Material studied: INV POR1387, Gulf of Tribugá, Morros de Jurubidá, 10 m, col. L. Chasqui, 08-20-2015.

Shape: thin encrustation with a smooth surface, slightly corrugated, approx 6mm thick. Color: purple in alcohol, possibly from being preserved alongside an Aplysina. Consistency: compressible to crumbly. Ectosome: cortex of spicules, smaller and closer together than in the choanosome. Choanosome: confused skeleton of oxeas of various sizes (Figure 15C, 15D). Spicules: megascleres: oxeas slightly curved in the center and frequently bent in three portions, robust, with pointed or blunt tips, 149-195$290 \mu \mathrm{m}$ by 6.4-10.4-16.9 $\mu \mathrm{m}$ (Figure 15A). Microscleres: straight and thin raphides, $94-253-386 \mu \mathrm{m}$ by 1.0-1.7-2.9 $\mu \mathrm{m}$ (Figure 15B).

Habitat: on shallow rocky reefs.

Distribution: Colombian North Pacific.

Comments: the generic location was based on the work of Díaz et al. (1993), who reviewed the genera of Halichondriidae from the central Atlantic. The diagnosis of the genus Epipolasis in De Laubenfels (1936a) agrees with that of the specimen described here. This is the first record of the genus in the ETP. 


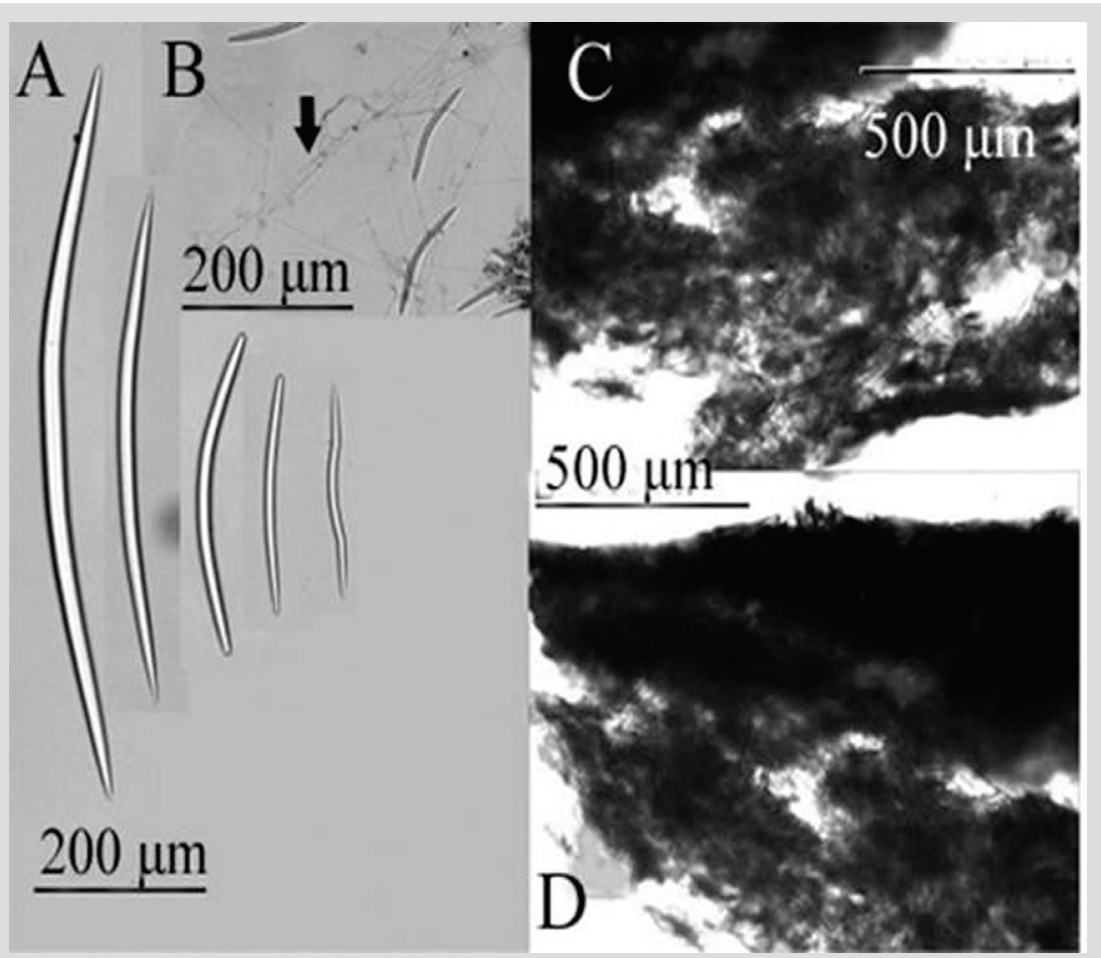

Figura 15. Epipolasis sp. A) Oxeas. B) Rafidios (señalados con flecha). C) y D) Esqueleto.

Figure 15. Epipolasis sp. A) Oxeas. B) Raphides (indicated with an arrow). C) and D) Skeleton.
Familia Suberitidae Schmidt, 1870

Especie 15. Terpios sp.

Figura 16; Lám. 2, fig. 4.

Material estudiado: INV POR1403, cabo Marzo, La Foca, sobre octocoral, 17 m, col. L. Chasqui, 15-08-2015.

Forma: revestimiento delgado liso. Color: amarillo brillante; amarillo pálido en alcohol. Consistencia: suave. Ectosoma: pinacodermo atravesado por mechones o hileras de espículas coanosomales, perpendiculares a la superficie. Coanosoma: esqueleto confuso con tractos o grupos de espículas sin dirección definida (Figura 16B, 16C). Espículas: tiloestilos cortos, ligeramente curvos, con cabeza irregular arrugada, abultada, estrecha, aplanada, o lobulada, 137-219-339 $\mu \mathrm{m}$ por 2,1-3,1-4,1 $\mu \mathrm{m}$ (Figura 16A).

Hábitat: sobre octocoral Carijoa riisei en arrecife rocoso.

Distribución: Pacífico norte colombiano.

Comentarios: no ha sido descrita ninguna esponja con tiloestilos de cabeza lobulada o irregular para el Pacífico Oriental, por lo que la identificación se realizó con base en Rützler y Smith (1993), quienes describieron nuevas especies del género Terpios en el Caribe. Se requiere de más material para definir su estatus taxonómico.

\section{Family Suberitidae Schmidt, 1870}

\section{Species 15. Terpios sp.}

Figure 16; Plate 2, fig. 4.

Material studied: INV POR1403, Cape Marzo, La Foca, octocoral envelope, 17 m, col. L. Chasqui, 08-15-2015.

Shape: smooth thin coating. Color: bright yellow; pale yellow in alcohol. Consistency: soft. Ectosome: pinacoderm crossed by tufts or rows of choanosomal spicules, perpendicular to the surface. Choanosome: confused skeleton with tracts or groups of spicules without definite direction (Figure 16B, 16C). Spicules: short tylostyles, slightly curved, with irregular wrinkled, bulging, narrow, flattened, or lobed head, 137-219-339 $\mu \mathrm{m}$ by 2.13.1-4.1 $\mu \mathrm{m}$ (Figure 16A).

Habitat: on octocoral Carijoa riisei on rocky reef.

Distribution: Colombian North Pacific.

Comments: no sponge with lobed or irregular tylostyle heads has been described for the Eastern Pacific, so the identification was made based on Rützler and Smith (1993), who described new species of the genus Terpios in the Caribbean. More material is required to define its taxonomic status. 


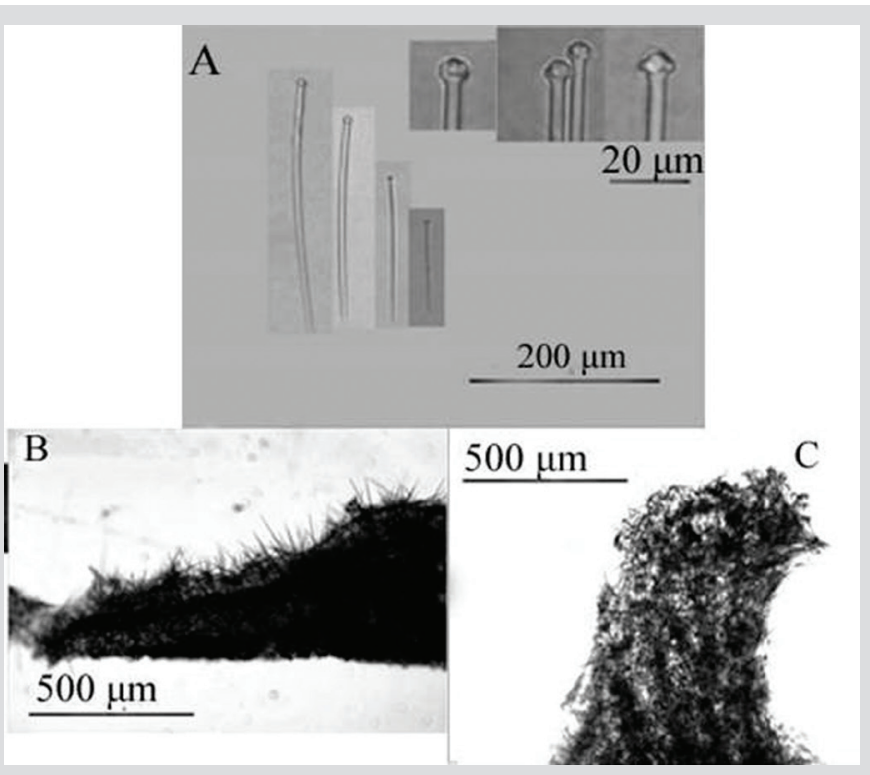

Figura 16. Terpios sp. A) Tiloestilos. B) y C) Cortes perpendiculares a la superficie mostrando ectosoma y coanosoma.
Figure 16. Terpios sp. A) Tylostyles. B) and C) Sections perpendicular to the surface showing ectosome and choanosome.
Orden Tetractinellida Marshall, 1876

Suborden Astrophorina Sollas, 1887

Familia Geodiidae Gray, 1867

Subfamilia Geodiinae Gray, 1867

\section{Especie 16. Geodia media Bowerbank, 1873}

Figura 17.

Geodia media Bowerbank, 1873: 13, Lám. II; Lendefeld, 1910, Lám. 16, figs. 1-21, Lám. 17, figs. 1-22; DesquerouxFaúndes y Van Soest, 1997: 408, figs. 69-76; Vega, 2012: 56, figs. 7.2.8. A, B, C.

Material estudiado: INV POR1363, Punta Cruces, La Parguera-Piñas, 6 m, col. L. Chasqui, 16-08-2015.

Forma: masiva amorfa, de pequeño tamaño, no más de 1-2 $\mathrm{cm}$ de diámetro y $0,9 \mathrm{~cm}$ de grosor. Superficie microrrugosa, llana. Color: blanca en alcohol. Consistencia: corteza dura, coanosoma frágil. Ectosoma: corteza de esterrásteres fuertemente agrupados, de 1576-1940 $\mu \mathrm{m}$ de grosor, conteniendo también oxiásteres y estrongilásteres.

Coanosoma: tractos de ortotriaenas y anatriaenas, 123-422 $\mu \mathrm{m}$ de ancho, separados entre sí 60-671 $\mu \mathrm{m}$, sosteniendo la corteza (Figura 17J). Espículas: megascleras: oxeas ligeramente curvas, 288-938-1445 $\mu \mathrm{m}$ por 10,2-21,3-32,7 $\mu \mathrm{m}$ (Figura 17E); estrongiloxeas con puntas desiguales, con terminaciones afiladas y romas, ligeramente curvas, 132-182-313 $\mu \mathrm{m}$ por 4,1-6,4-17,4 $\mu \mathrm{m}$ (Figura 17A); estilos robustos, poco frecuentes, 993-1049-1106 $\mu \mathrm{m}$ por 36,5-
Order Tetractinellida Marshall, 1876

Suborder Astrophorina Sollas, 1887

Family Geodiidae Gray, 1867

Subfamily Geodiinae Gray, 1867

Species 16. Geodia media Bowerbank, 1873

Figure 17.

Geodia media Bowerbank, 1873: 13, Plate II; Lendefeld, 1910, Plate 16, figs. 1-21, Plate 17, figs. 1-22; DesquerouxFaúndes and Van Soest, 1997: 408, figs. 69-76; Vega, 2012: 56, figs. 7.2.8. A, B, C.

Material studied: INV POR1363, Punta Cruces, La Parguera-Piñas, 6 m, col. L. Chasqui, 08-16-2015.

Shape: massive amorphous, small in size, no more than $1-2 \mathrm{~cm}$ in diameter and $0.9 \mathrm{~cm}$ thick. Micro-rough, even surface. Color: white in alcohol. Consistency: hard cortex, fragile choanosome. Ectosome: cortex of tightly packed sterrasters, 1576-1940 $\mu \mathrm{m}$ thick, also containing oxyasters and strongylasters. Choanosome: orthotriaene and anatriaene tracts, 123-422 $\mu \mathrm{m}$ wide, 60-671 $\mu \mathrm{m}$ apart, supporting the cortex (Figure 17J). Spicules: megascleres: slightly curved oxeas, 288-938-1445 $\mu \mathrm{m}$ by 10.2-21.3-32.7 $\mu \mathrm{m}$ (Figure 17E); strongyloxeas with uneven tips, with sharp and blunt ends, slightly curved, 132-182-313 $\mu \mathrm{m}$ by 4.1-6.4-17.4 $\mu \mathrm{m}$ (Figure 17A); robust styles, rare, 993-1049$1106 \mu \mathrm{m}$ by 36.5-38.9-41.2 $\mu \mathrm{m}$ (Figure 17C); orthotriaenes, rhabdome $512-1012-1497 \mu \mathrm{m}$ by 35-44-62 $\mu \mathrm{m}$, cladome 
38,9-41,2 $\mu \mathrm{m}$ (Figura 17C); ortotriaenas, rabdoma de 5121012-1497 $\mu \mathrm{m}$ por 35-44-62 $\mu \mathrm{m}$, cladoma $140-270-405 \mu \mathrm{m}$, clados 73-154-200 $\mu \mathrm{m}$ por 15,6-28,9-40,6 $\mu \mathrm{m}$ (Figura 17B); plagiotraenas, rabdoma $382-956-1301 \mu \mathrm{m}$ por $16,5-23,9$ $38,0 \mu \mathrm{m}$, cladoma 64-176 -314 $\mu \mathrm{m}$, clados 43-65-85 $\mu \mathrm{m}$ por 10,5-16,8-21,5 $\mu \mathrm{m}$ (Figura 17F); anatriaenas, rabdoma $382-727-879 \mu \mathrm{m}$ por $16,5-28,6-40,6 \mu \mathrm{m}$, cladoma $64-156-$ $246 \mu \mathrm{m}$, clados $22,6-26,2-30,0 \mu \mathrm{m}$ por $10,5-13,4-17,5 \mu \mathrm{m}$ (Figura 17D). Microscleras: esterrásteres, 37-68-91 $\mu \mathrm{m}$ por 40-55-70 $\mu \mathrm{m}$ (Figura 17G); estrongilásteres, 3,2-4,9-7,2 $\mu \mathrm{m}$ (Figura 17I); oxiásteres, 18,6-27,2-37,8 $\mu \mathrm{m}$ (Figura 17H).

Hábitat: en paredes de arrecifes rocosos someros.

Distribución: Costa Pacífica de México (Bowerbank, 1873; Vega, 2012); Panamá (Lendenfeld, 1910); islas Galápagos (Desqueyroux-Faúndez y Van Soest, 1997); Pacífico norte colombiano.

Comentarios: la identificación se basó en Lendenfeld (1910) y Desqueyroux-Faúndez y Van Soest (1997). La muestra es más similar a la que describe Lendenfeld (1910) por presentar estilos y orto-plagiotriaenas; sin embargo, las medidas difieren un poco de las de Colombia. La diferenciación entre especies de este género es bastante difícil, ya que se han descrito muchas hasta la actualidad.
140-270-405 $\mu \mathrm{m}$, clades 73-154-200 $\mu \mathrm{m}$ by 15.6-28.9$40.6 \mu \mathrm{m}$ (Figure 17B); plagiotraenes, rhabdome 382-956$1301 \mu \mathrm{m}$ by $16.5-23.9-38.0 \mu \mathrm{m}$, cladome $64-176-314 \mu \mathrm{m}$, clades $43-65-85 \mu \mathrm{m}$ by 10.5-16.8-21.5 $\mu \mathrm{m}$ (Figure 17F); anatriaenes, rhabdome $382-727-879 \mu \mathrm{m}$ by $16.5-28.6-40.6$ $\mu \mathrm{m}$, cladome $64-156-246 \mu \mathrm{m}$, clades $22.6-26.2-30.0 \mu \mathrm{m}$ by 10.5-13.4-17.5 $\mu \mathrm{m}$ (Figure 17D). Microscleres: sterrasters, 37-68-91 $\mu \mathrm{m}$ by $40-55-70 \mu \mathrm{m}$ (Figure $17 \mathrm{G}$ ); strongylasters, 3.2-4.9-7.2 $\mu \mathrm{m}$ (Figure 17I); oxyasters, 18.6-27.2-37.8 $\mu \mathrm{m}$ (Figure 17H).

Habitat: on shallow rocky reef walls.

Distribution: Pacific Coast of Mexico (Bowerbank, 1873; Vega, 2012); Panama (Lendenfeld, 1910); Galapagos Islands (Desqueyroux-Faúndez and Van Soest, 1997); Colombian North Pacific.

Comments: the identification was based on Lendenfeld (1910) and Desqueyroux-Faúndez and Van Soest (1997). The sample is more similar to that described by Lendenfeld (1910) for presenting styles and ortho-plagiotriaenes; however, the measurements differ somewhat from those of Colombia. The differentiation between species of this genus is quite difficult since many have been described to date.

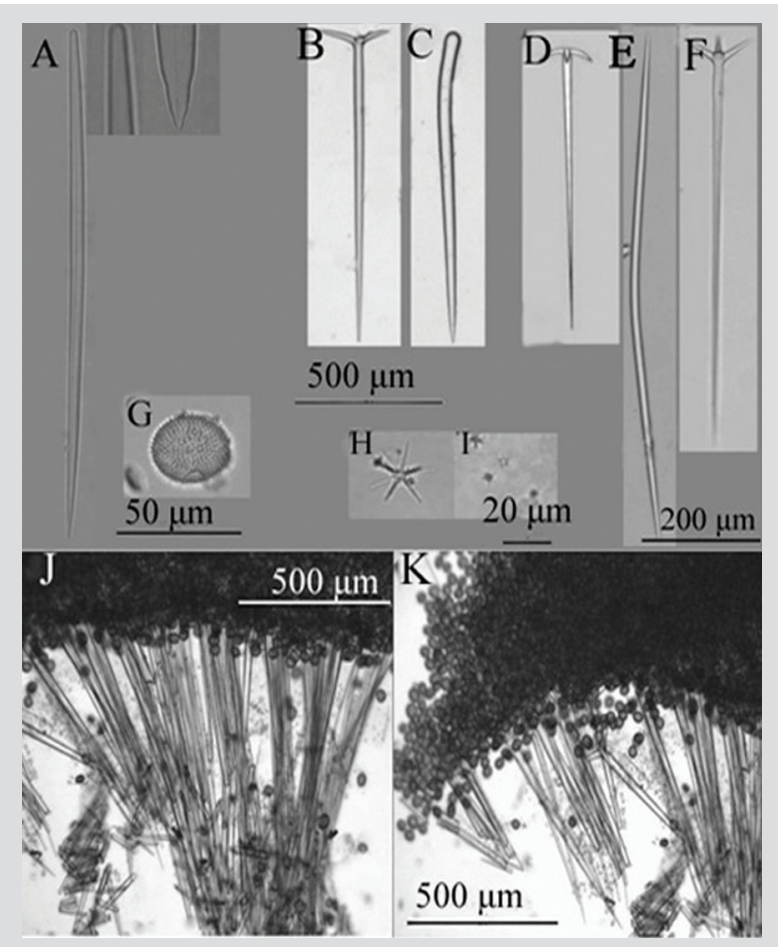

Figura 17. Geodia media. A) Estrongiloxea. B) Ortotriaena. C) Estilo. D) Anatriena. E) Oxea. F) Plagiotriaena. G) Esterráster. H) Oxiáster. I) Estrongilástere. J) y K) Corte perpendicular mostrando la corteza ectosómica y el coanosoma.
Figure 17. Geodia media. A) Strongyloxea. B) Ortotriaene. C) Style. D) Anatriena. E) Oxea. F) Plagiotriaena. G) Sterraster. H) Oxyaster. I) Strongylasters. J) and $\mathbf{K}$ ) Perpendicular section showing the ectosomal cortex and the choanosome. 
Subclase Keratosa Grant, 1861

Orden Dendroceratida Minchin, 1900

Familia Darwinellidae Merejkowsky, 1879

\section{Especie 17. Aplysilla sp.}

Figura 18; Lám. 2, fig. 5.

?Aplysilla sulphurea; Thiele 1905: 488, figs. 112, 114.

Non Aplysilla sulfurea Schulze, 1878 (especie válida del Mediterráneo y el Atlántico Oriental).

Non Aplysilla "sulfurea"; Escobar 2000: 101, fig. 39.

Non Aplysilla sulphurea; Vega, 2012: 113, figs. 7.2.65. A, B.

Material estudiado: INV POR1366, cabo Corrientes, Piedra de Jairo, 18 m, col. L. Chasqui, 18-08-2015. INV POR1367, cabo Corrientes, Piedra de Jairo, $18 \mathrm{~m}$, col. L. Chasqui, 1808-2015. INV POR1369, cabo Corrientes, Piedra de Colo, 14 m, col. L. Chasqui, 19-08-2015.

Forma: revestimiento de unos pocos $\mathrm{mm}$ de grosor; superficie conulosa. Color: amarillo pálido, beige en alcohol. Consistencia: blanda, fibras elásticas, pero algo templadas. Ectosoma: fibras de espongina sostienen el pinacodermo, pero pueden perforarlo y sobresalir más allá. Los cónulos en el material preservado tienen una altura de 118-342 $\mu \mathrm{m}$ (Figura 18C, 18D). Coanosoma: fibras de espongina individuales, erectas, de 35-89 $\mu \mathrm{m}$ de diámetro, que salen de una placa basal; algunas se ramifican y ascienden hacia la superficie (Figura 18A, 18B).

Hábitat: sobre arrecife rocoso junto con octocoral Carijoa riisei.

Distribución: Pacífico norte colombiano.

Comentarios: Aplysilla polyraphis De Laubenfels, 1930 registrada en California se distingue por ser púrpura oscura en vida y preservada, lo que difiere con las muestras chocoanas. Las fibras de espongina de Aplysilla lendenfeldi Thiele, 1905 de Chile son más gruesas, por lo que tampoco correspondería a esa especie. Las características de A. sulfurea, descrita para el estrecho de Magallanes (Thiele, 1905), son similares, pero debe hacerse un análisis más detallado para asegurar que se trata de la misma especie, especialmente por la gran distancia respecto al Pacífico colombiano. Ya que originalmente A. sulfurea se encuentra en el Atlántico oriental y el Mediterráneo, sería incorrecto llamar así al material del Pacífico Oriental. Escobar (2000) y Vega (2012) describen una Aplysilla sulphurea (sulphurea en el caso de Vega, 2012 y "sulfurea" en el caso de Escobar, 2000), que es amarilla en vida y se oxida a púrpura al ser fijada en alcohol, por lo que se infiere que tampoco se trataría de la misma especie.
Subclass Keratose Grant, 1861

Order Dendroceratida Minchin, 1900

Family Darwinellidae Merejkowsky, 1879

\section{Species 17. Aplysilla sp.}

Figure 18; Plate 2, fig. 5.

?Aplysilla sulphurea; Thiele 1905: 488, figs. 112, 114.

Non Aplysilla sulphurea Schulze, 1878 (Valid Mediterranean and Eastern Atlantic species).

Non Aplysilla "sulphurea”; Escobar 2000: 101, fig. 39.

Non Aplysilla sulphurea; Vega, 2012: 113, figs. 7.2.65. A, B.

Material studied: INV POR1366, Cape Corrientes, Piedra de Jairo, 18 m, col. L. Chasqui, 08-18-2015. INV POR1367, Cape Corrientes, Piedra de Jairo, 18 m, col. L. Chasqui, 0818-2015. INV POR1369, Cape Corrientes, Piedra de Colo, $14 \mathrm{~m}$, col. L. Chasqui, 08-19-2015.

Shape: encrustation of a few mm thick; conulouse surface. Color: pale yellow, beige in alcohol. Consistency: soft; elastic but somewhat rigid fibers. Ectosome: spongin fibers support the pinacoderm, but can pierce it and protrude beyond it. The conules in the preserved material have a height of 118-342 $\mu \mathrm{m}$ (Figure 18C, 18D). Choanosome: single, erect spongin fibers $35-89 \mu \mathrm{m}$ in diameter emerging from a basal plate; some branch out and rise to the surface (Figure 18A, 18B).

Habitat: over rocky reef along with octocoral Carijoa riisei.

Distribution: Colombian North Pacific.

Comments: Aplysilla polyraphis De Laubenfels, 1930 recorded in California is distinguished by being dark purple in life and preserved, which differs from the Chocó samples. The spongin fibers of Aplysilla lendenfeldi Thiele, 1905 from Chile are thicker, so it would not correspond to that species either. The characteristics of $A$. sulphurea, described for the Strait of Magellan (Thiele, 1905), are similar, but a more detailed analysis must be made to ensure that it is the same species, especially due to the great distance from the Colombian Pacific. Since A. sulphurea is originally found in the Eastern Atlantic and the Mediterranean, it would be incorrect to call the Eastern Pacific material that. Escobar (2000) and Vega (2012) describe an Aplysilla sulphurea (sulphurea in the case of Vega, 2012 and "sulfurea" in the case of Escobar, 2000), which is yellow in life and oxidizes to purple when fixed in alcohol, so it follows that it would not be the same species either. 


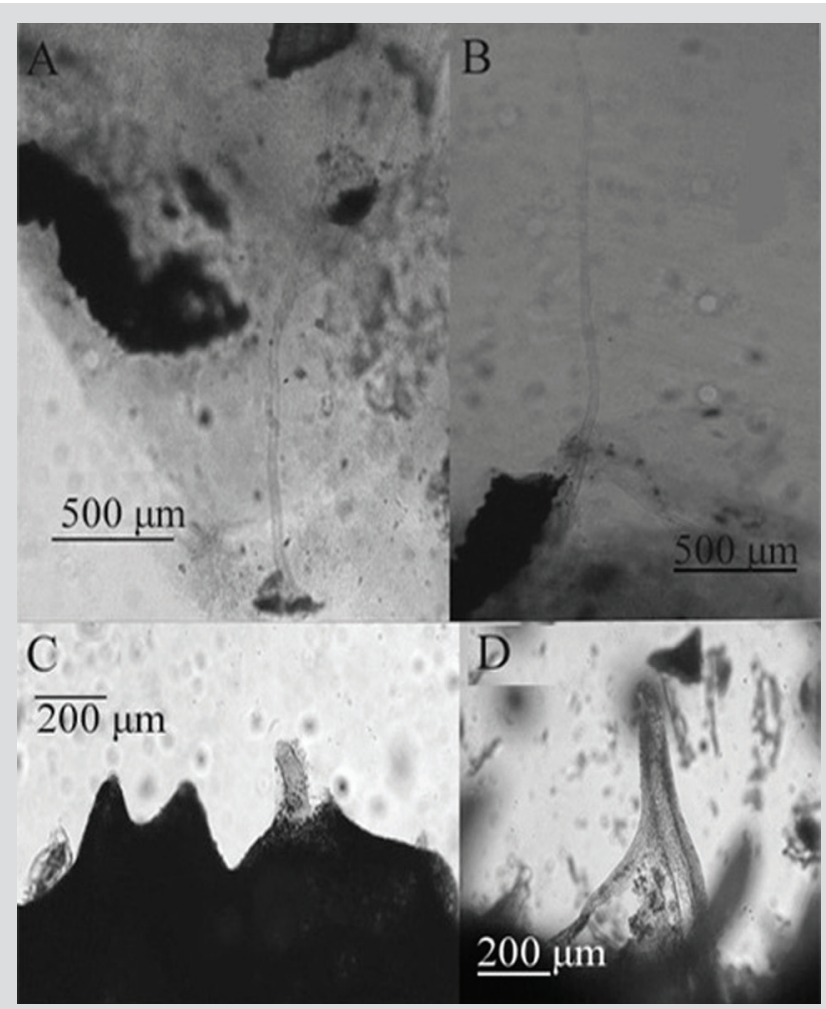

\section{Especie 18. Chelonaplysilla violacea (Lendenfeld, 1883) sensu Gómez, Carballo, Vázquez y Cruz, 2002}

Figura 19; Lám. 2, fig. 6.

?Aplysilla violacea Lendenfeld, 1883: 237, Lám. X, figs. 5, 7.

Chelonaplysilla violacea; Gómez, Carballo, Vázquez y Cruz, 2002: 234, fig. 7 (con sinonimia adicional); Vega, 2012: 114, figs. 7.2.66. A, B.

Material estudiado: INV POR1368, cabo Corrientes, Piedra de Colo, 19 m, col. L. Chasqui, 19-08-2015. INV POR1379, golfo de Tribugá, Morromico, fragmentos de roca, 11,9 m, col. L. Chasqui, 20-08-2015.

Forma: cojines delgados de algunos centímetros de diámetro, conulosos, con ósculos dispersos, ligeramente elevados con un collar orgánico. Color: púrpura grisáceo, con el collar oscular más oscuro; púrpura oscuro en alcohol. Consistencia: suave y frágil. Ectosoma: superficie conulosa reforzada por una red poligonal de detritus de 33-72 $\mu \mathrm{m}$ de grosor y ojo de malla de 50-164 $\mu \mathrm{m}$ (Figura 19A, 19B). Coanosoma: fibras solitarias de espongina de 37-80 $\mu \mathrm{m}$ de grosor (Figura 19C, 19D), que surgen de una placa basal y ascienden hacia la superficie; las fibras son meduladas y la médula ocupa 37-67 \% del diámetro.

\section{Species 18. Chelonaplysilla violacea \\ (Lendenfeld, 1883) sensu Gómez, Carballo, Vázquez and Cruz, 2002}

Figure 19; Plate 2, fig. 6.

?Aplysilla violacea Lendenfeld, 1883: 237, Plate X, figs. 5, 7. Chelonaplysilla violacea; Gómez, Carballo, Vázquez and Cruz, 2002: 234, fig. 7 (with additional synonymy); Vega, 2012: 114, figs. 7.2.66. A, B.

Material studied: INV POR1368, Cape Corrientes, Piedra de Colo, 19 m, col. L. Chasqui, 08-19-2015. INV POR1379, Gulf of Tribugá, Morromico, rock fragments, 11.9 m, col. L. Chasqui, 08-20-2015.

Shape: thin cushions a few centimeters in diameter, conular, with scattered oscules, slightly raised with an organic collar. Color: greyish purple, with darker dark collar; dark purple in alcohol. Consistency: soft and brittle. Ectosome: conulose surface reinforced by a 33-72 $\mu \mathrm{m}$ thick polygonal debris network and 50-164 $\mu \mathrm{m}$ mesh size (Figure 19A, 19B). Choanosome: solitary spongin fibers $37-80 \mu \mathrm{m}$ thick (Figure 19C, 19D), arising from a basal plate and rising to the surface; the fibers are pithed and the pith occupy $37-$ $67 \%$ of the diameter. 
Hábitat: sobre arrecife rocoso somero.

Distribución: costa Pacífica de México (Gómez et al., 2002); Pacífico norte colombiano.

Comentarios: el material se ajusta al que describen Gómez et al. (2002) de la costa Pacífica de México. Fue descrita originalmente del Pacífico Occidental, por lo que podría tratarse de un complejo de especies crípticas. De ahí la decisión de adscribirla solo al sentido de dichos autores. Es muy similar a Chelonaplysilla americana Van Soest, 2017 del Caribe, descrita para Colombia por Zea (1987) como C. erecta Row, 1911, lo que sugiere que serían especies hermanas.
Habitat: on a shallow rocky reef.

Distribution: Pacific coast of Mexico (Gómez et al., 2002); Colombian North Pacific.

Comments: the material conforms to that described by Gómez et al. (2002) from the Pacific coast of Mexico. It was originally described from the Western Pacific, so it could be a complex of cryptic species. Hence the decision to ascribe it only to the meaning of these authors. It is very similar to Chelonaplysilla americana Van Soest, 2017 from the Caribbean, described for Colombia by Zea (1987) as C. erecta Row, 1911, suggesting that they would be sister species.

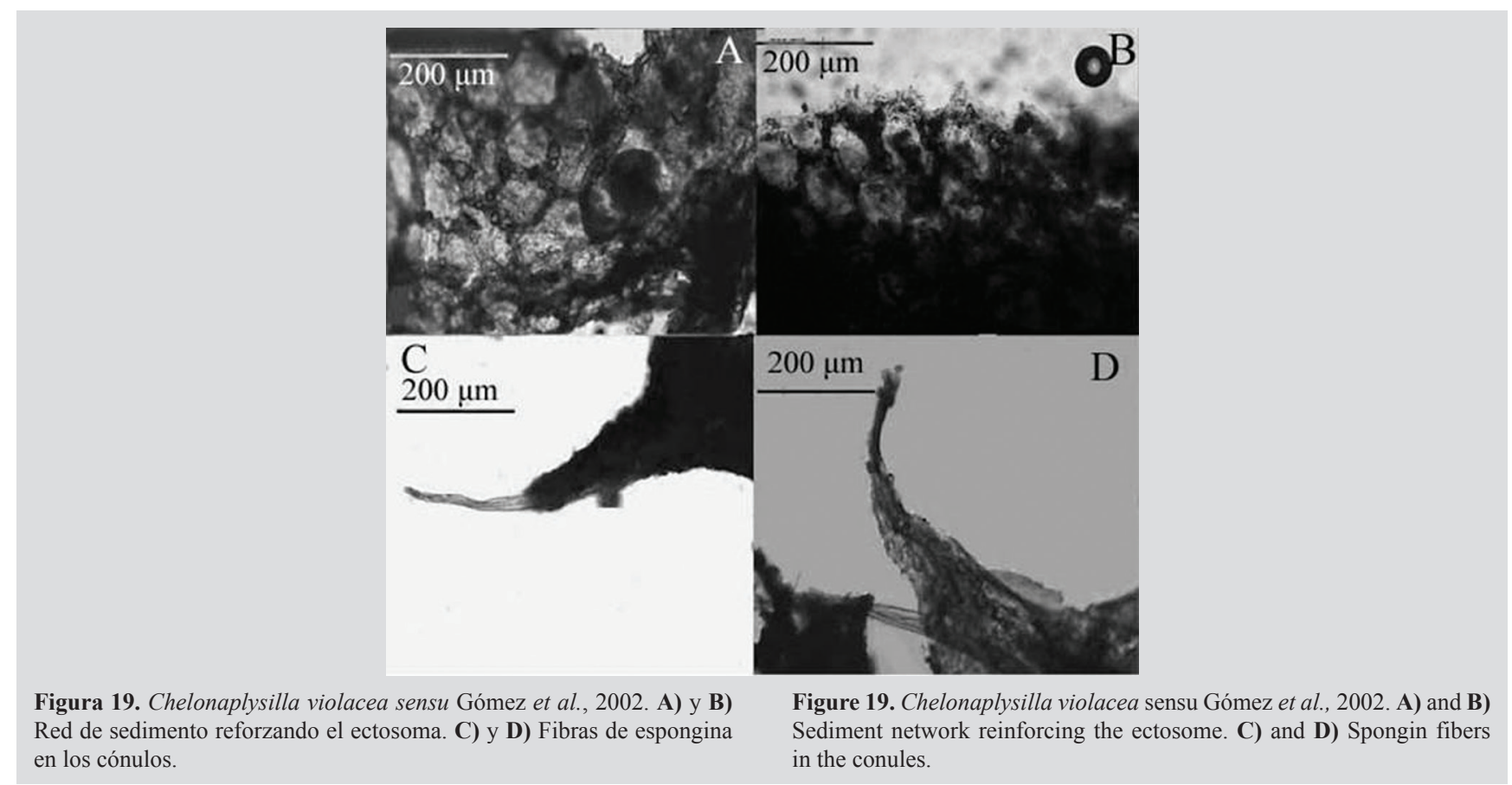

Orden Dictyoceratida Minchin, 1900

Familia Thorectidae Bergquist, 1978

Subfamilia Thorectinae Bergquist, 1978

Especie 19. Scalarispongia similis (Thiele, 1905)

Figura 20.

Cacospongia similis Thiele, 1905: 481, fig. 108; Desqueyroux-Faúndez y Van Soest, 1997: 455, figs. 200205.

Scalarispongia similis; Cook y Bergquist, 2000: 398 (transferencia de género)

Material estudiado: INV POR1392, Punta Cruces, La Viuda, 13-15 m, col. L. Chasqui, 14-08-2015.
Order Dictyoceratida Minchin, 1900

Family Thorectidae Bergquist, 1978

Subfamily Thorectinae Bergquist, 1978

Species 19. Scalarispongia similis (Thiele, 1905)

Figure 20.

Cacospongia similis Thiele, 1905: 481, fig. 108; Desqueyroux-Faúndez and Van Soest, 1997: 455, figs. 200205.

Scalarispongia similis; Cook and Bergquist, 2000: 398 (genus transfer).

Material studied: INV POR1392, Punta Cruces, La Viuda, 13-15 m, col. L. Chasqui, 08-14-2015. 
Forma: revestimiento delgado, con superficie conulosa. Color: rosado opaco en alcohol. Consistencia: firme, poco compresible. Ectosoma: pinacodermo grueso y fibroso; cónulos de 114-281 $\mu \mathrm{m}$ de altura, separados 314-1173 $\mu \mathrm{m}$, formados por fibras primarias que sobresalen del coanosoma. Coanosoma: red regular prismática de espongina con fibras primarias ascendentes, $20-80 \mu \mathrm{m}$ de grosor, rellenas con espículas foráneas y sedimento, separadas 143-373 $\mu \mathrm{m}$, interconectadas por fibras secundarias transversales, 25-7 $\mu \mathrm{m}$ de grosor, o una red secundaria con aberturas de 55-521 $\mu \mathrm{m}$ de diámetro (Figura 20).

Hábitat: sobre arrecife rocoso somero.

Distribución: islas Galápagos (Desqueyroux-Faúndez y Van Soest, 1997); islas Juan Fernández y Desventuradas de Chile (Thiele, 1905); hay un registro de la costa argentina (Burton, 1940) que requiere confirmación; Pacífico norte colombiano.

Comentarios: la organización en red rectangular y la presencia de espículas u otras partículas foráneas dentro de las fibras es diagnóstico de esta especie; el diámetro de las fibras es muy parecido al original, aunque las fibras secundarias son más gruesas en la muestra colombiana. Thiele (1905) la describió por primera vez en Chile, lo que puede indicar que esta especie se distribuye por todo el Pacífico Oriental. De confirmarse el registro de Burton (1940), se extendería también al sur del Atlántico americano.
Shape: thin encrustation, conulose surface. Color: dull pink in alcohol. Consistency: firm, little compressible. Ectosome: thick and fibrous pinacoderm; conules 114-281 $\mu \mathrm{m}$ high, separated $314-1173 \mu \mathrm{m}$, shaped by primary fibers protruding from the choanosome. Choanosome: Regular prismatic spongin network with ascending primary fibers, 20-80 $\mu \mathrm{m}$ thick, filled with foreign spicules and sediment, separated $143-373 \mu \mathrm{m}$, interconnected by transverse secondary fibers, $25-7 \mu \mathrm{m}$ thick, or a secondary network with meshes 55-521 $\mu \mathrm{m}$ in diameter (Figure 20).

Habitat: on a shallow rocky reef.

Distribution: Galapagos Islands (Desqueyroux-Faúndez and Van Soest, 1997); Juan Fernández and Desventuradas Islands of Chile (Thiele, 1905); there is a record from the Argentina coast (Burton, 1940) that requires confirmation; Colombian North Pacific.

Comments: the rectangular network organization and the presence of spicules or other foreign particles within the fibers are diagnostic of this species; the diameter of the fibers is very similar to the original, although the secondary fibers are thicker in the Colombian sample. Thiele (1905) described it for the first time in Chile, which may indicate that this species is distributed throughout the Eastern Pacific. If Burton's record (1940) is confirmed, it would also extend to the south of the American Atlantic.

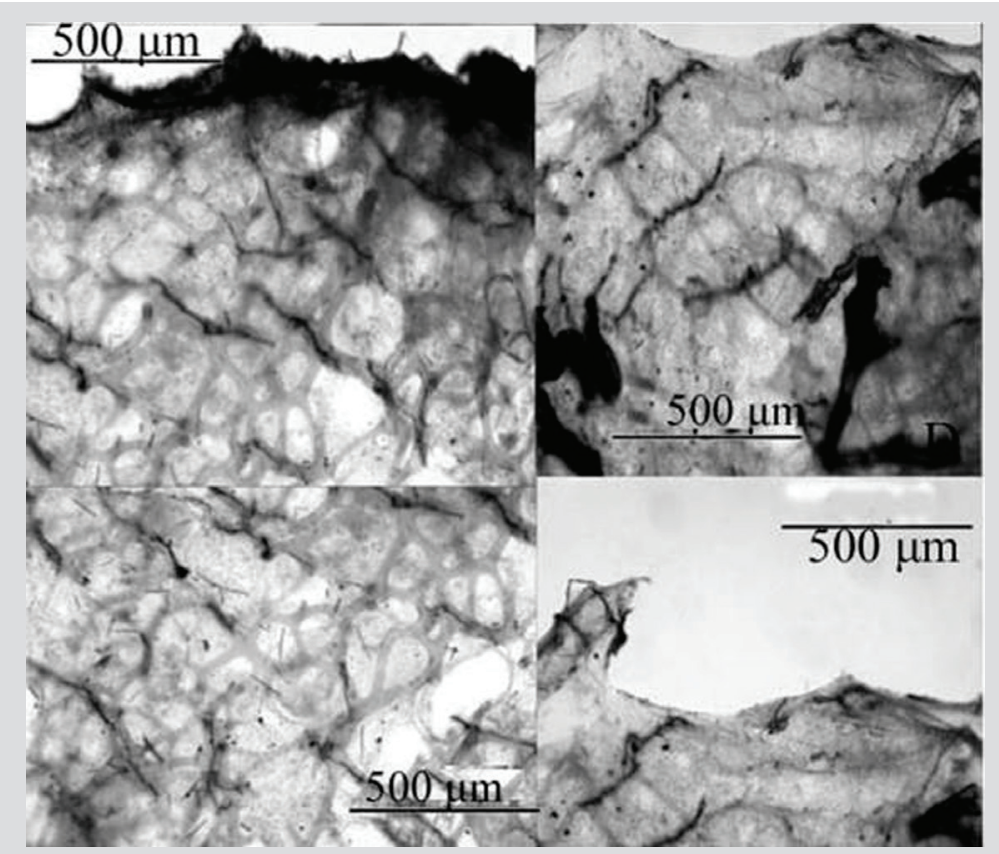

Figura 20. Scalarispongia similis. Cortes perpendiculares a la superficie mostrando el ectosoma y las redes de espongina del coanosoma.
Figure 20. Scalarispongia similis. Sections perpendicular to the surface showing the ectosome and the spongin networks of the choanosome. 
Subclase Verongimorpha Erpenbeck, Sutcliffe, De Cook, Dietzel, Maldonado, Van Soest, Hooper y Wörheide, 2012

Orden Chondrosiida Boury-Esnault y Lopes, 1985

Familia Chondrosiidae Schulze, 1877

\section{Especie 20. Chondrosia tenochca Carballo, Gómez, Cruz-Barraza y Flores-Sánchez, 2003}

Figura 21; Lám. 3, fig. 1.

Chondrosia tenochca Carballo, Gómez, Cruz-Barraza y Flores-Sánchez, 2003: 517; Vega, 2012: 87, figs. 7.2.39. A, $\mathrm{B}, \mathrm{C}$.

Material estudiado: INV POR1371, cabo Corrientes, Piedra de Colo, 11 m, col. L. Chasqui, 19-08-2015.

Forma: revestimiento de 1-2 mm de grueso, estirado sobre el sustrato y parcialmente fragmentado; superficie lisa, llana, brillante. Color: púrpura oscuro a negro, beige en la superfície y púrpura grisáceo en el interior en alcohol. Consistencia: tenaz, correosa. Ectosoma: sin esqueleto. En cortes se observa una corteza orgánica de unos 300 $\mu \mathrm{m}$ de grosor compuesta de dos capas, la más superficial más clara y la interna más densa, producto aparente de células granulosas. Coanosoma: muy denso, fibroso, pero menos granuloso que el ectosoma, sin esqueleto de fibras de espongina o espículas; se observan algunos canales que desembocan en los ósculos (Figura 21).

Hábitat: sobre arrecife rocoso somero.

Distribución: costa Pacífica de México (Carballo et al., 2003; Vega, 2012); Pacífico norte colombiano.

Comentarios: inicialmente se creía que se trataba de una ascidia colonial, pero se descartó al evidenciar una corteza densa de células que va disminuyendo hacia la parte basal y algunos canales carnosos en el coanosoma. Hasta el momento, este es el segundo registro de esta especie para el POT.
Subclass Verongimorpha Erpenbeck, Sutcliffe, De Cook, Dietzel, Maldonado, Van Soest, Hooper and Wörheide, 2012

Order Chondrosiida Boury-Esnault and Lopes, 1985 Family Chondrosiidae Schulze, 1877

\section{Species 20. Chondrosia tenochca Carballo, Gómez, Cruz-Barraza and Flores-Sánchez, 2003}

Figure 21; Plate 3, fig. 1.

Chondrosia tenochca Carballo, Gómez, Cruz-Barraza and Flores-Sánchez, 2003: 517; Vega, 2012: 87, figs. 7.2.39.A, $\mathrm{B}, \mathrm{C}$.

Material studied: INV POR1371, Cape Corrientes, Piedra de Colo, 11 m, col. L. Chasqui, 08-19-2015.

Shape: encrustation 1-2 mm thick, stretched over the substratum and partially fragmented; smooth, flat, shiny surface. Color: dark purple to black, beige on the surface, and grayish-purple on the inside in alcohol. Consistency: tough, leathery. Ectosome: without a skeleton. In sections, an organic crust of about $300 \mu \mathrm{m}$ thick is observed, composed of two layers, the clearest superficial one and the inner denser one, an apparent product of granular cells. Choanosome: very dense, fibrous, but less granular than the ectosome, without skeleton of spongin fibers or spicules; some canals that flow into the oscules are observed (Figure 21).

Habitat: on a shallow rocky reef.

Distribution: Pacific coast of Mexico (Carballo et al., 2003; Vega, 2012); Colombian North Pacific.

Comments: initially it was believed that it was a colonial ascidian, but it was discarded when a dense cortex of cells that decreases towards the basal part was evidenced, with some fleshy channels in the choanosome. So far, this is the second record of this species for the ETP. 

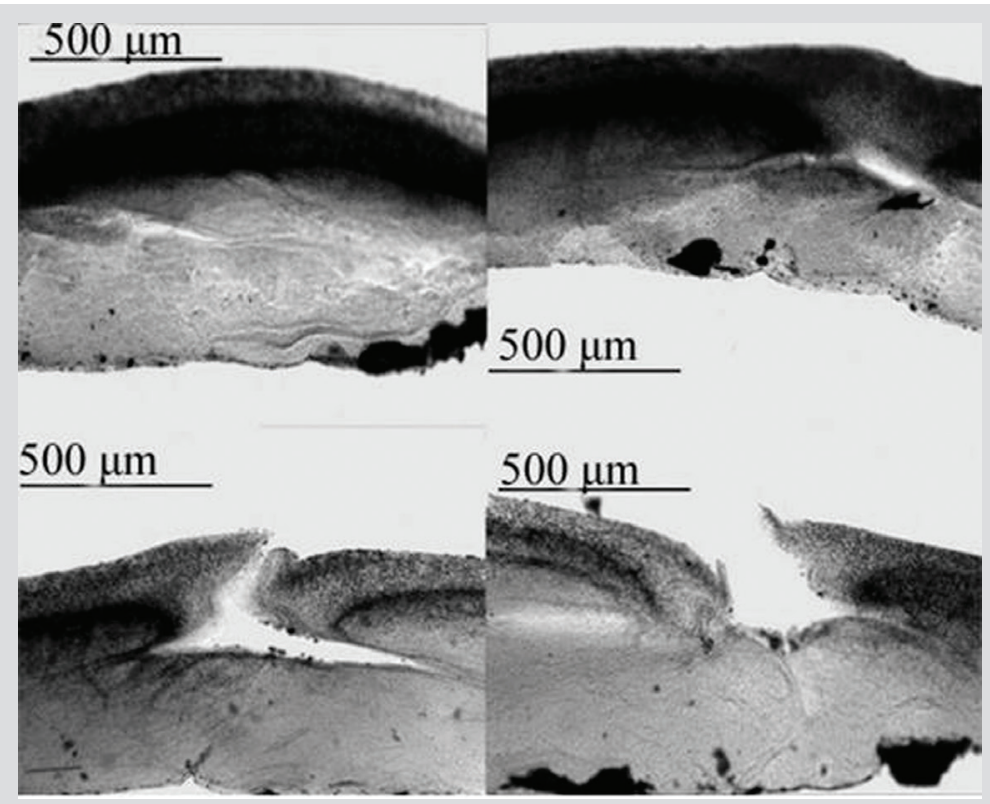

Figura 21. Chondrosia tenochca. Cortes perpendiculares mostrando el ectosoma y el coanosoma, con canales y ósculos.
Figure 21. Chondrosia tenochca. Perpendicular sections showing the ectosome and choanosome, with canals and oscules.
Orden Verongiida Bergquist, 1978

Familia Aplysinidae Carter, 1875

\section{Especie 21. Aplysina chiriquiensis Díaz, Van Soest, Rützler y Guzmán, 2005}

Figura 22; Lám. 3, fig. 2.

Aplysina chiriquiensis Díaz, Van Soest, Rützler y Guzmán, 2005: 3, figs. 1-3.

Material estudiado: INV POR1402, cabo Marzo, La Foca, 18,3 m, col. L. Chasqui, 15-08-2015. INV POR1405, cabo Marzo, Piedra de Rodrigo, 18,5 m, col. L. Chasqui, 15-08-2015.

Forma: erecta, de ramas cilíndricas engrosadas o lateralmente comprimidas con hinchazones anulares, proyecciones en forma de dedos o redondeadas y extremos nudosos que salen del tallo principal. Altura entre 10 y $45 \mathrm{~cm}$; ramas 3-12 $\mathrm{mm}$ de grosor y 21-71 mm de largo. Ósculos circulares, distribuidos en una o más filas a lo largo de las ramas, con una membrana tipo collar. Color: exterior amarillo ocre, interior amarillo intenso; púrpura oscuro en alcohol. Consistencia: ligeramente compresible y elástica. Ectosoma: superficie microconulosa; cónulos distribuidos regularmente, formados por los finales en crecimiento de las fibras del coanosoma, que sostienen el pinacodermo. Coanosoma: esqueleto como una red poligonal u oval de fibras color ámbar (Figura 22C, 22D), laminadas concéntricamente, 49-117 $\mu \mathrm{m}$ de grosor, formando mallas de 135-535 $\mu \mathrm{m}$ de diámetro. Las fibras tienen una
Order Verongiida Bergquist, 1978

Family Aplysinidae Carter, 1875

\section{Species 21. Aplysina chiriquiensis Díaz, Van Soest, Rützler and Guzmán, 2005}

Figure 22; Plate 3, fig. 2.

Aplysina chiriquiensis Díaz, Van Soest, Rützler and Guzmán, 2005: 3, figs. 1-3.

Material studied: INV POR1402, Cape Marzo, La Foca, 18.3 m, col. L. Chasqui, 08-15-2015. INV POR1405, Cape Marzo, Piedra de Rodrigo, 18.5 m, col. L. Chasqui, 0815-2015.

Shape: erect, thickened, or laterally compressed cylindrical branches with annular swellings, finger-shaped or rounded projections, and knotty ends emerging from the main stem. Height between 10 and $45 \mathrm{~cm}$; branches 3-12 mm thick and 21-71 mm long. Circular oscules, distributed in one or more rows along the branches, with a collar-like membrane. Color: ocher yellow exterior, intense yellow interior; dark purple in alcohol. Consistency: slightly compressible and elastic. Ectosome: microconulous surface; conules regularly distributed, shaped by the growing ends of the fibers of the choanosome, which support the pinacoderm. Choanosome: skeleton as a polygonal or oval network of amber fibers (Figure 22C, 22D), concentrically laminated, 49-117 $\mu \mathrm{m}$ thick, forming meshes of 135-535 $\mu \mathrm{m}$ in diameter. The fibers have a dark granular pith that occupies $31-57 \%$ of 
médula granular oscura que ocupa $31-57 \%$ del diámetro. Hacia la superficie, las fibras tienden a ser dendríticas, con un largo de 189-584 $\mu \mathrm{m}$ (Figura 22A, 22B), más delgadas que las ubicadas hacia el tallo principal.

Hábitat: abundante en los arrecifes rocosos someros.

Distribución: Costa Rica, Panamá, islas Galápagos (Díaz et al., 2005); Pacífico norte colombiano.

Comentarios: se registra por primera vez para el Pacífico norte colombiano y para el Pacífico colombiano en general. Es la única especie del género con forma de ramas verticales en el POT. the diameter. Towards the surface, the fibers tend to be dendritic, with a length of 189-584 $\mu \mathrm{m}$ (Figure 22A, 22B), thinner than those located towards the main stem.

Habitat: abundant on shallow rocky reefs.

Distribution: Costa Rica, Panama, Galapagos Islands (Díaz et al., 2005); Colombian North Pacific.

Comments: it is recorded for the first time for the Colombian North Pacific and the Colombian Pacific in general. It is the only species of the genus with the shape of vertical branches in the ETP.

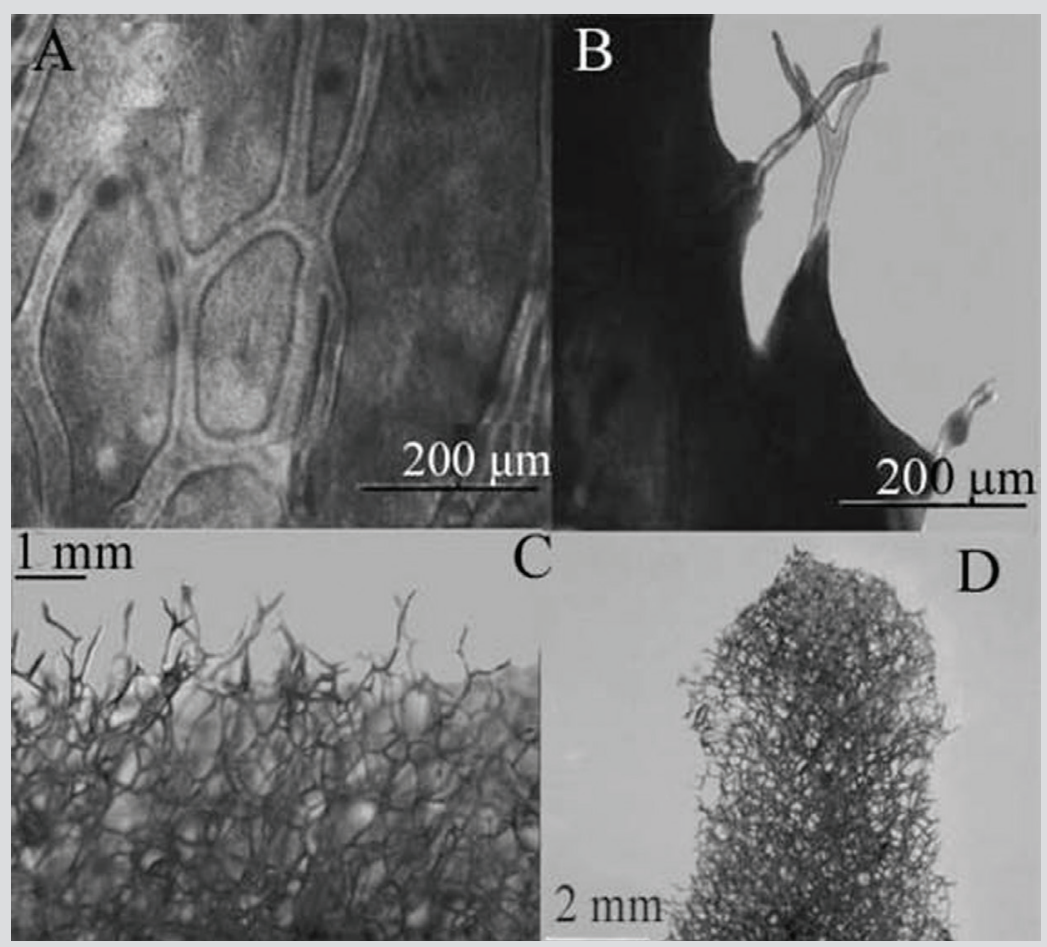

Figura 22. Aplysina chiriquiensis. A) y B) Corte histológico de esqueleto. C) y D) Retículo esquelético tridimensional (tejido eliminado en baño de hipoclorito).
Figure 22. Aplysina chiriquiensis. A) and B) Histological section of the skeleton. C) and D) Three-dimensional skeletal reticulum (tissue removed in a bleach bath)

\section{Especie 22. Aplysina gerardogreeni Gómez y Bakus, 1992}

Figura 23; Lám. 3, fig. 3.

Aplysina gerardogreeni Gómez y Bakus, 1992: 176, Lám. 1-2; Cruz-Barraza, Carballo, Rocha-Olivares, Ehrlich y Hog, 2012: 4, figs. 1E, F; 2I, J; Vega, 2012: 116, figs. 7.2.68. A, B.

Suberea gerardogreeni; Escobar, 2000: 108, figs. 41-42.

Material estudiado: INV POR1374, golfo de Tribugá, Morromico, 11,7 m, col. L. Chasqui, 20-08-2015. INV

\section{Species 22. Aplysina gerardogreeni Gómez and Bakus, 1992}

Figure 23; Plate 3, fig. 3.

Aplysina gerardogreeni Gómez and Bakus, 1992: 176, Plate 1-2; Cruz-Barraza, Carballo, Rocha-Olivares, Ehrlich and Hog, 2012: 4, figs. 1E, F; 2I, J; Vega, 2012: 116, figs. 7.2.68. A, B.

Suberea gerardogreeni; Escobar, 2000: 108, figs. 41-42.

Material studied: INV POR1374, Gulf of Tribugá, Morromico, 11.7 m, col. L. Chasqui, 08-20-2015. INV 
POR1377, Morromico, 11,9 m, col. L. Chasqui, 20-082015. INV POR1388, Punta Cruces, La Viuda, 13-15 m, col. L. Chasqui, 14-08-2015. INV POR1393 y 1394, Punta Cruces, La Mina, 15 m, col. L. Chasqui, 16-08-2015. INV POR1398 y 1399, cabo Marzo, Piedra de Eroito, 17,5 m, col. L. Chasqui, 15-08-2015.

Forma: masiva y lobular, de hasta $10-15 \mathrm{~cm}$ de diámetro y unos 5-10 cm de altura; superficie conulosa; ósculos en el tope de los lóbulos, usualmente con una membrana a manera de collar. Color: externamente amarillo oscuro (ocre) con tonos púrpura en vivo, interior amarillo; púrpura oscuro en alcohol. Consistencia: firme y ligeramente compresible. Ectosoma: pinacodermo fácilmente desprendible, sostenido por finales de las fibras del coanosoma, que conforman los cónulos. Coanosoma: red poligonal tridimensional de fibras de espongina con abertura de malla de 148-1037 $\mu \mathrm{m}$ de diámetro (Figura 23D). Fibras de 53-156 $\mu \mathrm{m}$ de diámetro, con corteza de color ámbar con protuberancias pequeñas y médula de 33-84 \% del diámetro (Figura 23C). Cerca de la superficie, las fibras se tornan dendríticas y generalmente se bifurcan y forman la superficie conular, que termina en puntas redondeadas, con un largo de 571-643 $\mu \mathrm{m}$.

Hábitat: abundante en los arrecifes rocosos del Pacífico norte colombiano.

Distribución: sur de Baja California a Oaxaca (México) (Gómez y Bakus, 1992; Cruz-Barraza et al., 2012; Vega, 2012); bahía Málaga en el Pacífico colombiano (Escobar, 2000); Pacífico norte colombiano.

Comentarios: se revisaron los estudios de Gómez y Bakus (1992) y Cruz-Barraza et al. (2012) del Pacífico mexicano. La presencia de una red poligonal tridimensional regular y las medidas del diámetro son similares en ambas descripciones; sin embargo, comparando la abertura de malla, es más pequeña que la que registran Gómez y Bakus (1992), 1,1-1,9 mm vs 0,2-1 $\mathrm{mm}$ en nuestro material. También se compararon las especies descritas por Gómez et al. (2018) del golfo de California. La forma de crecimiento y el color externo de nuestro material es similar a A. airapii, pero el esqueleto, en lugar de una malla uniplanar, cuenta con una red poligonal de donde surgen fibras dendríticas, lo cual también difiere de $A$. sinuscaliforniensis, que tiene un esqueleto totalmente reticulado, sin fibras dendríticas.
POR1377, Morromico, 11.9 m, col. L. Chasqui, 08-202015. INV POR1388, Punta Cruces, La Viuda, 13-15 m, col. L. Chasqui, 08-14-2015. INV POR1393 and 1394, Punta Cruces, La Mina, 15 m, col. L. Chasqui, 08-16-2015. INV POR1398 and 1399, Cape Marzo, Piedra de Eroito, 17.5 m, col. L. Chasqui, 08-15-2015.

Shape: massive and lobular, up to $10-15 \mathrm{~cm}$ in diameter and about $5-10 \mathrm{~cm}$ in height; conuloses surface; oscules at the top of the lobes, usually with a membrane as a collar. Color: externally dark yellow (ocher) with purple hues in vivid, yellow interior; dark purple in alcohol. Consistency: firm and slightly compressible. Ectosome: easily detachable pinacoderm, supported by the end of the fibers of the choanosome, which conform the conules. Choanosome: three-dimensional polygonal network of spongin fibers with a mesh size of 148-1037 $\mu \mathrm{m}$ in diameter (Figure 23D). Fibers 53-156 $\mu \mathrm{m}$ in diameter, with amber crust with small protrusions and pith 33-84 \% of the diameter (Figure 23C). Near the surface, the fibers become dendritic and generally bifurcate and form the conular surface, ending in rounded tips, 571-643 $\mu \mathrm{m}$ long.

Habitat: abundant in the rocky reefs of the Colombian North Pacific.

Distribution: southern Baja California to Oaxaca (Mexico) (Gómez and Bakus, 1992; Cruz-Barraza et al., 2012; Vega, 2012); Málaga Bay in the Colombian Pacific (Escobar, 2000); Colombian North Pacific.

Comments: the studies by Gómez and Bakus (1992) and Cruz-Barraza et al. (2012) from the Mexican Pacific were reviewed. The presence of a regular three-dimensional polygonal lattice and the diameter measurements are similar in both descriptions; however, comparing the mesh size, it is smaller than that recorded by Gómez and Bakus (1992), 1.1$1.9 \mathrm{~mm}$ vs $0.2-1 \mathrm{~mm}$ in our material. The species described by Gómez et al. (2018) from the Gulf of California was also compared. The growth form and the external color of our material is similar to A. airapii, but the skeleton, instead of a uniplanar mesh, has a polygonal network from which dendritic fibers arise, which also differs from $A$. sinuscaliforniensis, which has a fully cross-linked skeleton, without dendritic fibers. 

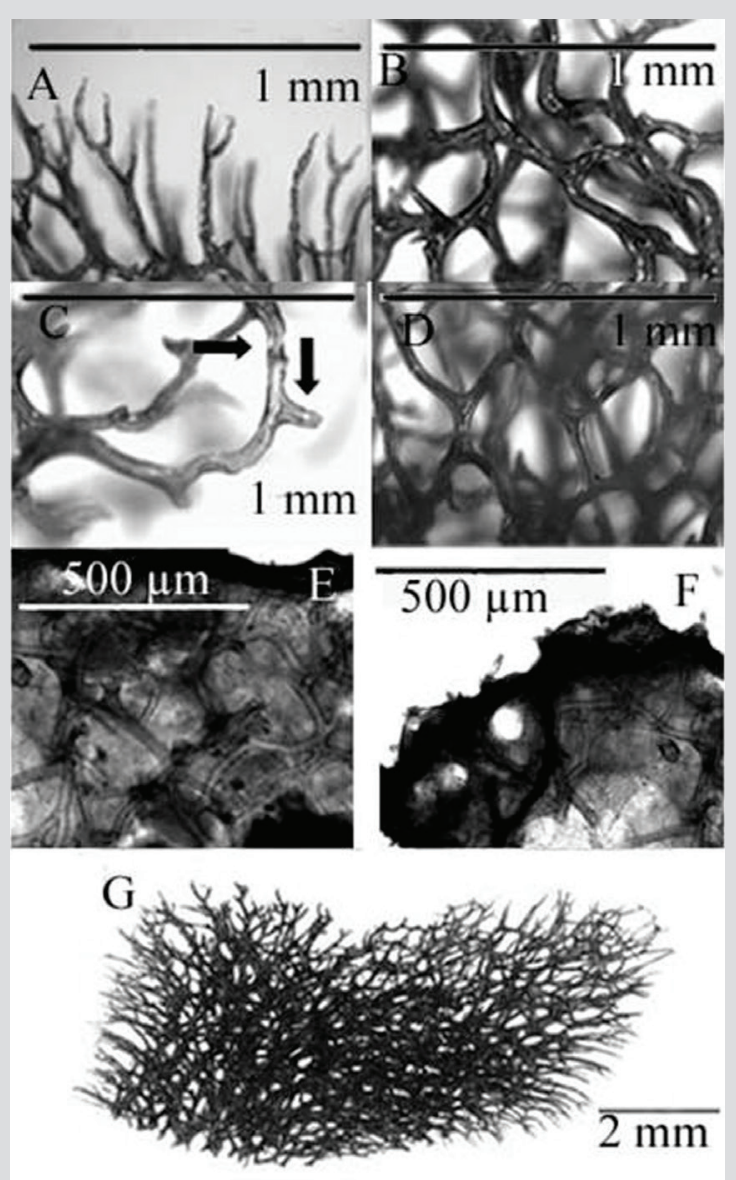

Figura 23. Aplysina gerardogreeni. A) Fibras dendríticas bifurcadas en la superficie. B) Médula de la fibra de espongina. C) Detalle de fibras con corteza y médula nodular y protuberancias cortas (señaladas con flechas). D) Red poligonal tridimensional. E) y F) Corte histológico. G) Retícula esquelética tridimensional (en $\mathbf{G}$ y en $\mathbf{A}$-D el tejido fue eliminado en baño de hipoclorito).

\section{Especie 23. Aplysina cf. revillagigedi Cruz-Barraza, Carballo, Rocha-Olivares, Ehrlich y Hog, 2012}

Figura 24; Lám. 3, fig. 4.

Aplysina revillagigedi Cruz-Barraza, Carballo, RochaOlivares, Ehrlich y Hog, 2012: 3, figs. 1B, C; 2F-H.

Material estudiado: INV POR1382, golfo de Tribugá, Morros de Jurubidá, 14,5 m, col. L. Chasqui, 20-08-2015. INV POR1390 y 1391, Punta Cruces, La Viuda, 13-15 m, col. L. Chasqui, 14-08-2015. INV POR1406, cabo Marzo, 18,5 m, col. L. Chasqui, 15-08-2015.

Forma: masiva amorfa, lobulada, de aproximadamente 5 $\mathrm{cm}$ de diámetro y hasta unos $3 \mathrm{~cm}$ de alto. Superficie lisa, cónulos evidentes en muestras preservadas, suaves al tacto. Ósculos dispersos o alineados en el tope de los lóbulos, 1,6-6,7 mm de diámetro. Color: amarillo brillante; púrpura
Figure 23. Aplysina gerardogreeni. A) Dendritic fibers bifurcated on the surface. B) Pith of the spongin fiber. C) Detail of fibers with nodular cortex and pith and short protrusions (indicated with arrows). D) Threedimensional polygonal network. E) and F) Histological section. G) Three-dimensional skeletal reticulation (in $\mathbf{G}$ and $\mathbf{A}-\mathbf{D}$ the tissue was removed in a bleach bath).
Figure 24; Plate 3, fig. 4.

Aplysina revillagigedi Cruz-Barraza, Carballo, RochaOlivares, Ehrlich and Hog, 2012: 3, figs. 1B, C; 2F-H.

Material studied: INV POR1382, Gulf of Tribugá, Morros de Jurubidá, $14.5 \mathrm{~m}$, col. L. Chasqui, 08-20-2015. INV POR1390 and 1391, Punta Cruces, La Viuda, 13-15 m, col. L. Chasqui, 08-14-2015. INV POR1406, Cape Marzo, 18.5 m, col. L. Chasqui, 08-15-2015.

Shape: massive amorphous, lobulated, about $5 \mathrm{~cm}$ in diameter and up to about $3 \mathrm{~cm}$ high. Smooth surface, conules evident in preserved specimens, soft to the touch. Scattered or aligned oscules at top of lobes, 1.6-6.7 mm diameter. Color: bright yellow; dark purple in alcohol. 
oscuro en alcohol. Consistencia: flexible, ligeramente compresible. Ectosoma: finales de la red coanosómica sostienen el pinacodermo. Coanosoma: esqueleto tridimensional reticulado, poligonal a irregular, y muy abierto, con mallas de 153-1037 $\mu \mathrm{m}$ de diámetro. Fibras de color ámbar o marrón oscuro, 119-60 $\mu \mathrm{m}$ de diámetro, con médula de 29-74 \% del diámetro (Figura 24A). Hacia la superficie, terminan a manera de fibras dendríticas de 326$1749 \mu \mathrm{m}$ de largo, con puntas redondeadas (Figura 24B).

Hábitat: arrecifes rocosos someros.

Distribución: Pacífico mexicano (Cruz-Barraza et al., 2012); Pacífico norte colombiano

Comentarios: la identificación es tentativa, pues es difícil separar las especies masivas de Aplysina del POT. La forma de crecimiento y la organización de la red de espongina se confunde con la de $A$. gerardogreeni, ya que algunas muestras son lóbulos tubulares con un ósculo apical, pero, en estas, la organización del esqueleto cerca de la superficie muestra fibras que se ramifican y terminan en puntas redondeadas como las de $A$. revillagigedi.
Consistency: flexible, slightly compressible. Ectosome: end of the choanosomal network supports the pinacoderm. Choanosome: three-dimensional cross-linked, polygonal to irregular, and very open skeleton, with meshes of 153-1037 $\mu \mathrm{m}$ in diameter. Amber or dark brown fibers, $119-60 \mu \mathrm{m}$ in diameter, with pith $29-74 \%$ of the diameter (Figure 24A). Towards the surface, they terminate as dendritic fibers 326$1749 \mu \mathrm{m}$ long, with rounded tips (Figure 24B).

Habitat: shallow rocky reefs.

Distribution: Mexican Pacific (Cruz-Barraza et al., 2012); Colombian North Pacific.

Comments: identification is tentative, as it is difficult to separate the massive Aplysina species from the ETP. The shape of growth and the organization of the spongin network is confused with that of $A$. gerardogreeni, since some samples are tubular lobes with an apical osculum, but, in these, the organization of the skeleton near the surface shows fibers that branch out and end in rounded tips like those of $A$. revillagigedi.
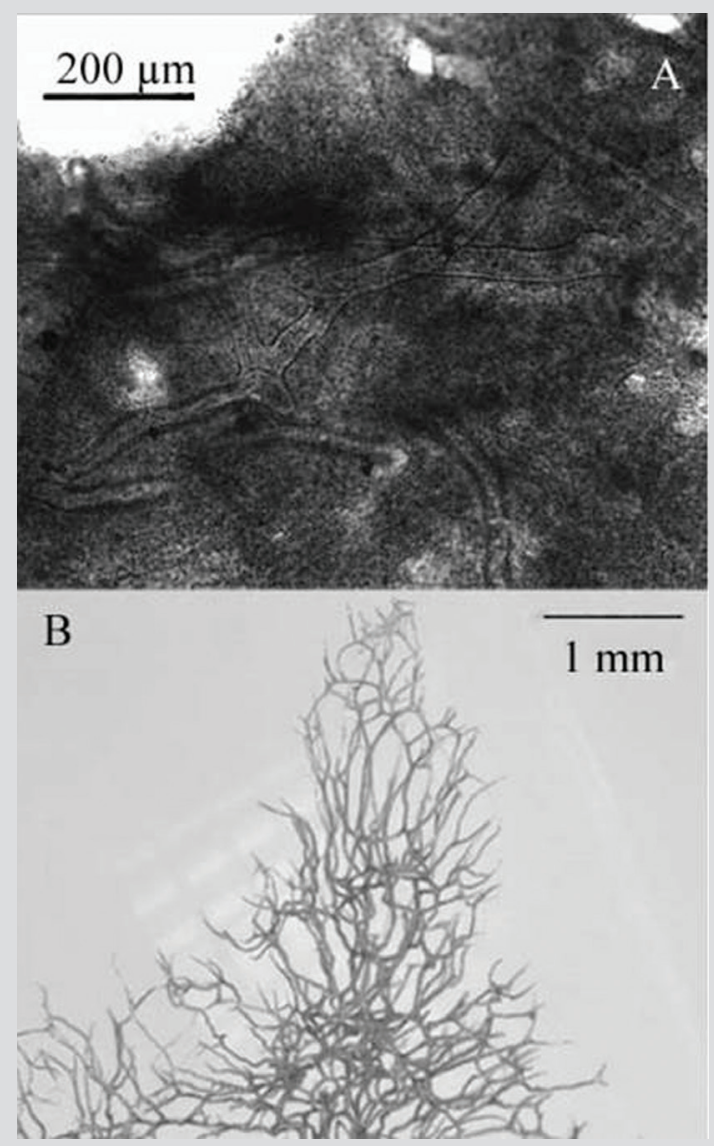

Figura 24. Aplysina cf. revillagigedi. A) Corte histológico. B) Reticulación esquelética tridimensional (tejido eliminado en baño de hipoclorito).
Figure 24. Aplysina cf. revillagigedi. A) Histological section. B) Threedimensional skeletal reticulation (tissue removed in a bleach bath). 
Familia Ianthellidae Hyatt, 1875

\section{Especie 24. Vansoestia sp.}

Figura 25; Lám. 3, fig. 5.

Material estudiado: INV POR1370, cabo Corrientes, Piedra de Colo, 14 m, col. L. Chasqui, 19-08-2015. INV POR1384, golfo de Tribugá, Morros de Jurubidá, 10 m, col. L. Chasqui, 20-08-2015.

Forma: revestimiento delgado extendiéndose irregular y fraccionadamente sobre el sustrato, por varios centímetros. Color: púrpura claro; púrpura oscuro en alcohol. Consistencia: suave. Ectosoma: pinacodermo con una especie de red de cordones de mayor densidad celular, con ojo de malla de 17-57 $\mu \mathrm{m}$ de diámetro (Figura 25C). Coanosoma: cordones granulosos de células, a manera de fibras, 96-182 $\mu \mathrm{m}$ de diámetro (Figura 25A, 25B). En los cortes y macerados se vislumbran fibras verticales cortas con gran acumulación de detritos (Figura 25D), pero son difíciles de discernir por lo pigmentado de las células.

Hábitat: sobre arrecife rocoso y otros organismos.

Distribución: Pacífico norte colombiano.

Comentarios: se incluyó en el género Vansoestia por su similitud con Vansoestia caribensis Díaz, Thacker, Redmond, Pérez y Collins, 2015 tanto en la morfología
Family Ianthellidae Hyatt, 1875

\section{Species 24. Vansoestia sp.}

Figure 25; Plate 3, fig. 5.

Material studied: INV POR1370, Cape Corrientes, Piedra de Colo, 14 m, col. L. Chasqui, 08-19-2015. INV POR1384, Gulf of Tribugá, Morros de Jurubidá, 10 m, col. L. Chasqui, 08-20-2015.

Shape: thin encrustation spreading irregularly and fractionally over the substratum, for several centimeters. Color: light purple; dark purple in alcohol. Consistency: soft. Ectosome: pinacoderm with a sort of net of strands of higher cell density, with a mesh size of 17-57 $\mu \mathrm{m}$ in diameter (Figure 25C). Choanosome: grainy fiber-like strands of cells, 96-182 $\mu \mathrm{m}$ in diameter (Figure 25A, 25B). Short vertical fibers with a large accumulation of debris are visible in sections and macerates (Figure 25D), but they are difficult to discern due to the pigmentation of the cells.

Habitat: on a rocky reef and other organisms.

Distribution: Colombian North Pacific.

Comments: this species was included in the genus Vansoestia due to its similarity with Vansoestia caribensis Díaz, Thacker, Redmond, Pérez, and Collins, 2015 both in external morphology and in the presence of granular strands.

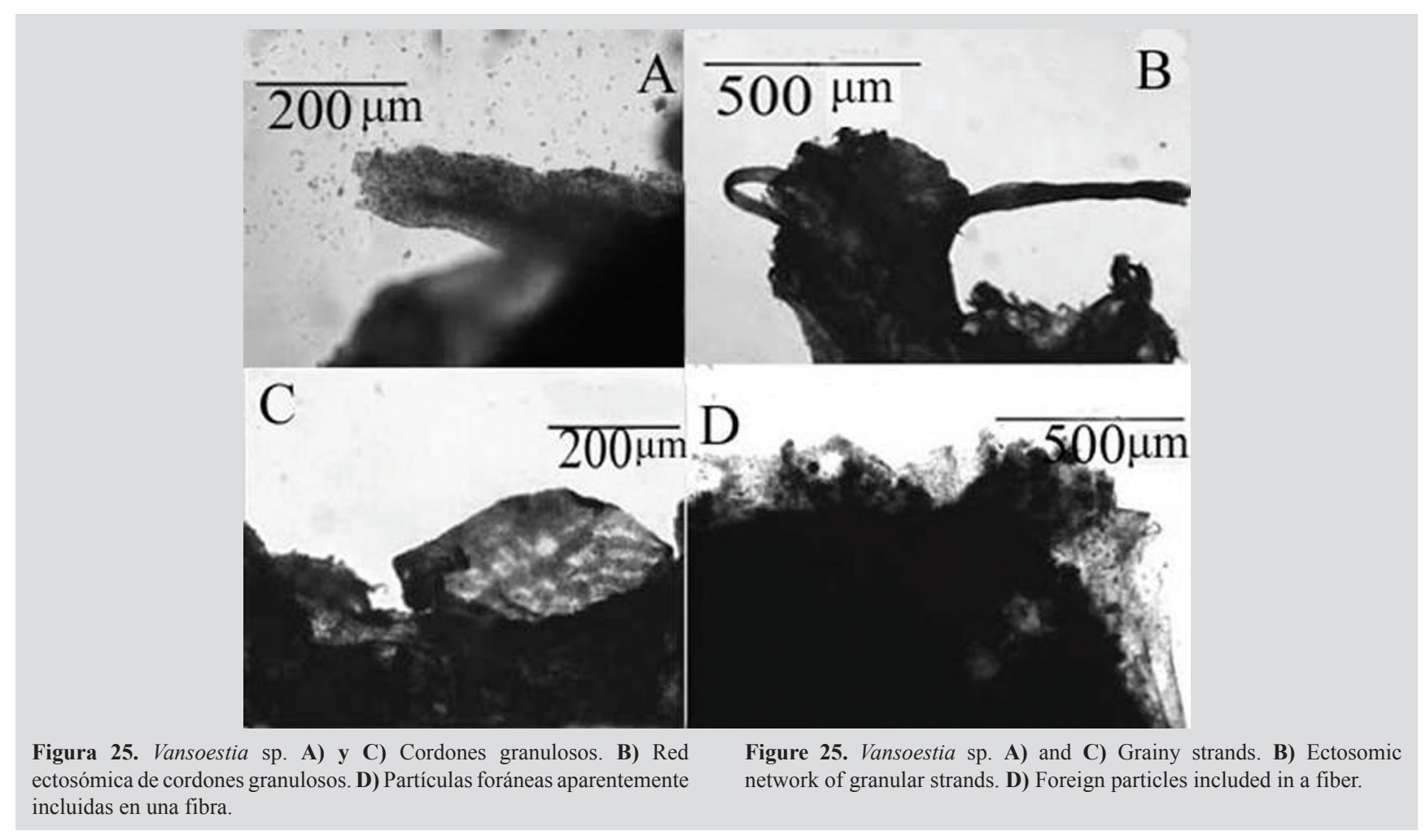


externa como en la presencia de cordones granulosos. No parece haber fibras de espongina tipo Aplysilla (limpias), pero podría haber fibras tipo Pleraplysilla (rellena de detritos); por ello, Zea et al. (2014) llamaron Pleraplysilla sp. a sus muestras de $V$. caribensis, por la presencia aparente de fibras verticales cortas no divididas, rellenas de detritos. Confirmarlo requiere de métodos histológicos diferentes como cortes desbastados y pulidos.

\section{DISCUSIÓN}

La diversidad conocida de esponjas en la región del POT, con no más de un centenar de especies (Van Soest et al., 2012), es mucho menor que la del Caribe, con al menos 519 especies (Miloslavich et al., 2010). La primera y más obvia razón es un esfuerzo de muestro desigual entre ambas regiones, lo cual fue notado por Van Soest et al. (2012). Mientras en el Caribe se considera que el grupo es bien conocido ( $>70 \%$ de las especies descritas, al menos cuatro guías de identificación; Miloslavich et al., 2010), la fauna de esponjas del POT está entre las menos conocidas globalmente, con solo cuatro estudios recientes que incluyen listas de especies (Escobar, 2000; Berman, 2004; Lizarazo, 2018; Carballo et al., 2019).

En Colombia, ese sesgo es aún más marcado, pues mientras para el Caribe existen más de 25 trabajos publicados sobre biodiversidad de esponjas (Zea, 1987; Zea et al., 2014), así como una guía de identificación (Zea, 1987), para el Pacífico colombiano se conocen solamente tres trabajos no publicados (Narváez, 1999; Escobar, 2000; Lizarazo, 2018). En consecuencia, mientras que para el Caribe colombiano se han registrado más de 150 especies de esponjas (Silva y Zea, 2017), para el Pacífico se conocen solo 46 especies. Sin embargo, esa diferencia en la biodiversidad de esponjas entre las dos costas va más allá de un mero sesgo de muestreo, como lo sugieren los resultados de Carballo et al. (2019). Ellos compararon la diversidad de esponjas en arrecifes coralinos del POT, el Caribe y el Pacífico Occidental, y muestran grandes diferencias en abundancia y riqueza de especies, así como en formas de crecimiento (masivas y expuestas en el Caribe y Pacífico Occidental versus pequeñas, incrustantes y crípticas en el POT). Observaciones realizadas durante el trabajo de campo en este estudio comparadas con observaciones de los autores en hábitats "similares" (i. e. arrecifes e islotes rocosos) de la costa Caribe sugieren que las observaciones de Carballo et al. (2019) sobre la marcada diferencia entre las comunidades de esponjas de arrecifes coralinos del
There do not appear to be Aplysilla-type fibers (clean), but there could be Pleraplysilla-type fibers (filled with debris); therefore, Zea et al. (2014) called Pleraplysilla sp. V. caribensis samples, due to the apparent presence of short, undivided vertical fibers, filled with detritus. Confirming this requires different histological methods such as ground and polished sections.

\section{DISCUSSION}

The known diversity of sponges in the ETP region, with no more than a hundred species (Van Soest et al., 2012), is much lower than that of the Caribbean, with at least 519 species (Miloslavich et al., 2010). The first and most obvious reason is an uneven sampling effort between both regions, which was noted by Van Soest et al. (2012). While in the Caribbean the group is considered to be well known ( $>70 \%$ of the described species, at least four identification guides; Miloslavich et al., 2010), the sponge fauna of the ETP is among the least known globally, with only four recent studies that include lists of species (Escobar, 2000; Berman, 2004; Lizarazo, 2018; Carballo et al., 2019).

In Colombia, this bias is even more marked, because while for the Caribbean there are more than 25 published works on sponge biodiversity (Zea, 1987; Zea et al., 2014), as well as an identification guide (Zea, 1987), for the Colombian Pacific, only three unpublished works are known (Narváez, 1999; Escobar, 2000; Lizarazo, 2018). Consequently, while for the Colombian Caribbean more than 150 sponge species have been recorded (Silva and Zea, 2017), for the Pacific only 46 species are known. However, this difference in sponge biodiversity between the two coasts goes beyond mere sampling bias, as suggested by the results of Carballo et al. (2019). They compared the diversity of sponges in coral reefs of the ETP, the Caribbean, and the Western Pacific, and showed large differences in abundance and richness of species, as well as in growth forms (massive and exposed in the Caribbean and Western Pacific versus small, encrusting and cryptic in the ETP). Observations made during fieldwork in this study compared with the authors' observations at "similar" habitats (i.e. reefs and rocky islets) off the Caribbean coast suggest that the observations of Carballo et al. (2019) on the marked difference between the coral reef sponge communities of the Mexican Pacific and the Caribbean also apply to the rocky reef ecosystems in Colombia. 
Pacífico mexicano y del Caribe aplican también para los ecosistemas de arrecifes rocosos en Colombia.

Varias explicaciones se han propuesto para esas diferencias en las comunidades de esponjas entre las dos regiones, que involucran factores biológicos ( $v . g$. depredación, competencia) y físicos (v.g. sedimentación, turbulencia, abrasión) a escala local, así como fenómenos a escala regional y global (v. g. surgencia, ENSO) (Wulff, 1997; Carballo et al., 2019). En cuanto a la diferencia en las formas de crecimiento predominantes en ambas costas (i.e. incrustantes y masivas pequeñas ocultas entre el arrecife en el POT versus grandes colonias erectas en la columna de agua en el Caribe), se cree que una fuerte presión de depredación en el POT, en especial de peces espongívoros, sobre especies que en su mayoría carecen de defensas químicas restringe la colonización y un mayor desarrollo en ambientes expuestos (Bakus, 1964; Wulff, 1997). Carballo et al. (2019) atribuyen esa fuerte diferencia en las comunidades de esponjas entre el Caribe y el POT al efecto de un acentuado régimen de perturbaciones naturales locales y de gran escala que actúan a largo plazo sobre una biota que quizá no fue nunca particularmente exuberante $[i$. e. hablando específicamente de esponjas asociadas a arrecifes coralinos, ver López-Pérez (2017) para un contexto general sobre origen de la biota coralina del POT] y que quedó virtualmente aislada hace alrededor de tres millones de años con el levantamiento del istmo de Panamá. Entonces, perturbaciones naturales de gran impacto como los cambios en temperatura superficial y régimen de nutrientes causados por el ENSO (Wang et al., 2017), así como eventos recurrentes de mareas bajas extremas, entre otros, pudieron generar procesos de extinción local y regional en comunidades de esponjas, lo que sumado a una ausencia de efecto de rescate debido al levantamiento del istmo y a la virtual imposibilidad de colonización natural de nuevas especies de esponjas a través de la barrera del Pacífico (i. e. más de $7000 \mathrm{~km}$ de aguas abiertas y profundas) podrían ser las fuerzas principales que han sentenciado una empobrecida fauna de Porifera en el POT.

Por otra parte, es bien conocida la marcada diferencia en biodiversidad marina entre el POT y el Caribe, especialmente en lo que se refiere a la biota de los arrecifes coralinos (Veron, 1995). En términos generales, una escasa presencia y desarrollo de formaciones coralinas en el POT afecta enormemente cualquier comparación de biodiversidad con el Caribe, la segunda región en desarrollo coralino del mundo, en cualquier taxón que esté fuertemente asociado a esos ecosistemas, como es el caso de las esponjas.
Several explanations have been proposed for these differences in sponge communities between the two regions, involving biological (e.g. predation, competition) and physical (e.g. sedimentation, turbulence, abrasion) factors on a local scale, as well as phenomena on a regional and global scale. (e.g. upwelling, ENSO) (Wulff, 1997; Carballo et al., 2019). Regarding the difference in the predominant growth forms on both coasts (i.e. encrusting and massive small hidden between the reef in the ETP versus large colonies growing upright into the water column in the Caribbean), it is believed that a strong predation pressure in ETP, especially of spongivorous fish, on species that mostly lack chemical defenses, restricts colonization and further development in exposed environments (Bakus, 1964; Wulff, 1997). Carballo et al. (2019) attribute this strong difference in sponge communities between the Caribbean and the ETP to the effect of an accentuated regime of local and largescale natural disturbances that act in the long term on a biota that was perhaps never particularly exuberant [i.e. speaking specifically of sponges associated with coral reefs, see López-Pérez (2017) for a general context on the origin of the coral biota of the ETP] and that was virtually isolated around three million years ago with the uprising of the Isthmus of Panama. Then, natural disturbances of great impact such as changes in surface temperature and nutrient regime caused by ENSO (Wang et al., 2017), as well as recurring events of extreme low tides, among others, could generate local and regional extinction processes in sponge communities, which added to an absence of rescue effect due to the uplift of the isthmus and the virtual impossibility of natural colonization of new sponge species through the Pacific barrier (i.e. more than $7000 \mathrm{~km}$ of open and deep water) could be the main forces that have sentenced an impoverished Porifera fauna in the ETP.

On the other hand, the marked difference in marine biodiversity between the ETP and the Caribbean is well known, especially with regard to the biota of coral reefs (Veron, 1995). In general terms, a scarce presence and development of coral formations in the ETP greatly affect any comparison of biodiversity with the Caribbean, the second region in coral development in the world, in any taxon that is strongly associated with these ecosystems, as in the case of sponges. Even, not only the low presence of coral reefs but of reefs in general (i.e. including rocky reefs) in the Colombian Pacific (e.g. Posada et al., 2009) affects the diversity indicators of sessile biota that requires hard substratum to settle. 
Incluso, no solo la baja presencia de arrecifes coralinos, sino de arrecifes en general (i.e. incluyendo arrecifes rocosos) en el Pacífico colombiano (v. g. Posada et al., 2009) afecta los indicadores de diversidad de la biota sésil que requiere sustrato duro para asentarse.

De las esponjas identificadas, seis especies son morfológicamente similares (especies geminadas) con especies del Caribe colombiano, lo que podría indicar que: 1. Se trata de especies hermanas con un ancestro común previo al cierre del istmo; 2 . Se trata de poblaciones de una misma especie quizá en un lento proceso de divergencia evolutiva vía especiación vicariante; 3 . Alguna de las dos poblaciones es una invasión biológica mediada por el transporte marítimo (i. e. como biofouling) vía canal de Panamá. La respuesta necesariamente pasa por el análisis genético molecular de un número de especímenes de esas "especies geminadas", el cual está fuera del alcance de este trabajo; sin embargo, este asunto deja ver que la historia evolutiva de poblaciones de especies marinas a ambos lados del istmo de Panamá sigue siendo de total relevancia y materia de interés.

En cuanto a la distribución geográfica de las esponjas encontradas en el Pacífico colombiano, se agruparon en cinco sectores biogeográficos (*especies registradas por Escobar (2000), **especies registradas por Narváez (1999): Pacífico Oriental Tropical y provincia costera californiana: Spheciospongia sp. (posiblemente $S$. raromisclerosa)*; Pacífico Oriental y provincia costera Chile-Perú: Scalarispongia similis; Pacífico Oriental Tropical (desde México hasta Ecuador): Axinella nayaritensis, Desmanthus levii, Crambe panamensis, Discorhabdella littoralis, Axinyssa isabela, Geodia media, Chondrosia tenochca, Aplysina chiriquiensis, A. gerardogreeni, Spirastrella sabogae*, Niphates perforata*, T. (Tedania) "nigrescens" (posiblemente T. (Tedania) tropicalis)*, A. azteca* (como Suberea), Aplysina cf. revillagigedi; Pacífico Oriental y Occidental Tropical: Chalinula nematifera, Chelonaplysilla violácea, Mycale cecilia*, Pseudosuberites "sulcatus"*; Tentativamente solo en Colombia mientras se define su estatus taxonómico (posiblemente nuevas especies, pero se requiere de estudios detallados): Prosuberites aff. laughlini, Dragmacidon sp., Acanthella sp., Placospongia aff. intermedia, Clathria (Microciona) sp., Scopalina aff. ruetzleri, Scopalina sp. 2., Epipolasis sp., Terpios sp., Aplysilla sp., Vansoestia sp., Xestospongia sp.**, Anphimedon sp.**, Aaptos sp.**, Geodia sp.**, Desmanthus aff. incrustans*, Geodia sp.*, Amorphinopsis sp.*, Topsentia aff. ophiraphidites*, Mycale sp.*, Haliclona sp. 1, Haliclona sp.
Of the sponges identified, six species are morphologically similar (geminated species) with species from the Colombian Caribbean, which could indicate that: 1. They are sister species with a common ancestor before the closure of the isthmus; 2 . These are populations of the same species, perhaps in a slow process of evolutionary divergence via vicariant speciation; 3 . Some of the two populations are a biological invasion mediated by maritime transport (i.e. as biofouling) via the Panama Canal. The answer necessarily goes through the molecular genetic analysis of several specimens of these "geminated species", which is outside the scope of this work; however, this issue shows that the evolutionary history of populations of marine species on both sides of the Isthmus of Panama continues to be of total relevance and matter of interest.

Regarding the geographical distribution of the sponges found in the Colombian Pacific, they were grouped into five biogeographic sectors (*Species recorded by Escobar (2000), **Species recorded by Narváez (1999): Eastern Tropical Pacific and Californian coastal province: Spheciospongia sp. (possibly S. raromisclerosa)*; Eastern Pacific and Chile-Peru coastal province: Scalarispongia similis; Tropical Eastern Pacific (from Mexico to Ecuador): Axinella nayaritensis, Desmanthus levii, Crambe panamensis, Discorhabdella littoralis, Axinyssa isabela, Geodia media, Chondrosia tenochca, Aplysina chiriquiensis, A. gerardogreeni, Spirastrella sabogae*, Niphates perforata*, T. (Tedania) "nigrescens" (possibly T. (Tedania) tropicalis)*, A. azteca* (as Suberea), Aplysina cf. revillagigedi; Tropical Eastern and Western Pacific: Chalinula nematifera, Chelonaplysilla violacea, Mycale cecilia*, Pseudosuberites "sulcatus"*; Tentatively only in Colombia while its taxonomic status is defined (possibly new species, but requires detailed studies): Prosuberites aff. laughlini, Dragmacidon sp., Acanthella sp., Placospongia aff. intermedia, Clathria (Microciona) sp., Scopalina aff. ruetzleri, Scopalina sp. 2., Epipolasis sp., Terpios sp., Aplysilla sp., Vansoestia sp., Xestospongia sp.**, Anphimedon sp.**, Aaptos sp.**, Geodia sp.**, Desmanthus aff. incrustans*, Geodia sp.*, Amorphinopsis sp. *, Topsentia aff. ophiraphidites*, Mycale sp. *, Haliclona sp. 1, Haliclona sp. 2, Haliclona sp. 3, Haliclona sp. 4*, Placospongia carinata ( $P$. intermedia in the Eastern Pacific), Aplysilla "sulphurea"*.

Finally, with the description made in this work of 24 species found in the rocky reefs of the Colombian North Pacific (21 are new records for Colombia), the number of 
2, Haliclona sp. 3, Haliclona sp. 4*, Placospongia carinata (P. intermedia en el Pacífico Oriental), Aplysilla "sulfurea"*.

Finalmente, con la descripción realizada en este trabajo de 24 especies halladas en los arrecifes rocosos (riscales y morros) del Pacífico norte colombiano (21 son nuevos registros para Colombia), se eleva a 46 el número de especies de esponjas para el Pacífico colombiano. Esta riqueza de especies se considera baja en comparación con la biodiversidad conocida del Caribe colombiano $(>150$ especies). Algunas de las esponjas aquí registradas tienen características morfológicas externas y del esqueleto muy similares a especies del Caribe, lo que indica que podrían ser especies geminadas; sin embargo, el análisis realizado con base en caracteres exclusivamente morfológicos no permite concluir si son o no especies diferentes y si requieren de un nuevo nombre; por tanto, la pregunta queda abierta a la espera de análisis más detallados, que incluyan la revisión de un mayor número de muestras de esas especies proveniente de ambas costas, y desde luego, del análisis de caracteres moleculares.

\section{AGRADECIMIENTOS}

El trabajo fue financiado por el Instituto de Investigaciones Marinas y Costeras (Invemar), a través del proyecto "Investigación científica hacia la generación de información y conocimiento de las zonas marinas y costeras de interés de la nación" (código BPIN 2017011000113) y hace parte del estudio "Caracterización biótica de los arrecifes rocosos con desarrollo significativo en el Pacífico norte colombiano". Agradecemos a José Luis Carballo por la confirmación de la especie Axinella nayaritensis; a Mateo López V. y a otro revisor anónimo cuyos comentarios contribuyeron a mejorar el manuscrito; a Eroito Álvarez, Luisa Ruiz, Arnold Llorente, Rodrigo Fajardo, y a Turquí Paradise Hotel and Dive Center por brindar facilidades logísticas y apoyo durante el trabajo de campo. Gracias a Felipe Valencia por elaborar el mapa del área de estudio y a Felipe Gentil por apoyar en la edición de imágenes. La colecta y depósito de muestras siguen las indicaciones del Decreto 309-2003 del Ministerio de Ambiente y Desarrollo Sostenible. Contribución número 1276 del Invemar. El trabajo de S. Zea es la contribución 500 del Instituto de Estudios en Ciencias del Mar (Cecimar), Universidad Nacional de Colombia, sede Caribe. sponge species for the Colombian Pacific rises to 46. This richness of species is considered low compared to the known biodiversity of the Colombian Caribbean ( $>150$ species). Some of the sponges recorded here have skeletal and external morphological characteristics very similar to Caribbean Species, which indicates that they could be geminated species; however, the analysis carried out based on exclusively morphological characters does not allow to conclude whether or not they are different species and whether they require a new name; therefore, the question remains open pending more detailed analyzes, which include the review of a greater number of samples of these species from both coasts, and of course, the analysis of molecular characteristics.

\section{ACKNOWLEDGEMENT}

The work was funded by the Institute for Marine and Coastal Research (Invemar), through the project "Scientific research towards the generation of information and knowledge of the marine and coastal areas of interest to the nation" (code BPIN 2017011000113) and is part of the study "Biotic characterization of rocky reefs with significant development in the Colombian North Pacific". We thank José Luis Carballo for the confirmation of the species Axinella nayaritensis; to Mateo López V. and another anonymous reviewer whose comments contributed to improving the manuscript; Eroito Álvarez, Luisa Ruiz, Arnold Llorente, Rodrigo Fajardo, and Turquí Paradise Hotel and Dive Center for providing logistical facilities and support during fieldwork. Thanks to Felipe Valencia for preparing the map of the study area and to Felipe Gentil for helping with the image editing. The collection and deposit of samples follow the indications of Decree 309-2003 of the Ministry of Environment and Sustainable Development. Contribution number 1276 from Invemar. The work of S. Zea is the contribution 500 of the Institute of Studies in Marine Sciences (Cecimar), National University of Colombia, Caribbean Campus. 

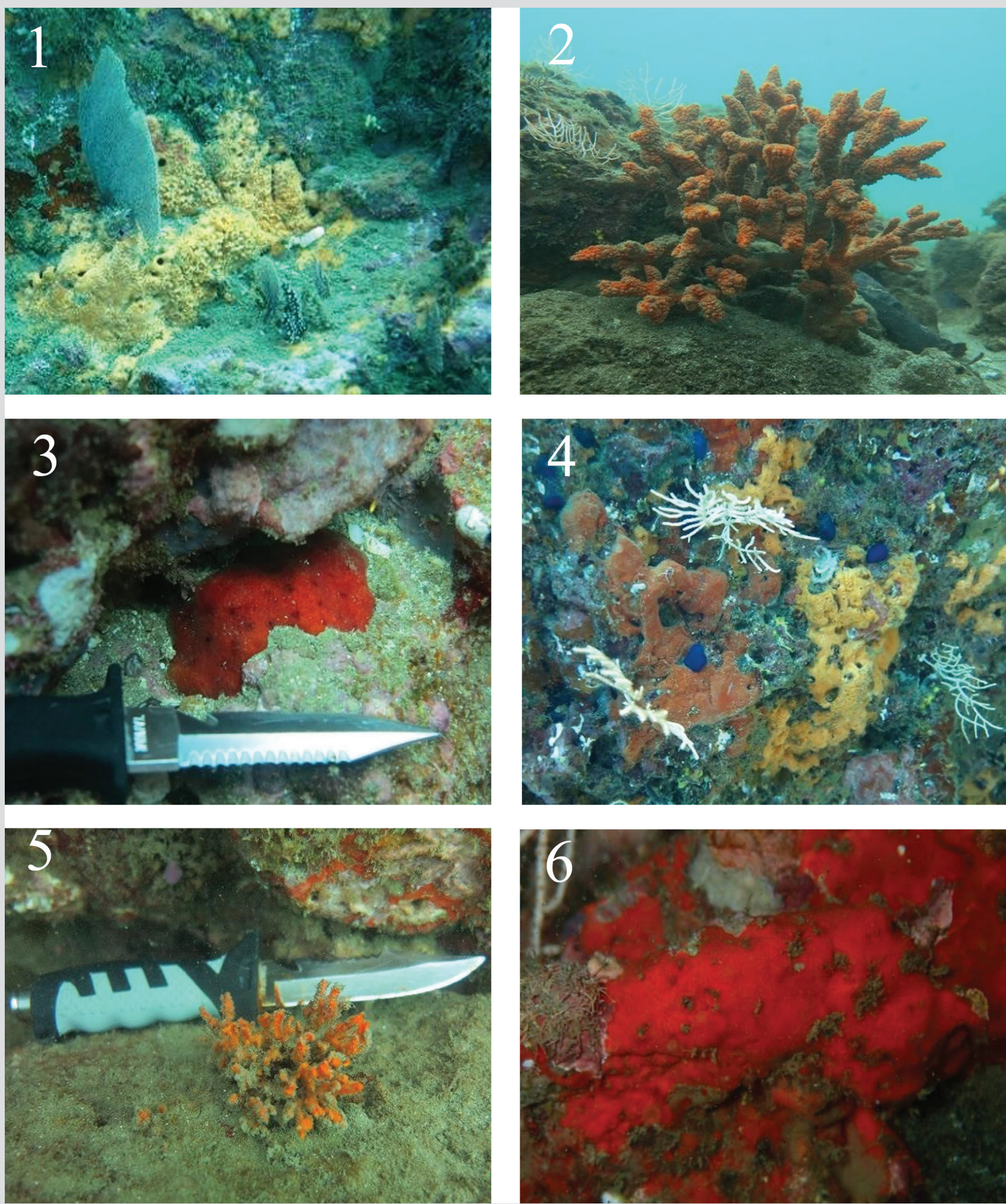

LÁMINA 1. fig. 1. Prosuberites aff. laughlini INV POR1361 con octocoral, La Parguera-Piñas, $13 \mathrm{~m}$; fig. 2. Axinella nayaritensis INV POR1372, golfo de Tribugá, $7.8 \mathrm{~m}$; fig. 3. Dragmacidon sp. INV POR1362, La Parguera-Piñas, $6 \mathrm{~m}$; fig. 4. Desmanthus levii (naranja rojizo, izq.), INV POR1376, golfo de Tribugá, $12 \mathrm{~m}$, con Prosuberites aff. laughlini (naranja pálido, der.); fig. 5. Acanthella sp., INV POR1373, golfo de Tribugá, $7.1 \mathrm{~m}$; fig. 6. Crambe panamensis., INV POR1365, La Parguera, $6 \mathrm{~m}$.

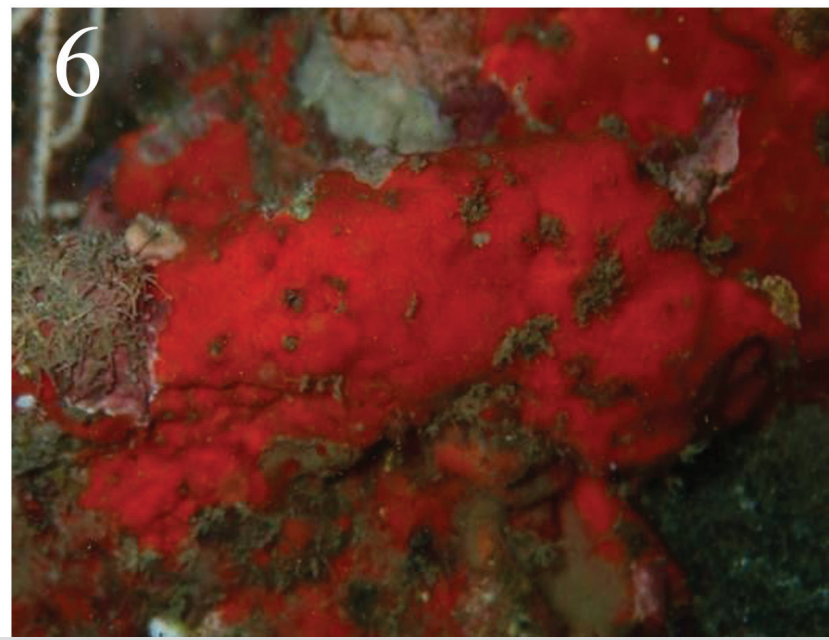

PLATE 1. fig. 1. Prosuberites aff. laughlini INV POR1361 with octocoral, La Parguera-Piñas, $13 \mathrm{~m}$; fig. 2. Axinella nayaritensis INV POR1372, Gulf of Tribugá, $7.8 \mathrm{~m}$; fig. 3. Dragmacidon sp. INV POR1362, La Parguera-Piñas, $6 \mathrm{~m}$; fig. 4. Desmanthus levii (reddishorange, left), INV POR1376, Gulf of Tribugá, $12 \mathrm{~m}$, with Prosuberites aff. laughlini (pale orange, right); fig. 5. Acanthella sp., INV POR1373, Gulf of Tribugá, $7.1 \mathrm{~m}$; fig. 6. Crambe panamensis., INV POR1365, La Parguera, $6 \mathrm{~m}$ 

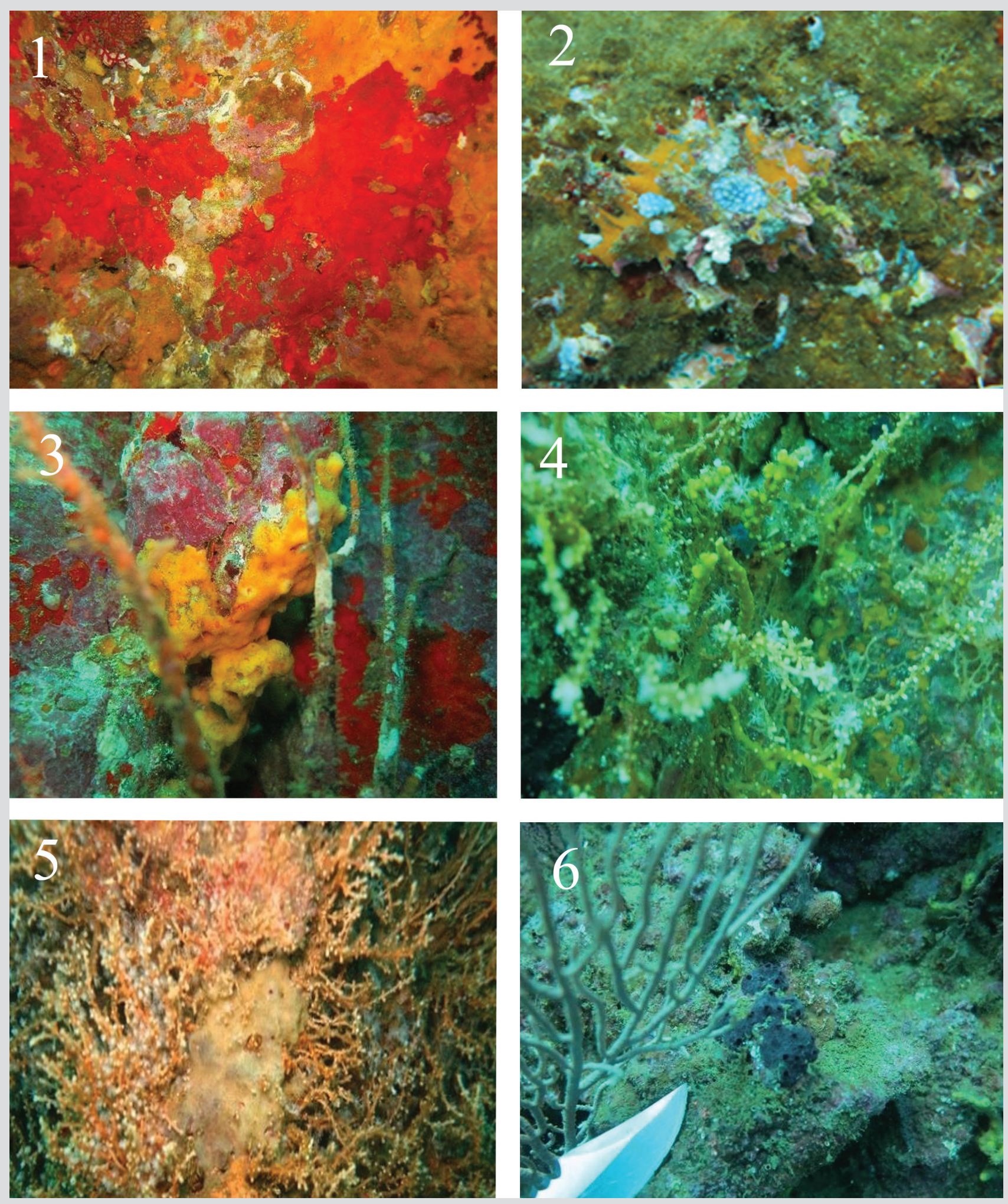

LÁMINA 2. fig. 1. Clathria (Microciona) sp., INV POR1400, cabo Marzo, 17 m; fig. 2. Scopalina sp. 2., INV POR1396, Punta Cruces, 15 $\mathrm{m}$ (revestimiento naranja sobre caracol); fig. 3. Axinyssa isabela, INV POR1397, cabo Marzo, 19 m; fig. 4. Terpios sp., INV POR1403, cabo Marzo, $17 \mathrm{~m}$ (revestimiento amarillo sobre octocoral Carijoa riisei); fig. 5. Aplysilla sp., INV POR1366, cabo Corrientes, $18 \mathrm{~m}$ (con octocoral Carijoa riisei); fig. 6. Chelonaplysilla violacea sensu Gómez et al., 2002, INV POR1368, cabo Corrientes, $19 \mathrm{~m}$.

PLATE 2. fig. 1. Clathria (Microciona) sp., INV POR1400, Cape Marzo, 17 m; fig. 2. Scopalina sp. 2., INV POR1396, Punta Cruces, 15 m (orange cladding on snail); fig. 3. Axinyssa isabela, INV POR1397, Cape Marzo, 19 m; fig. 4. Terpios sp., INV POR1403, Cape Marzo, 17 m (yellow encrustation on octocoral Carijoa riisei); fig. 5. Aplysilla sp., INV POR1366, Cape Corrientes, $18 \mathrm{~m}$ (with octocoral Carijoa riisei); fig. 6. Chelonaplysilla violacea sensu Gómez et al., 2002, INV POR1368, Cape Corrientes, $19 \mathrm{~m}$. 

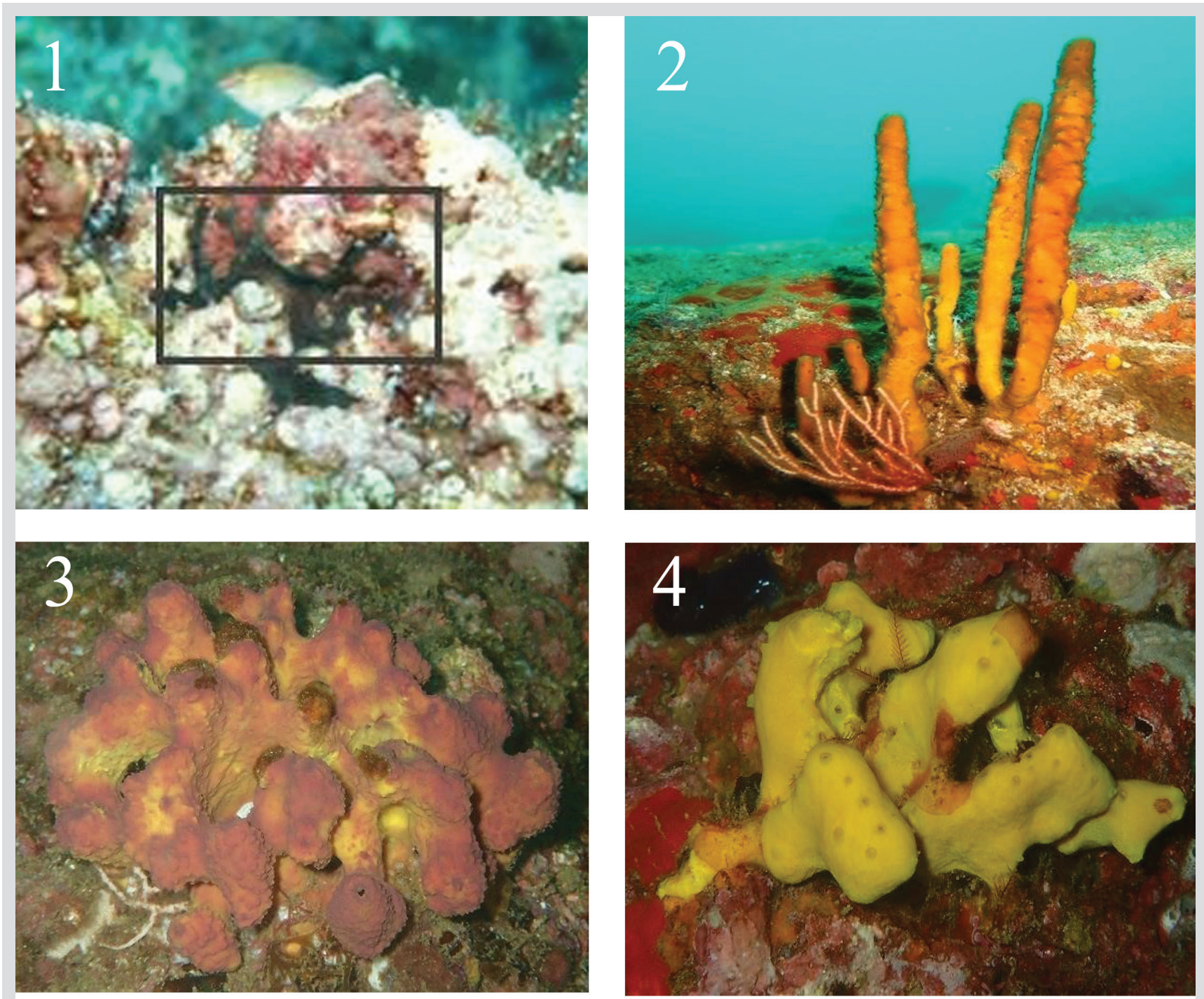

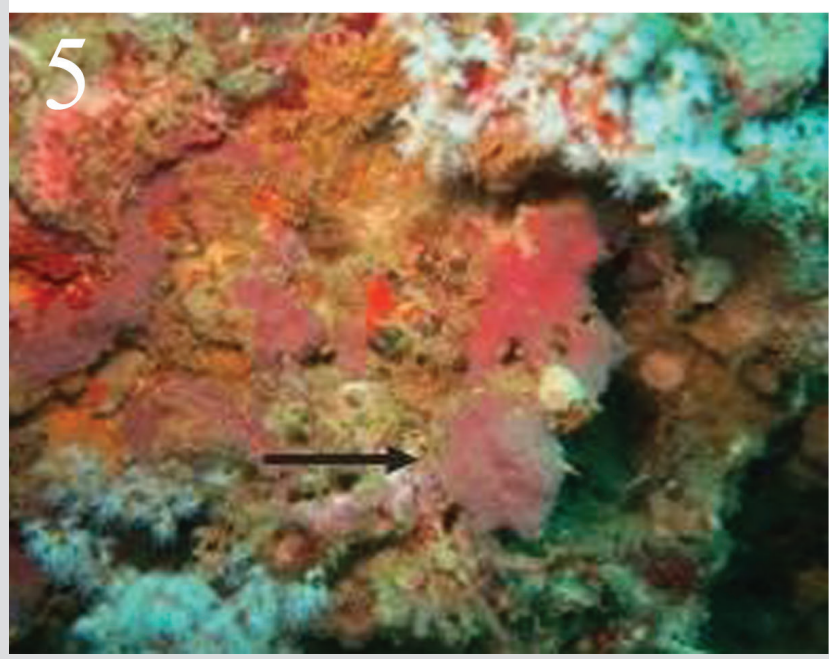

LÁMINA 3. fig. 1. Chondrosia tenochca, INV POR1371, cabo Corrientes, $11 \mathrm{~m}$. (en cuadro); fig. 2. Aplysina chiriquiensis, INV POR1402, cabo Marzo, $18.3 \mathrm{~m}$; fig. 3. Aplysina gerardogreeni, INV POR1388, Punta Cruces, 13-15 m; fig. 4. Aplysina cf. revillagigedi, INV POR1390, Punta Cruces, 13-15 m; fig. 5. Vansoestia sp., INV POR1370, cabo Corrientes, $14 \mathrm{~m}$ (señalada con flecha).
PLATE 3. fig. 1. Chondrosia tenochca, INV POR1371, Cape Corrientes, $11 \mathrm{~m}$. (in box); fig. 2. Aplysina chiriquiensis, INV POR1402, Cape Marzo, $18.3 \mathrm{~m}$; fig. 3. Aplysina gerardogreeni, INV POR1388, Punta Cruces, 13-15 m; fig. 4. Aplysina cf. revillagigedi, INV POR1390, Punta Cruces, 13-15 m; fig. 5. Vansoestia sp., INV POR1370, Cape Corrientes, $14 \mathrm{~m}$ (indicated with arrow). 


\section{BIBLIOGRAFÍA/LITERATURE CITED}

Aguirre, L.K., Y. Hooker, W. Philippe, and E. Hajdu. 2011. A new Clathria (Demospongiae, Microcionidae) from Peru occurring on rocky substrates as well as epibiontic on Eucidaris thouarsii sea urchins. Zootaxa, 3085: 41-54. https://doi.org/10.11646/zootaxa.3085.1.3

Alcolado, P.M. 1984. Nuevas especies de esponjas encontradas en Cuba (new species of sponges from Cuba). Poeyana, 271: 1-22.

Álvarez, B. and J.N.A. Hooper. 2002. Family Axinellidae Carter, 1875. 724-747. In: Hooper, J.N.A., R.W.M. Van Soest, and P. Willenz. (Eds.). Systema Porifera. A guide to the classification of sponges. Springer: Boston. 1100 p. https://doi.org/10.1007/978-1-4615-0747-5_80

Austin, W.C., B.S. Ott, H.M. Reiswig, P. Romagosa, and N.G. McDaniel. 2013. Two new species in the family Axinellidae (Porifera, Demospongiae) from British Columbia and adjacent waters. ZooKeys, 338: 11-28. https://doi.org/10.3897/zookeys.803.22543

Ávila, E. and J.L. Carballo. 2009. A preliminary assessment of the invasiveness of the Indo-Pacific sponge Chalinula nematifera on coral communities from the tropical Eastern Pacific. Biol. Inv., 11: 257-264. https://doi.org/10.1007/s10530-008-9230-5

Bakus, G.J. 1964. The effects of fish-grazing on invertebrate evolution in shallow tropical waters. Allan Hancock Fund. Publ. Occ., 27:1-29.

Bergquist, P.R. 1978. Sponges. Hutchinson, London. 268 p.

Berman, J. 2004. Sponge diversity in coral frameworks and coral communities within the Las Perlas archipelago, Panama. M. Sci. Thesis, Heriot-Watt University, Edinburgh. 83 p.

Boury-Esnault, N. and M.T. Lopes. 1985. Les Démosponges littorales de l’Archipel des Açores. Ann. Inst. Oceanogr. París, 61(2): $149-225$.

Boury-Esnault, N., M. Klautau, C. Bézac, J. Wulff, and A.M. Solé-Cava. 1999. Comparative study of putative conspecific sponge populations from both sides of the Isthmus of Panama. J. Mar. Biol. Ass. U.K., 79: 39-50. https://doi.org/10.1017/S0025315498000046

Bowerbank, J.S. 1858. On the anatomy and physiology of the Spongiadae. Part I. On the Spicula. Philos. Trans. Royal Soc. A., 148(2): $279-332$.

Bowerbank, J.S. 1862. On the anatomy and physiology of the Spongiadae. Part II. Philos. Trans. Royal Soc., 152(2): 747-829.

Bowerbank, J.S. 1873. Contributions to a general history of the Spongiadae. Proc. Zool. Soc. Lond., 3: 3-25.

Burton, M. 1940. Las esponjas marinas del Museo Argentino de Ciencias Naturales. Parte 1. Anales del Museo Argentino de Ciencias Naturales Bernardino Rivadavia, 40(6): 95-121.

Carballo, J.L. and J.A. Cruz-Barraza. 2008. First record of Axinyssa Lendenfeld, 1897 (Demospongiae, Halichondrida) from the East Pacific Ocean, with the description of Axinyssa isabela sp. nov. Zootaxa, 1784: 58-68. https://doi.org/10.11646/zootaxa.1784.1.4

Carballo, J.L., P. Gómez, J.A. Cruz-Barraza, and D.M. Flores-Sánchez. 2003. Sponges of the family Chondrillidae (Porifera: Demospongiae) from the Pacific coast of Mexico, with the description of three new species. Proc. Biol. Soc. Wash., 116(2): 515-527.

Carballo, J.L., J.A. Cruz-Barraza, and P. Gómez. 2004. Taxonomy and description of clionaid sponges (Hadromerida, Clionaidae) from the Pacific Ocean of México. Zool. J. Linn. Soc., 141(3): 353-397. https://doi.org/10.1111/j.1096-3642.2004.00126.x

Carballo, J.L., E. Bautista-Guerrero and J.A. Cruz-Barraza. 2018. Description and molecular phylogeny of Axinella nayaritensis n. sp. (Porifera: Axinellida) from East Pacific and remarks about the polyphyly of the genus Axinella. Zootaxa, 4482(1): 111-124. https://doi.org/10.11646/zootaxa.4482.1.4

Carballo, J.L., J.A. Cruz-Barraza, C. Vega, H. Nava, and M.C. Chávez-Fuentes. 2019. Sponge diversity in Eastern Tropical Pacific coral reefs: an interoceanic comparison. Sci. Rep., 9: 9409. https://doi.org/10.1038/s41598-019-45834-4

Cárdenas, P., T. Pérez, and N. Esnault. 2012. Sponge systematics facing new challenges. 79-209. In: Becerro, M.A., M.J. Uriz, M. Maldonado, and X. Turon. (Eds.). Advances in sponge science: phylogeny, systematics, ecology. Elsevier, 61. https://doi.org/10.1016/B978-0-12-387787-1.00010-6

Carter, H.J. 1875. Notes introductory to the study and classification of the Spongida. Part II. Proposed classification of the Spongida. Ann. Mag. nat. Hist., 4(16): 126-145.

Carter, H.J. 1883. Contributions to our knowledge of the Spongida. Ann. Mag. Nat. Hist., 11(65): 344-369. https://doi.org/10.1080/00222938309459163

Chombard, C. and N. Boury-Esnault. 1999. Good congruence between morphology and molecular phylogeny of Hadromerida, or how to bother sponge taxonomists. Mem. Qld. Mus., 44: 100.

Cook, S. and P.R. Bergquist. 2000. Two new genera and five new species of the Cacospongia group (Porífera, Demospongiae, Dictyoceratida). Zoosystema, 22(2): $383-400$.

Costa, G., G. Bavastrello, M. Pansini, and M. Bertolino. 2020. Acanthella danerii sp. nov. (Demospongiae, Bubarida, Dictyonellidae) from Chilean fjords (South Pacific Ocean). Zootaxa, 4790(2): 393-396. https://doi.org/10.11646/zootaxa.4790.2.13

Cruz-Barraza, J.A. and J.L. Carballo. 2008. Taxonomy of sponges (Porifera) associated with corals from the Mexican Pacific Ocean. Zool. Stud., 47(6): 741-758. 
Cruz-Barraza, J.A., J.L. Carballo, A. Rocha-Olivares, H. Ehrlich, and M. Hog. 2012. Integrative taxonomy and molecular phylogeny of genus Aplysina (Demospongiae: Verongida) from Mexican Pacific. PLoS ONE, 7(8): 42-49. https://doi.org/10.1371/journal.pone.0042049

De Laubenfels, M.W.1930. The sponges of California. Abstracts of dissertations for the degree of Doctor of Philosophy. Stanford Univ. Bull., 5(98): 24-29.

De Laubenfels, M.W. 1932. The marine and fresh water sponges of California. Proc. U.S. Nat. Mus., 81(2927): 1-140. https://doi.org/10.5479/si.00963801.812927.1

De Laubenfels, M.W. 1935. Some sponges of lower California (México). Am. Mus. Nov., 779: 1-14.

De Laubenfels, M.W. 1936a. A discussion of the sponge fauna of the Dry Tortugas in particular and the West Indies in general, with material for a revision of the families and orders of the Porifera. Carnegie Inst. Wash. Publ., 467: 1-225.

De Laubenfels, M.W. 1936b. A comparison of the shallow-water sponges near the Pacific end of the Panamá canal with those at the Caribbean end. Proc. U.S. Natl. Mus., 83: 441-454.

De Laubenfels, M.W. 1954. The sponges of the West-Central Pacific. Zool. Stud., 7. Oregon State College, Corvallis. 306 p. https://doi.org/10.5962/bhl. title.6516

De Weerdt, W.H. 2002. Family Chalinidae Gray, 1867. 852-873. In: Hooper, J.N.A., R.W.M. Van Soest, and P. Willenz (Eds.). Systema Porifera. A guide to the classification of sponges. Springer: Boston. 1100 p. https://doi.org/10.1007/978-1-4615-0747-5_91

Desqueyroux-Faúndez, R. and R.W.M. Van Soest. 1997. Shallow waters Demosponges of the Galápagos Islands. Rev. Suisse Zool., 104(2): $379-467$.

Díaz, J.M., L. Guillot y M.C. Velandia. 2016. La pesca artesanal en la costa norte del Pacífico colombiano: un horizonte ambivalente. Fundación Marviva, Bogotá. 158 p.

Díaz, M.C., B. Álvarez, and R.W.M. Van Soest. 1987. New species of Demospongiae (Porifera) from the national park Archipiélago de Los Roques, Venezuela Bijdr. Dierkd, 57(1): 31-41. https://doi.org/10.1163/26660644-05701003

Díaz, M.C., S.A. Pomponi, and R.W.M. Van Soest. 1993. A systematic revision of the central West Atlantic Halichondrida (Demospongiae, Porifera). Part III: Description of valid species. Sci. Mar., 57(4): 283-306.

Díaz, M.C., R.W.M. Van Soest, K. Rützler, and H.M Guzmán. 2005. Aplysina chiriquiensis, a new pedunculate sponge from the Gulf of Chiriquí, Panamá, Eastern Pacific (Aplysinidae, Verongida). Zootaxa, 1012: 1-12. https://doi.org/10.11646/zootaxa.1012.1.1

Díaz, M.C., R.W. Thacker, N.E. Redmond, T. Pérez, and A.G. Collins. 2015. Vansoestia caribensis gen. nov., sp. nov.: first report of the family Ianthellidae (Verongida, Demospongiae) in the Caribbean. Zootaxa, 3956(3): 403-412. https://doi.org/10.11646/zootaxa.3956.3.5

Erpenbeck, D., P. Sutcliffe, S. Cook, A. Dietzel, M. Maldonado, R.W.M. Van Soest, J.N.A. Hooper, and G. Wörheide. 2012. Horny sponges and their affairs: On the phylogenetic relationships of keratose sponges. Elsevier, 63(3): 809-816. https://doi.org/10.1016/j.ympev.2012.02.024

Escobar, T. 2000. Inventario y estudio taxonómico de las esponjas (Phylum Porifera) de algunas áreas del Pacífico colombiano. Tesis Biol. Mar. Univ. Valle, Buenaventura. $149 \mathrm{p}$.

García-Suárez, S.D., A. Acosta, E. Londoño-Cruz y J.R. Cantera K. 2012. Organismos sésiles y móviles del litoral rocoso: en el Pacífico colombiano: una guía visual para su identificación. Ser. Doc. Esp., (26). Invemar, Santa Marta. 133 p.

Goeij, J.M., H. van den Berg, M.M. van Oostveen, E.H. Epping, and F.C. Van Duyl. 2008. Major bulk dissolved organic carbon (DOC) removal by encrusting coral reef cavity sponges. Mar. Ecol. Prog. Ser., 357: 139-151. https://doi.org/10.3354/meps07403

Goeij, J.M., D. Oevelen, M.J. Vermeij, R. Osinga, J.J. Middelburg, A.F. de Goeij, and W. Admiraal. 2013. Surviving in a marine desert: the sponge loop retains resources within coral reefs. Science, 342: 108-110. https://doi.org/10.1126/science.1241981

Gómez, P. and G. Bakus. 1992. Aplysina gerardogreeni and Aplysina aztecus (Porifera: Demospongiae) new species from the Mexican Pacific. An. Inst. Cienc. Mar Limnol., 19(2): 175-180.

Gómez, P., J.L. Carballo, L.E. Vázquez, and J.A. Cruz. 2002. New records for the sponge fauna (Porifera: Demospongiae) of the Pacific coast of México (eastern Pacific Ocean). Proc. Biol. Soc. Wash., 115(1): 223-237.

Gómez, P., B. González-Acosta, C. Sánchez-Ortíz, Z. Hoffman, and C. Hernandéz-Guerrero. 2018. Amended definitios for Aplysinidae and Aplysina (Porifera, Demospongiae, Verongiida): on three new species from a remarkable population in the Gulf of California. Zootaxa, 4455(2): 322-342. https:// doi.org/10.11646/zootaxa.4455.2.4

Grant, R.E. 1861. Tabular view of the primary divisions of the Animal Kingdom, intended to serve as an outline of an elementary course of recent zoology (caino-zoology); or, the natural history of existing animals. Walton and Maberly, London. 91 p.

Gray, J.E. 1867. Notes on the arrangement of sponges, with the descriptions of some new genera. Proc. Zool. Soc. Lond., (2): $492-558$. 
Green, G. y P. Gómez. 1986. Estudio taxonómico de las esponjas de Mazatlán Sinaloa, México. An. Inst. Cienc. Mar Limnol. Univ. Nal. Autón. México, 13(3): 273-300.

Guzmán, H., C.A. Guevara, and O. Breedy. 2004. Distribution, diversity, and conservation of coral reefs and coral communities in the largest marine protected area of Pacific Panamá (Coiba Island). Environ. Conserv., 31: 111-121. https://doi.org/10.1017/S0376892904001250

Hartman, W.D. 1980. Systematics of the Porifera. 24-51. En: Hartman, W.D., J.W. Wendt y F. Wiedenmayer. (Eds.). Living and fossil sponges, notes for a short course. Univ. Miami, Miami. 274 p.

Holmes, B. and H. Blanch. 2007. Genus-specific associations of marine sponges with group I crenarchaeotes. Mar. Bio., 150: 759-772. https://doi. org/10.1007/s00227-006-0361-x

Hooper, J.N.A., R.W.M. Van Soest, and P. Willenz. (Eds.). 2002. Systema Porifera. A guide to the classification of sponges. Springer: Boston. 1100 p. https:// doi.org/10.1007/978-1-4615-0747-5

Hyatt, A. 1875. Revision of the North American Poriferae; with remarks upon foreign species. Part I. Mem. Read. Boston Soc. Nat. Hist., 2: $399-408$.

Lendenfeld, R. von. 1883. Über Coelenteraten der Südsee. II.Mittheilung. Neue Aplysinidae. Z. wiss. Zool., 38(2): 234-313.

Lendenfeld, R. von. 1910. The Sponges. 1. Geodiidae. En: Reports of the expeditions to the Eastern Tropical Pacific, in the charge of Alexander Agassiz, by the U.S. Fish Commission Steamer Albatross. Mem. Mus. Comp. Zool. Harv. Coll., 41(1): 224-259.

Lévi, C. 1953. Sur une nouvelle classification des Démosponges. C. r. hebd. séances Acad. sci, 236(8): 853-855.

Lévi, C. 1963. Spongiaires d'Afrique du Sud. Poecilosclérides. Trans. R. Soc. S. Afr., 37(1): 1-72.

Lizarazo, N. 2018. Esponjas asociadas al ecosistema rocoso (Riscales y Morros) del Pacífico norte chocoano, Colombia. Tesis Biol. Mar. Univ. Jorge Tadeo Lozano, Santa Marta. 100 p.

López-Pérez, A. 2017. Revisiting the Cenozoic history and the origin of the Eastern Pacific coral fauna. 30-57. In: Glynn, P.W., D.P. Manzanello, and I.C. Enochs. (Eds.). Coral reefs of the Eastern Tropical Pacific. Springer, Dordrecht. https://doi.org/10.1007/978-94-017-7499-4_2

Maldonado, M., M.C. Carmona, R.W.M. Van Soest, and S.A. Pomponi. 2001. First record of the sponge genera Crambe and Discorhabdella for the Eastern Pacific, with description of three new species. J. Nat. Hist., 35(9): 1261-1276. https://doi.org/10.1080/002229301750384293

Marshall, W. 1876. Ideen über die Verwandtschaftsverhältnisse der Hexactinelliden. Z. wiss. Zool., 27(1): 113-136.

Merejkowsky, C. 1879. Études sur les Éponges de la Mer Blanche. Mem. Acad. Sci. St. Petersb., 26(7): 1-51.

Miloslavich, P., J.M. Díaz, E. Klein, J.J. Alvarado, C. Díaz, J. Gobin, E. Escobar-Briones, J.J. Cruz-Motta, E. Weil, J. Cortés, A.C. Bastidas, R. Robertson, F. Zapata, A. Martín, J. Castillo, A. Kazandjian, and M. Ortiz. 2010. Marine biodiversity in the Caribbean: regional estimates and distribution patterns. PloS ONE, 5(8): e11916. https://doi.org/10.1371/journal.pone.0011916

Minchin, E.A. 1900. Sponges: 1-178. In: Lankester, E. R. (Ed.). A treatise on zoology. The Porifera and Coelenterata. Adam Charles Black, London.

Morrow, C. and P. Cárdenas. 2015. Proposal for a revised classification of the Demospongiae (Porifera). Front. Zool., 12: 7. https://doi.org/10.1186/s12983015-0099-8

Morrow, C., B. Picton, D. Erpenbeck, N. Boury-Esnault, C. Maggs and A. Allcock. 2012. Congruence between nuclear and mitochondrial genes in Demospongiae: A new hypothesis for relationships within the G4 clade (Porifera: Demospongiae). Mol. Phylog. Evol., 62(1): 174-190. https://doi. org/10.1016/j.ympev.2011.09.016

Narváez, K. 1999. Identificación y aspectos ecológicos de las esponjas del arrecife coralino de Playa Blanca, isla Gorgona (Pacífico colombiano). Tesis Biol. Mar., Univ. Valle, Cali. 57 p.

Nichols, A. 2005. An evaluation of support for order-level monophyly and interrelationships within the class Demospongiae using partial data from the large subunit rDNA and cytochrome oxidase subunit I. Mol. Phylog. Evol., 34: 81-96. https://doi.org/10.1016/j.ympev.2004.08.019

Nichols, A. and A.G. Barnes. 2005. A molecular phylogeny and historical biogeography of the marine sponge genus Placospongia (Phylum Porifera) indicate low dispersal capabilities and widespread crypsis. J. Exp. Mar. Biol. Ecol., 323: 1-15. https://doi.org/10.1016/j.jembe.2005.02.012

Posada, B.O., W. Henao y G. Guzmán. 2009. Diagnóstico de la erosión y sedimentación en la zona costera del Pacífico colombiano. Ser. Publ. Espec., (17). Invemar, Santa Marta. 148 p.

Ridley, S.O. and A. Dendy. 1887. Report on the Monaxonida collected by H.M.S. 'Challenger' during the years 1873-1876. Report on the scientific results of the voyage of H.M.S. 'Challenger', 1873-1876. Zoology, 20(59): 1-275.

Row, R.W.H. 1911. Reports on the marine biology of the Sudanese Red Sea, from Collections made by Cyril Crossland, M. A., B.Sc., F.Z.S. XIX. Report on the Sponges collected by Mr. Cyril Crossland in 1904-5. Part II. Non-Calcarea. Zoology, 31(208): 287-400. https://doi.org/10.1111/j.1096.-3642.1911. tb00461.x 
Rützler, K. and K.P. Smith. 1993. The genus Terpios (Suberitidae) and new species in the "Lobiceps" complex. Sci. Mar., 57(4): $381-393$.

Rützler, K., C. Piantoni, R.W.M. Van Soest, and M.C. Díaz. 2014. Diversity of sponges (Porífera) from cryptic habitats on the Belize barrier reef near Carrie Bow Cay. Zootaxa, 3805(1): 1-129. https://doi.org/10.11646/zootaza.3805.1.1

Schmidt, O. 1862. Die Spongien des adriatischen Meeres. Wilhelm Engelmann, Leipzig. 88 p.

Schmidt, O. 1870. Grundzüge einer Spongien-Fauna des atlantischen Gebietes. Wilhelm Engelmann, Leipzig. 88 p.

Schulze, F.E. 1877. Untersuchungen über den Bau und die Entwicklung der Spongien. Die Gattung Halisarca. Z. wiss. Zool., $28:$ 1-48.

Schulze, F.E. 1878. Untersuchungen über den Bau und die Entwicklung der Spongien. Vierte Mittheilung. Die Familie der Aplysinidae. Z. wiss. Zool., 30: $379-420$.

Schuster, A, D. Erpenbeck, A. Pisera, J.N.A. Hooper, M. Bryce, and J. Fromont. 2015. Deceptive desmas: molecular phylogenetics suggests a new classification and uncovers convergent evolution of lithistid demosponges. PLoS ONE 10(1): e116038. https://doi.org/10.1371/journal.pone.0116038

Silva, J.A. and S. Zea. 2017. New records of sponges of the genera Petrosia and Xestospongia (Demospongiae: Haplosclerida: Petrosiidae) from the Colombian Caribbean. Bol. Investig. Mar. Costeras, 46(1): 114-134.

Sollas, W.J. 1885. A classification of the sponges. Ann. Mag. Nat. Hist., 16(95): 1-395. https://doi.org/10.1080/00222938509459901

Sollas, W.J. 1887. Sponges: 412-429. In: Black, A. (Ed.), Encyclopaedia Britannica. $9^{\text {th }}$ edition, 22.

Sollas, W.J. 1888. Report on the Tetractinellida collected by H.M.S. Challenger, during the years 1873-1876. Report on the Scientific Results of the Voyage of H.M.S. Challenger, 1873-1876. Zoology, 25(63): 273-274. https://doi.org/10.5962/bhl.title.6513

Thiele, J. 1905. Die kiesel-und hornschwämme der Sammlug plate. Zoologische Jahrbücher, Supplementun 6 (Fauna Chilensis III): 417-490.

Topsent, E. 1889. Quelques spongiaires du Banc de Campêche et de la Pointe-à-Pître. Mém. Soc. Zool. France, 2: 30-52.

Topsent, E. 1893. Nouvelle série de diagnoses d'éponges de Roscoff et de Banyuls. Arch. Zool. Exp. Gén., 10: 33-43.

Topsent, E. 1928. Spongiaires de l'Atlantique et de la Méditerranée provenant des croisières du Prince Albert ler de Monaco. Résultats des campagnes scientifiques accomplies par le Prince Albert I, Monaco, 74: 1-376.

Van Soest, R.W.M. 1994. Demospongiae distribution patterns. 213-223. En: Soest, R.W.M. van, T.M.G. Kempenn van, and J.C. Braekman. (Eds.) Sponges in time and space: biology, chemistry, paleontology. Balkema, Amsterdam. 515 p.

Van Soest, R.W.M. 2009. New sciophilous sponges from the Caribbean (Porifera: Demospongiae). Zootaxa, 2107: 1-40. https://doi.org/10.11646/ zootaxa.2107.1.1

Van Soest, R.W.M. 2017. Sponges of the Guyana shelf. Zootaxa, 4217: 1-225. https://doi.org/10.11646/zootaxa.4217.1.1

Van Soest, R.W.M. and E. Hajdu. 2000. New species of Desmanthus (Porifera, Demospongiae) with a discussion of its ordinal relationships. Zoosystema, 22(2): 299-312.

Van Soest, R.W.M., M.C. Díaz, and S.A. Pomponi. 1990. Phylogenetic classification of the Halichondrids (Porifera, Demospongiae). Beaufortia, 40(2): $15-62$.

Van Soest, R.W.M., D. Erpenbeck, and B. Álvarez. 2002. Family Dictyonellidae Van Soest, Díaz and Pomponi, 1990. 773-786. In: Hooper, J.N.A., R.W.M. Van Soest, and P. Willenz (Eds.). Systema Porifera. A guide to the classification of sponges. Kluwer Academic/Plenum Publishers: New York. 1100 p. https://doi.org/10.1007/978-1-4615-0747-5_83

Van Soest, R.W.M., N. Boury-Esnault, J. Vacelet, M. Dohrmann, D. Erpenbeck, N.J. De Voogd, N. Santodomingo, B. Vanhoorne, M. Kelly, and J.N.A. Hooper. 2012. Global diversity of sponges (Porifera). PLoS ONE, 7(4): e35105. doi:10.1371/journal.pone.0035105

Van Soest, R.W.M., N. Boury-Esnault, J.N.A. Hooper, K. Rützler, N.J. de Voogd, B. Álvarez, E. Hajdu, A.B. Pisera, R. Manconi, C. Schönberg, M. Klautau, B. Picton, M. Kelly, J. Vacelet, M. Dohrmann, M.C. Díaz, P. Cárdenas, J.L. Carballo, P. Ríos, and R. Downey. 2018. World Porifera database. http:// www.marinespecies.org/porifera 14/09/2018.

Vega, C. 2012. Composición y afinidades biogeográficas de esponjas (Demospongiae) asociadas a comunidades coralinas del Pacífico mexicano. Tesis Ph.D. Ciencias Mar. Inst. Politéc. Nal. La Paz, México. 253 p.

Velandia, M.C. y J.M Díaz. 2016. Atlas marino-costero del Pacífico norte colombiano. Fundación Marviva, Bogotá. 130 p.

Veron, J.E.N. 1995. Corals in space and time. The biogeography and evolution of the Scleractinia. xiii +. UNSW press, Sydney. 321 p. https://doi.org/10.1017/ S0016756800008050

Vosmaer, G.C.J. and J.H. Vernhout. 1902. The Porifera of the Siboga Expedition I. The Genus Placospongia. In: Siboga Expedites, 6(a): 1-17. 
Wang, C., C. Deser, J.Y. Yu, P. DiNezio, and A. Clement. 2017. El Niño and Southern Oscillation (ENSO): A review. 84-106. In: Glynn, P.W., D.P. Manzanello, and I.C. Enochs. (Eds.). Coral reefs of the Eastern Tropical Pacific. Springer: Dordrecht. https://hdl.handle.net/10.1007/978-94-017$7499-4$

Wiedenmayer, F. 1977. Shallow-water sponges of the western Bahamas. Experientia Suppl., 28: 1-287. https://doi.org/10.1007/978-3-0348-5797-0

Worheide, G., A. Cava, and J.N.A. Hooper. 2005. Biodiversity, molecular ecology and phylogeography of marine sponges: patterns, implications and outlooks. Integr. Comp. Biol., 45: 377-385. https://doi.org/10.1093/icb/45.2.377

Wulff, J.L. 1996. Do the same sponge species live on both the Caribbean and Eastern Pacific sides of the Isthmus of Panama? Bull. Inst. Royal Sci. Nat. Belgique, 66: 165-173.

Wulff, J.L. 1997. Causes and consequences of differences in sponges diversity and abundance between the Caribbean and Eastern Pacific at Panamá. 13771382. In: H.A. Lessios and I.G. Macintyre. (Eds.). Proc. $8^{\text {th }}$ Internat. Coral Reef Symp. Vol. 2. Smithsonian Tropical Research Institute, Panama.

Zea, S. 1987. Esponjas del Caribe colombiano. Catalogo Científico, Bogotá. 286 p.

Zea, S. 1998. Estado actual del conocimiento de sistemática de esponjas marinas (Porifera) del Caribe colombiano. Bol. Ecotrópica, 33: 45-59.

Zea, S., T.P. Henkel, and J.R. Pawlik. 2014. The sponge guide: a picture guide to Caribbean sponges. $3^{\text {rd }}$ edition. www.spongeguide.org 08/05/2018.

RECIBIDO/RECEIVED: 26/06/2019

ACEPTADO/ACCEPTED: 11/09/2020 